\title{
Large deviation principles for first-order scalar conservation laws with stochastic forcing
}

\author{
Zhao Dong ${ }^{1,2}$, Jiang-Lun $\mathrm{Wu}^{3}$, Rangrang Zhang ${ }^{4, *}$, Tusheng Zhang ${ }^{5}$ \\ ${ }^{1}$ RCSDS, Academy of Mathematics and Systems Science, Chinese Academy of Sciences, Beijing 100190, China \\ ${ }^{2}$ School of Mathematical Sciences, University of Chinese Academy of Sciences, Beijing 100049, China \\ ${ }^{3}$ Department of Mathematics, Swansea University, Bay Campus, Swansea SA1 8EN, UK \\ ${ }^{4}$ School of Mathematics and Statistics, Beijing Institute of Technology, Beijing 100081, China \\ ${ }^{5}$ School of Mathematics, University of Manchester, Oxford Road, Manchester M13 9PL, England, UK \\ (dzhao@amt.ac.cn, j.l.wu@swansea.ac.uk, rrzhang@amss.ac.cn, tusheng.zhang@manchester.ac.uk)
}

\begin{abstract}
In this paper, we established the Freidlin-Wentzell type large deviation principles for firstorder scalar conservation laws perturbed by small multiplicative noise. Due to the lack of the viscous terms in the stochastic equations, the kinetic solution to the Cauchy problem for these first-order conservation laws is studied. Then, based on the well-posedness of the kinetic solutions, we show that the large deviations holds by utilising the weak convergence approach.
\end{abstract}

AMS Subject Classification: Primary 60F10; Secondary 60H15.

Keywords: large deviations; first-order conservation laws; weak convergence approach; kinetic solution.

\section{Introduction}

This paper concerns the asymptotic behaviour of stochastic scalar conservation laws with small multiplicative noise. The (deterministic) conservation laws (in both scalar and vectorial) are fundamental to our understanding of the space-time evolution laws of interesting physical quantities, in that they describe (dynamical) processes that can or cannot occur in nature. Mathematically or statistically, such physical laws should incorporate with noise influences, due to the lack of knowledge of certain physical parameters as well as bias or incomplete measurements arising in experiments or modeling. More precisely, fix any $T>0$ and let $\left(\Omega, \mathcal{F}, P,\left\{\mathcal{F}_{t}\right\}_{t \in[0, T]},\left(\left\{\beta_{k}(t)\right\}_{t \in[0, T]}\right)_{k \in \mathbb{N}}\right)$ be a stochastic basis. Without loss of generality, here the filtration $\left\{\mathcal{F}_{t}\right\}_{t \in[0, T]}$ is assumed to be complete and $\left\{\beta_{k}(t)\right\}_{t \in[0, T]}, k \in \mathbb{N}$, are independent (one-dimensional) $\left\{\mathcal{F}_{t}\right\}_{t \in[0, T]}-$ Wiener processes. We use $\mathbb{E}$ to denote the expectation with respect to $P$. Fix any $N \in \mathbb{N}$, let $\mathbb{T}^{N} \subset \mathbb{R}^{N}$ denote the $N$-dimensional torus. We are concerned with the following scalar conservation law with stochastic forcing

$$
d u+\operatorname{div}(A(u)) d t=\Phi(u) d W(t) \quad \text { in } \mathbb{T}^{N} \times[0, T]
$$

${ }^{*}$ Corresponding author. 
for a random field $u:(\omega, x, t) \in \Omega \times \mathbb{T}^{N} \times[0, T] \mapsto u(\omega, x, t)=: u(x, t) \in \mathbb{R}$, that is, the equation is periodic in the space variable $x \in \mathbb{T}^{N}$, where the flux function $A: \mathbb{R} \rightarrow \mathbb{R}^{N}$ and the coefficient $\Phi: \mathbb{R} \rightarrow \mathbb{R}$ are measurable and fulfill certain conditions specified later, and $W$ is a cylindrical Wiener process defined on a given (separable) Hilbert space $U$ with the form $W(t)=\sum_{k \geq 1} \beta_{k}(t) e_{k}, t \in[0, T]$, where $\left(e_{k}\right)_{k \geq 1}$ is a complete orthonormal base in the Hilbert space $U$. We consider the following Cauchy problem

$$
\left\{\begin{array}{l}
d u+\operatorname{div}(A(u)) d t=\Phi(u) d W(t) \quad \text { in } \mathbb{T}^{N} \times(0, T], \\
u(\cdot, 0)=u_{0}(\cdot) \quad \text { on } \mathbb{T}^{N} .
\end{array}\right.
$$

For the deterministic case, i.e., $\Phi \equiv 0,(1.1)$ is well studied in the PDEs literature, see e.g. the monograph [6] and the most recent reference Ammar, Willbold and Carrillo [1] (and references therein). As well known, the Cauchy problem for the deterministic first-order PDE (1.1) does not admit any (global) smooth solutions, but there exist infinitely many weak solutions to the deterministic Cauchy problem and an additional entropy condition has to be added to get the uniqueness and further to identify the physical weak solution. The notion of entropy solutions for the deterministic problem in the $L^{\infty}$ framework was initiated by Otto in [23]. Moreover, Porretta and Vovelle [24] studied the problem in the $L^{1}$ setting, that is, the solutions are allowed to be unbounded. In order to deal with unbounded solutions, they defined a notion of renormalized entropy solutions which generalizes Otto's original definition of entropy solutions. The kinetic formulation of weak entropy solution of the Cauchy problem for a general multidimensional scalar conservation law, named as the kinetic system, is derived by Lions, Perthame and Tadmor in [19]. They further discussed the relationship between entropy solutions and the kinetic system.

Having a stochastic forcing term in (1.1) is very natural and important for various modeling problems arising in a wide variety of fields, e.g., physics, engineering, biology and so on. The Cauchy problem for the stochastic equation (1.1) driven by additive noise has been studied by Kim in [17] wherein the author proposed a method of compensated compactness to prove the existence of a stochastic weak entropy solution via vanishing viscosity approximation. Moreover, a Kruzkov-type method was used there to prove the uniqueness. Furthermore, Vallet and Wittbold [25] extended the results of Kim to the multidimensional Dirichlet problem with additive noise. By utilising the vanishing viscosity method, Young measure techniques, and Kruzkov doubling variables technique, they managed to show the existence and uniqueness of the stochastic entropy solutions. Concerning the case of the equation with multiplicative noise, for Cauchy problem over the whole spatial space, Feng and Nualart [13] introduced a notion of strong entropy solutions in order to prove the uniqueness of the entropy solution. Using the vanishing viscosity and compensated compactness arguments, they established the existence of stochastic strong entropy solutions only in the one-dimensional space case. On the other hand, using a kinetic formulation, Debussche and Vovelle [9] solved the Cauchy problem for (1.1) in any dimension. They made use of a notion of kinetic solutions developed by Lions, Perthame and Tadmor for deterministic, first-order scalar conservation laws in [19]. In view of the equivalence between kinetic formulation and entropy solution, they obtained the existence and uniqueness of the entropy solutions. The long-time behavior 
of periodic scalar first-order conservation laws with additive stochastic forcing under an hypothesis of non-degeneracy of the flux function is studied by Debussche and Vovelle in [10]. For sub-cubic fluxes, they show the existence of an invariant measure. Moreover, for sub-quadratic fluxes, they prove the uniqueness and ergodicity of the invariant measure.

From statistical mechanics point of view, asymptotic analysis for vanishing the noise force is important and interesting for studying stochastic conservation laws, in which establishing large deviation principles is a core step for finer analysis as well as gaining deeper insight for the described physical evolutions. Due to lack of second order elliptic operators for the space variable, the asymptotic analysis for stochastic conservation laws is really challenging and all those existing approaches for establishing large deviation principles seem unapplicable. To our knowledge, Mariani [20] (see also [21] for more details) is the first work towards large deviations for stochastic conservation laws, wherein the author considered a family of stochastic conservation laws as parabolic SPDEs with additional small viscosity term and small (spatially) regularized (i.e., spatially smoothing) noises. By a very interesting scaling procedure and deep insightful observations from interacting particle systems, Mariani has succeeded to establish large deviation principles by vanishing viscosity and noise terms simultaneously in a smart choice of scalings. While, large deviations for the stochastic first-order conservation laws remain open. Due to the fact that the entropy solutions are living in rather irregular spaces comparing to various type solutions for parabolic SPDEs, it is indeed a challenge to establish large deviation principles for the first-order conservation laws with general noise force.

The purpose of this paper is to prove the Freidlin-Wentzell type large deviation principle (LDP) for the first-order stochastic scalar conservation law in $L^{1}\left([0, T] ; L^{1}\left(\mathbb{T}^{N}\right)\right)$, which provides the exponential decay of small probabilities associated with the corresponding stochastic dynamical systems with small noise. An important tool for studying the Freidlin-Wentzell's LDP is the weak convergence approach, which is developed by Dupuis and Ellis in [12]. The key idea of this approach is to prove certain variational representation formula about the Laplace transform of bounded continuous functionals, which then leads to the verification of the equivalence between the LDP and the Laplace principle. In particular, for Brownian functionals, an elegant variational representation formula has been established by Boué and Dupuis in [2] and by Budhiraja and Dupuis in [3]. Recently, a sufficient condition to verify the large deviation criteria of Budhiraja, Dupuis and Maroulas for functionals of Brownian motions is proposed by Matoussi, Sabbagh and Zhang in [22], which turns out to be more suitable for SPDEs arising from fluid mechanics. Thus, in the present paper, we adopt this new sufficient condition.

Our proof strategy mainly consists of the following procedures. As an important part of the proof, we need to obtain the global well-posedness of the associated skeleton equations. For showing the uniqueness, we apply the doubling of variables method. For showing the existence result, we first apply the vanishing viscosity method to construct a sequence of approximating equations as in [9]. Then, we prove that the family of the solutions of the approximating equations is compact in an appropriate space and that any limit of the approximating solutions gives rise to a solution of the associated skeleton equation. To complete the proof of the large deviation principle, we also need to study the weak convergence 
of the small noise perturbations of the problem (1.1) in the random directions of the Cameron-Martin space of the driving Brownian motions. To verify the convergence of the randomly perturbed equation to the corresponding unperturbed equation in $L^{1}\left([0, T] ; L^{1}\left(\mathbb{T}^{N}\right)\right)$, the doubling of variables method plays a key role.

The rest of the paper is organised as follows. The mathematical formulation of stochastic scalar conservation laws is presented in Section 2. In Section 3, we introduce the weak convergence method and state our main result. Section 4 is devoted to the study of the associated skeleton equations. The large deviation principle is proved in Section 5.

\section{Preliminaries}

Let $\mathcal{L}\left(K_{1}, K_{2}\right)$ (resp. $\left.\mathcal{L}_{2}\left(K_{1}, K_{2}\right)\right)$ be the space of bounded (resp. Hilbert-Schmidt) linear operators from a Hilbert space $K_{1}$ to another Hilbert space $K_{2}$, whose norm is denoted by $\|\cdot\|_{\mathcal{L}\left(K_{1}, K_{2}\right)}$ (resp. $\|$. \|\|$\left._{\mathcal{L}_{2}\left(K_{1}, K_{2}\right)}\right)$. Further, $C_{b}$ represents the space of bounded, continuous functions and $C_{b}^{1}$ stands for the space of bounded, continuously differentiable functions having bounded first order derivative. Let $\|\cdot\|_{L^{p}}$ denote the norm of Lebesgue space $L^{p}\left(\mathbb{T}^{N}\right)$ for $p \in(0, \infty]$. In particular, set $H=L^{2}\left(\mathbb{T}^{N}\right)$ with the corresponding norm $\|\cdot\|_{H}$. For all $a \geq 0$, let $H^{a}\left(\mathbb{T}^{N}\right)=W^{a, 2}\left(\mathbb{T}^{N}\right)$ be the usual Sobolev space of order $a$ with the norm

$$
\|u\|_{H^{a}}^{2}=\sum_{|\alpha|=\left|\left(\alpha_{1}, \ldots, \alpha_{N}\right)\right|=\alpha_{1}+\cdots+\alpha_{N} \leq a} \int_{\mathbb{T}^{N}}\left|D^{\alpha} u(x)\right|^{2} d x .
$$

$H^{-a}\left(\mathbb{T}^{N}\right)$ stands for the topological dual of $H^{a}\left(\mathbb{T}^{N}\right)$, whose norm is denoted by $\|\cdot\|_{H^{-a}}$. Moreover, we use the brackets $\langle\cdot, \cdot\rangle$ to denote the duality between $C_{c}^{\infty}\left(\mathbb{T}^{N} \times \mathbb{R}\right)$ and the space of distributions over $\mathbb{T}^{N} \times \mathbb{R}$. Similarly, for $1 \leq p \leq \infty$ and $q:=\frac{p}{p-1}$, the conjugate exponent of $p$, we denote

$$
\langle F, G\rangle:=\int_{\mathbb{T}^{N}} \int_{\mathbb{R}} F(x, \xi) G(x, \xi) d x d \xi, \quad F \in L^{p}\left(\mathbb{T}^{N} \times \mathbb{R}\right), G \in L^{q}\left(\mathbb{T}^{N} \times \mathbb{R}\right),
$$

and also for a measure $m$ on the Borel measurable space $\mathbb{T}^{N} \times[0, T] \times \mathbb{R}$

$$
m(\phi):=\langle m, \phi\rangle:=\int_{\mathbb{T}^{N} \times[0, T] \times \mathbb{R}} \phi(x, t, \xi) d m(x, t, \xi), \quad \phi \in C_{b}\left(\mathbb{T}^{N} \times[0, T] \times \mathbb{R}\right) .
$$

\subsection{Kinetic solution and generalized kinetic solution}

Let us recall the notion of a solution to equation $(1.1)$ from $[9,10]$. Keeping in mind that we are working on the stochastic basis $\left(\Omega, \mathcal{F}, P,\left\{\mathcal{F}_{t}\right\}_{t \in[0, T]},\left(\beta_{k}(t)\right)_{k \in \mathbb{N}}\right)$.

Definition 2.1. (Kinetic measure) A map $m$ from $\Omega$ to the set of non-negative, finite measures over $\mathbb{T}^{N} \times[0, T] \times \mathbb{R}$ is said to be a kinetic measure, if

1. $m$ is measurable, that is, for each $\phi \in C_{b}\left(\mathbb{T}^{N} \times[0, T] \times \mathbb{R}\right),\langle m, \phi\rangle: \Omega \rightarrow \mathbb{R}$ is measurable, 
2. $m$ vanishes for large $\xi$, i.e.,

$$
\lim _{R \rightarrow+\infty} \mathbb{E}\left[m\left(\mathbb{T}^{N} \times[0, T] \times B_{R}^{c}\right)\right]=0
$$

where $B_{R}^{c}:=\{\xi \in \mathbb{R},|\xi| \geq R\}$

3. for every $\phi \in C_{b}\left(\mathbb{T}^{N} \times \mathbb{R}\right)$, the process

$$
(\omega, t) \in \Omega \times[0, T] \mapsto \int_{\mathbb{T}^{N} \times[0, t] \times \mathbb{R}} \phi(x, \xi) d m(x, s, \xi) \in \mathbb{R}
$$

is predictable.

Let $\mathcal{M}_{0}^{+}\left(\mathbb{T}^{N} \times[0, T] \times \mathbb{R}\right)$ be the space of all bounded, nonnegative random measures $m$ satisfying (2.2).

Definition 2.2. (Kinetic solution) Let $u_{0} \in L^{\infty}\left(\mathbb{T}^{N}\right)$. A measurable function $u: \mathbb{T}^{N} \times[0, T] \times \Omega \rightarrow \mathbb{R}$ is called a kinetic solution to (1.1) with initial datum $u_{0}$, if

1. $(u(t))_{t \in[0, T]}$ is predictable,

2. for any $p \geq 1$, there exists $C_{p} \geq 0$ such that

$$
\mathbb{E}\left(\text { ess } \sup _{t \in[0, T]}\|u(t)\|_{L^{p}\left(\mathbb{T}^{N}\right)}^{p}\right) \leq C_{p}
$$

3. there exists a kinetic measure $m$ such that $f:=I_{u>\xi}$ satisfies the following

$$
\begin{aligned}
& \int_{0}^{T}\left\langle f(t), \partial_{t} \varphi(t)\right\rangle d t+\left\langle f_{0}, \varphi(0)\right\rangle+\int_{0}^{T}\langle f(t), a(\xi) \cdot \nabla \varphi(t)\rangle d t \\
= & -\sum_{k \geq 1} \int_{0}^{T} \int_{\mathbb{T}^{N}} g_{k}(x) \varphi(x, t, u(x, t)) d x d \beta_{k}(t) \\
& -\frac{1}{2} \sum_{k \geq 1} \int_{0}^{T} \int_{\mathbb{T}^{N}} \partial_{\xi} \varphi(x, t, u(x, t)) G^{2}(x) d x d t+m\left(\partial_{\xi} \varphi\right), \text { a.s. },
\end{aligned}
$$

for all $\varphi \in C_{c}^{1}\left(\mathbb{T}^{N} \times[0, T] \times \mathbb{R}\right)$, where $u(t)=u(\cdot, t, \cdot), G^{2}=\sum_{k=1}^{\infty}\left|g_{k}\right|^{2}$ and $a(\xi):=A^{\prime}(\xi)$.

In order to prove the existence of a kinetic solution, the generalized kinetic solution was introduced in [9].

Definition 2.3. (Young measure) Let $(X, \lambda)$ be a finite measure space. Let $\mathcal{P}_{1}(\mathbb{R})$ denote the set of all (Borel) probability measures on $\mathbb{R}$. A map $v: X \rightarrow \mathcal{P}_{1}(\mathbb{R})$ is said to be a Young measure on $X$, iffor each $\phi \in C_{b}(\mathbb{R})$, the map $z \in X \mapsto v_{z}(\phi) \in \mathbb{R}$ is measurable. Next, we say that a Young measure $v$ vanishes at infinity if, for each $p \geq 1$, the following holds

$$
\int_{X} \int_{\mathbb{R}}|\xi|^{p} d v_{z}(\xi) d \lambda(z)<+\infty .
$$


Definition 2.4. (Kinetic function) Let $(X, \lambda)$ be a finite measure space. A measurable function $f: X \times \mathbb{R} \rightarrow$ $[0,1]$ is called a kinetic function, if there exists a Young measure $v$ on $X$ that vanishes at infinity such that $\forall \xi \in \mathbb{R}$

$$
f(z, \xi)=v_{z}(\xi,+\infty)
$$

holds for $\lambda-$ a.e. $z \in X$. We say that $f$ is an equilibrium if there exists a measurable function $u: X \rightarrow \mathbb{R}$ such that $f(z, \xi)=I_{u(z)>\xi}$ a.e., or equivalently, $v_{z}=\delta_{u(z)}$ for $\lambda-$ a.e. $z \in X$.

Let $f: X \times \mathbb{R} \rightarrow[0,1]$ be a kinetic function, we use $\bar{f}$ to denote its conjugate function $\bar{f}:=1-f$.

Definition 2.5. (Generalized kinetic solution) Let $f_{0}: \Omega \times \mathbb{T}^{N} \times \mathbb{R} \rightarrow[0,1]$ be a kinetic function with $(X, \lambda)=\left(\Omega \times \mathbb{T}^{N}, P \otimes d x\right)$. A measurable function $f: \Omega \times \mathbb{T}^{N} \times[0, T] \times \mathbb{R} \rightarrow[0,1]$ is said to be a generalized kinetic solution to (1.1) with initial datum $f_{0}$, if

1. $(f(t))_{t \in[0, T]}$ is predictable,

2. $f$ is a kinetic function with $(X, \lambda)=\left(\Omega \times \mathbb{T}^{N} \times[0, T], P \otimes d x \otimes d t\right)$ and for any $p \geq 1$, there exists a constant $C_{p}>0$ such that $v:=-\partial_{\xi}$ f fulfills the following

$$
\mathbb{E}\left(\text { ess } \sup _{t \in[0, T]} \int_{\mathbb{T}^{N}} \int_{\mathbb{R}}|\xi|^{p} d v_{x, t}(\xi) d x\right) \leq C_{p},
$$

3. there exists a kinetic measure $m$ such that for $\varphi \in C_{c}^{1}\left(\mathbb{T}^{N} \times[0, T] \times \mathbb{R}\right)$,

$$
\begin{aligned}
& \int_{0}^{T}\left\langle f(t), \partial_{t} \varphi(t)\right\rangle d t+\left\langle f_{0}, \varphi(0)\right\rangle+\int_{0}^{T}\langle f(t), a(\xi) \cdot \nabla \varphi(t)\rangle d t \\
= & -\sum_{k \geq 1} \int_{0}^{T} \int_{\mathbb{T}^{N}} \int_{\mathbb{R}} g_{k}(x) \varphi(x, t, \xi) d v_{x, t}(\xi) d x d \beta_{k}(t) \\
& -\frac{1}{2} \int_{0}^{T} \int_{\mathbb{T}^{N}} \int_{\mathbb{R}} \partial_{\xi} \varphi(x, t, \xi) G^{2}(x) d v_{x, t}(\xi) d x d t+m\left(\partial_{\xi} \varphi\right), \text { a.s.. }
\end{aligned}
$$

Referring to [9], almost surely, any generalized solution admits possibly different left and right weak limits at any point $t \in[0, T]$. This property is important for establishing a comparison principle which allows to prove uniqueness. The following result is proved in [9].

Proposition 2.1. (Left and right weak limits) Let $f_{0}$ be a kinetic initial datum and $f$ be a generalized kinetic solution to (1.1) with initial $f_{0}$. Then $f$ admits, almost surely, left and right limits respectively at every point $t \in[0, T]$. More precisely, for any $t \in[0, T]$, there exist kinetic functions $f^{t \pm}$ on $\Omega \times \mathbb{T}^{N} \times \mathbb{R}$ such that $\mathbb{P}$-a.s.

$$
\langle f(t-\varepsilon), \varphi\rangle \rightarrow\left\langle f^{t-}, \varphi\right\rangle
$$

and

$$
\langle f(t+\varepsilon), \varphi\rangle \rightarrow\left\langle f^{t+}, \varphi\right\rangle
$$


as $\varepsilon \rightarrow 0$ for all $\varphi \in C_{c}^{1}\left(\mathbb{T}^{N} \times \mathbb{R}\right)$. Moreover, almost surely,

$$
\left\langle f^{t+}-f^{t-}, \varphi\right\rangle=-\int_{\mathbb{T}^{N} \times[0, T] \times \mathbb{R}} \partial_{\xi} \varphi(x, \xi) I_{\{t\}}(s) d m(x, s, \xi) .
$$

In particular, almost surely, the set of $t \in[0, T]$ fulfilling that $f^{t+} \neq f^{t-}$ is countable.

For a generalized kinetic solution $f$, define $f^{ \pm}$by $f^{ \pm}(t)=f^{t \pm}, t \in[0, T]$. Since we are dealing with the filtration associated to Brownian motion, both $f^{ \pm}$are clearly predictable as well. Also $f=f^{+}=f^{-}$ almost everywhere in time and we can take any of them in an integral with respect to the Lebesgue measure or in a stochastic integral. However, if the integral is with respect to a measure-typically a kinetic measure in this article, the integral is not well defined for $f$ and may differ if one chooses either $f^{+}$or $f^{-}$.

Finally in this subsection, as a special example, let us consider the following stochastic heat equation on $\mathbb{T}^{N} \times[0, \infty)$

$$
d u-\Delta u d t=\Phi(u) d W(t), \quad u(x, 0)=u_{0}(x) .
$$

We aim to derive an explicit expression of its kinetic measure $m$. For this, we have the following kinetic formulation

Proposition 2.2. Let $u_{0} \in L^{\infty}\left(\mathbb{T}^{N}\right)$ and $u$ be the solution to (2.7). Then $f:=I_{u>\xi}$ satisfies the following

$$
\begin{aligned}
& \int_{0}^{T}\left\langle f(t), \partial_{t} \varphi(t)\right\rangle d t+\left\langle f_{0}, \varphi(0)\right\rangle-\int_{0}^{T}\langle f(t), \Delta \varphi(t)\rangle d t \\
= & -\sum_{k \geq 1} \int_{0}^{T} \int_{\mathbb{T}^{N}} \int_{\mathbb{R}} g_{k}(x, \xi) \varphi(x, t, \xi) d v_{x, t}(\xi) d x d \beta_{k}(t) \\
& -\frac{1}{2} \int_{0}^{T} \int_{\mathbb{T}^{N}} \int_{\mathbb{R}} \partial_{\xi} \varphi(x, t, \xi) G^{2}(x, \xi) d v_{x, t}(\xi) d x d t+m\left(\partial_{\xi} \varphi\right), \text { a.s. }
\end{aligned}
$$

for all $\varphi \in C_{c}^{1}\left(\mathbb{T}^{N} \times[0, T) \times \mathbb{R}\right)$, where $f_{0}(\xi)=I_{u_{0}>\xi}$ and for all $\phi \in C_{b}\left(\mathbb{T}^{N} \times[0, T] \times \mathbb{R}\right)$,

$$
d v_{x, t}(\xi)=\delta_{u=\xi} d \xi, \quad m(\phi)=\int_{0}^{T} \int_{\mathbb{T}^{N}} \phi(x, t, u(x, t))|\nabla u|^{2} d x d t
$$

Proof. By Itô formula, we have for $\theta \in C^{2}(\mathbb{R})$ with polynomial growth at $\pm \infty$,

$$
\begin{aligned}
d\left(I_{u>\xi}, \theta^{\prime}\right) & :=d \int_{\mathbb{R}} I_{u>\xi} \theta^{\prime}(\xi) d \xi=d \theta(u) \\
& =\theta^{\prime}(u)(\Delta u d t+\Phi(u) d W(t))+\frac{1}{2} \theta^{\prime \prime}(u) G^{2} d t,
\end{aligned}
$$

where $G^{2}=\sum_{k \geq 1}\left|g_{k}\right|^{2}$. 
The first term can be rewritten as

$$
\theta^{\prime}(u) \Delta u=\Delta \theta(u)-|\nabla u|^{2} \theta^{\prime \prime}(u)=\Delta\left(I_{u>\xi}, \theta^{\prime}\right)+\left(\partial_{\xi}\left(|\nabla u|^{2} \delta_{u=\xi}\right), \theta^{\prime}\right)
$$

Hence, we obtain the following kinetic formulation:

$$
\begin{aligned}
d\left(I_{u>\xi}, \theta^{\prime}\right)= & \Delta\left(I_{u>\xi}, \theta^{\prime}\right) d t+\left(\partial_{\xi}\left(|\nabla u|^{2} \delta_{u=\xi}-\frac{1}{2} G^{2} \delta_{u=\xi}\right), \theta^{\prime}\right) d t \\
& +\sum_{k \geq 1}\left(\delta_{u=\xi} g_{k}, \theta^{\prime}\right) d \beta_{k} .
\end{aligned}
$$

Taking $\theta(\xi)=\int_{\infty}^{\xi} \chi$, we have

$$
\begin{aligned}
d\left(I_{u>\xi}, \chi\right):= & \Delta\left(I_{u>\xi}, \chi\right) d t+\left(\partial_{\xi}\left(|\nabla u|^{2} \delta_{u=\xi}-\frac{1}{2} G^{2} \delta_{u=\xi}\right), \chi\right) d t \\
& +\sum_{k \geq 1}\left(\delta_{u=\xi} g_{k}, \chi\right) d \beta_{k} .
\end{aligned}
$$

Since the test functions $\varphi(x, \xi)=\alpha(x) \chi(\xi)$ form a dense subset of $C_{c}^{\infty}\left(\mathbb{T}^{N} \times \mathbb{R}\right)$, it follows that (2.8) holds. We complete the proof.

From above, it is clear that the kinetic measure $m$ has an explicit expression

$$
m=|\nabla u|^{2} \delta_{u=\xi}
$$

\subsection{Compactness results}

Recall the following two compactness results from [9], which are important for establishing the existence of generalized kinetic solution of (1.1).

Theorem 2.3. (Compactness of Young measures) Let $(X, \lambda)$ be a finite measure space. Let $\left(v^{n}\right)$ be a sequence of Young measures on $X$ satisfying the condition (2.4) for some $p \geq 1$, namely,

$$
\sup _{n \in \mathbb{N}} \int_{X} \int_{\mathbb{R}}|\xi|^{p} d \nu_{z}^{n}(\xi) d \lambda(z)<+\infty .
$$

Then there exists a Young measure $v$ on $X$ and a subsequence which is still denoted by $\left(v^{n}\right)$ such that, for $h \in L^{1}(X)$ and for $\phi \in C_{b}(\mathbb{R})$,

$$
\lim _{n \rightarrow \infty} \int_{X} h(z) \int_{\mathbb{R}} \phi(\xi) d v_{z}^{n}(\xi) d \lambda(z)=\int_{X} h(z) \int_{\mathbb{R}} \phi(\xi) d v_{z}(\xi) d \lambda(z) .
$$

Corollary 2.4. (Compactness of Kinetic functions) Let $(X, \lambda)$ be a finite measure space. Let $\left(f_{n}\right)$ be a sequence of kinetic functions on $X \times \mathbb{R}: f_{n}(z, \xi)=v_{z}^{n}(\xi, \infty)$, where $v^{n}, n \geq 1$, are Young measures on $X$ satisfying (2.9). Then there exists a kinetic function $f$ on $X \times \mathbb{R}$ such that $f_{n} \rightarrow f$ in $L^{\infty}(X \times \mathbb{R})-$ weak $*$, as $n \rightarrow \infty$. 


\subsection{Hypotheses and the global well-posedness of (1.1)}

For the flux function $A$ and the coefficient $\Phi$, we assume the following

Hypothesis $\mathbf{H}$ The flux function $A$ belongs to $C^{2}\left(\mathbb{R} ; \mathbb{R}^{N}\right)$ and its derivative $a$ has at most polynomial growth. That is, there exist constants $C>0, p>1$ such that

$$
|a(\xi)-a(\zeta)| \leq \Gamma(\xi, \zeta)|\xi-\zeta|, \quad \Gamma(\xi, \zeta)=C\left(1+|\xi|^{p-1}+|\zeta|^{p-1}\right) .
$$

For each $u \in \mathbb{R}$, the map $\Phi(u): U \rightarrow H$ is defined by $\Phi(u) e_{k}=g_{k}(\cdot, u)$, where each $g_{k}(\cdot, u)$ is a regular function on $\mathbb{T}^{N}$. More precisely, we assume that $g_{k} \in C\left(\mathbb{T}^{N} \times \mathbb{R}\right)$ with the following bounds

$$
\begin{aligned}
G^{2}(x, u)=\sum_{k \geq 1}\left|g_{k}(x, u)\right|^{2} & \leq D_{0}\left(1+|u|^{2}\right), \\
\sum_{k \geq 1}\left|g_{k}(x, u)-g_{k}(y, v)\right|^{2} & \leq D_{1}\left(|x-y|^{2}+|u-v|^{2}\right),
\end{aligned}
$$

for $x, y \in \mathbb{T}^{N}, u, v \in \mathbb{R}$. Since $\left\|g_{k}\right\|_{H} \leq\left\|g_{k}\right\|_{C\left(\mathbb{T}^{N}\right)}$, we deduce that $\Phi(u) \in \mathcal{L}_{2}(U, H)$, for each $u \in \mathbb{R}$. Moreover, it follows from (2.12) and (2.13) that

$$
\begin{aligned}
\|\Phi(u)\|_{\mathcal{L}_{2}(U, H)}^{2} & \leq D_{0}\left(1+\|u\|_{H}^{2}\right), \\
\|\Phi(u)-\Phi(v)\|_{\mathcal{L}_{2}(U, H)}^{2} & \leq D_{1}\|u-v\|_{H}^{2} .
\end{aligned}
$$

The following result was shown in [9].

Theorem 2.5. (Existence, Uniqueness) Let $u_{0} \in L^{\infty}\left(\mathbb{T}^{N}\right)$. Assume Hypothesis $H$ holds. Then there is a unique kinetic solution $u$ to equation (1.1) with initial datum $u_{0}$. Besides, if $f$ is a generalized kinetic solution to (1.1) with initial datum $I_{u_{0}>\xi}$, then there exist $u^{+}$and $u^{-}$, representatives of $u$ such that for all $t \in[0, T], f^{ \pm}(x, t, \xi)=I_{u^{ \pm}(x, t)>\xi}$ a.s. for a.e. $(x, t, \xi)$.

Remark 1. The kinetic solution u is a strong solution in the probabilistic sense.

\section{Freidlin-Wentzell's large deviations and statement of the main result}

We start with a brief account of notions of large deviations. Let $\left\{X^{\varepsilon}\right\}_{\varepsilon>0}$ be a family of random variables defined on a given probability space $(\Omega, \mathcal{F}, \mathbb{P})$ taking values in some Polish space $\mathcal{E}$.

Definition 3.1. (Rate function) A function $I: \mathcal{E} \rightarrow[0, \infty]$ is called a rate function if $I$ is lower semicontinuous. A rate function I is called a good rate function if the level set $\{x \in \mathcal{E}: I(x) \leq M\}$ is compact for each $M<\infty$.

Definition 3.2. (Large deviation principle) The sequence $\left\{X^{\varepsilon}\right\}$ is said to satisfy the large deviation principle with rate function I if for each Borel subset $A$ of $\mathcal{E}$

$$
-\inf _{x \in A^{o}} I(x) \leq \lim _{\varepsilon \rightarrow 0} \varepsilon \log \mathbb{P}\left(X^{\varepsilon} \in A\right) \leq \lim \sup _{\varepsilon \rightarrow 0} \varepsilon \log \mathbb{P}\left(X^{\varepsilon} \in A\right) \leq-\inf _{x \in \bar{A}} I(x),
$$

where $A^{o}$ and $\bar{A}$ denote the interior and closure of $A$ in $\mathcal{E}$, respectively. 
Suppose $W(t)$ is a cylindrical Wiener process on a Hilbert space $U$ defined on a filtered probability space $\left(\Omega, \mathcal{F},\left\{\mathcal{F}_{t}\right\}_{t \in[0, T]}, \mathbb{P}\right)$ ( that is, the paths of $W$ take values in $C([0, T] ; \mathcal{U})$, where $\mathcal{U}$ is another Hilbert space such that the embedding $U \subset \mathcal{U}$ is Hilbert-Schmidt). Now we define

$$
\begin{gathered}
\mathcal{A}:=\left\{\phi: \phi \text { is a U-valued }\left\{\mathcal{F}_{t}\right\} \text {-predictable process such that } \int_{0}^{T}|\phi(s)|_{U}^{2} d s<\infty \mathbb{P} \text {-a.s. }\right\} \\
\qquad \begin{array}{c}
S_{M}:=\left\{h \in L^{2}([0, T] ; U): \int_{0}^{T}|h(s)|_{U}^{2} d s \leq M\right\} ; \\
\mathcal{A}_{M}:=\left\{\phi \in \mathcal{A}: \phi(\omega) \in S_{M}, \mathbb{P} \text {-a.s. }\right\} .
\end{array}
\end{gathered}
$$

Here and in the sequel of this paper, we will always refer to the weak topology on the set $S_{M}$.

Suppose for each $\varepsilon>0, \mathcal{G}^{\varepsilon}: C([0, T] ; \mathcal{U}) \rightarrow \mathcal{E}$ is a measurable map and let $X^{\varepsilon}:=\mathcal{G}^{\varepsilon}(W)$. Now, we list below sufficient conditions for the large deviation principle of the sequence $X^{\varepsilon}$ as $\varepsilon \rightarrow 0$.

Condition A There exists a measurable map $\mathcal{G}^{0}: C([0, T] ; \mathcal{U}) \rightarrow \mathcal{E}$ such that the following conditions hold

(a) For every $M<\infty$, let $\left\{h^{\varepsilon}: \varepsilon>0\right\} \subset \mathcal{A}_{M}$. If $h_{\varepsilon}$ converges to $h$ as $S_{M}$-valued random elements in distribution, then $\mathcal{G}^{\varepsilon}\left(W(\cdot)+\frac{1}{\sqrt{\varepsilon}} \int_{0}^{\cdot} h^{\varepsilon}(s) d s\right)$ converges in distribution to $\mathcal{G}^{0}\left(\int_{0}^{\cdot} h(s) d s\right)$.

(b) For every $M<\infty$, the set $K_{M}=\left\{\mathcal{G}^{0}\left(\int_{0}^{n} h(s) d s\right): h \in S_{M}\right\}$ is a compact subset of $\mathcal{E}$.

The following result is due to Budhiraja et al. in [3].

Theorem 3.1. If $\left\{\mathcal{G}^{\mathcal{E}}\right\}$ satisfies condition $A$, then $X^{\mathcal{E}}$ satisfies the large deviation principle on $\mathcal{E}$ with the following good rate function I defined by

$$
I(f)=\inf _{\left\{h \in L^{2}([0, T] ; U): f=\mathcal{G}^{0}\left(\int_{0} h(s) d s\right)\right\}}\left\{\frac{1}{2} \int_{0}^{T}|h(s)|_{U}^{2} d s\right\}, \quad \forall f \in \mathcal{E} .
$$

By convention, $I(f)=\infty$, if $\left\{h \in L^{2}([0, T] ; U): f=\mathcal{G}^{0}\left(\int_{0}^{*} h(s) d s\right)\right\}=\emptyset$.

Recently, a new sufficient condition (Condition B below) to verify the assumptions in condition A (hence the large deviation principle) is proposed by Matoussi, Sabagh and Zhang in [22]. It turns out this new sufficient condition is suitable for establishing the large deviation principle for the scalar conservation laws.

Condition B There exists a measurable map $\mathcal{G}^{0}: C([0, T] ; \mathcal{U}) \rightarrow \mathcal{E}$ such that the following two items hold

(i) For every $M<+\infty$, and for any family $\left\{h^{\varepsilon} ; \varepsilon>0\right\} \subset \mathcal{A}_{M}$ and any $\delta>0$,

$$
\lim _{\varepsilon \rightarrow 0} P\left(\rho\left(Y^{\varepsilon}, Z^{\varepsilon}\right)>\delta\right)=0,
$$

where $Y^{\varepsilon}:=\mathcal{G}^{\varepsilon}\left(W(\cdot)+\frac{1}{\sqrt{\varepsilon}} \int_{0}^{\cdot} h^{\varepsilon}(s) d s\right), Z^{\varepsilon}:=\mathcal{G}^{0}\left(\int_{0}^{\cdot} h^{\varepsilon}(s) d s\right)$, and $\rho(\cdot, \cdot)$ stands for the metric in the space $\mathcal{E}$.

(ii) For every $M<+\infty$ and any family $\left\{h^{\varepsilon} ; \varepsilon>0\right\} \subset S_{M}$ that converges to some element $h$ as $\varepsilon \rightarrow 0$, $\mathcal{G}^{0}\left(\int_{0} h^{\varepsilon}(s) d s\right)$ converges to $\mathcal{G}^{0}\left(\int_{0}^{\cdot} h(s) d s\right)$ in the space $\mathcal{E}$. 


\subsection{Statement of the main result}

In this paper, we are concerned with the following stochastic conservation law driven by small multiplicative noise

$$
\left\{\begin{array}{l}
d u^{\varepsilon}+\operatorname{div}\left(A\left(u^{\varepsilon}\right)\right) d t=\sqrt{\varepsilon} \Phi\left(u^{\varepsilon}\right) d W(t), \\
u^{\varepsilon}(0)=u_{0},
\end{array}\right.
$$

for $\varepsilon>0$, where $u_{0} \in L^{\infty}\left(\mathbb{T}^{N}\right)$. Under Hypothesis $\mathrm{H}$, by Theorem 2.5, there exists a unique kinetic solution $u^{\varepsilon} \in L^{1}\left([0, T] ; L^{1}\left(\mathbb{T}^{N}\right)\right)$ a.s.. Therefore, there exists a Borel-measurable function

$$
\mathcal{G}^{\varepsilon}: C([0, T] ; \mathcal{U}) \rightarrow L^{1}\left([0, T] ; L^{1}\left(\mathbb{T}^{N}\right)\right)
$$

such that $u^{\varepsilon}(\cdot)=\mathcal{G}^{\varepsilon}(W(\cdot))$.

Let $h \in L^{2}([0, T] ; U)$, we consider the following skeleton equation

$$
\left\{\begin{array}{l}
d u_{h}+\operatorname{div}\left(A\left(u_{h}\right)\right) d t=\Phi\left(u_{h}\right) h(t) d t \\
u_{h}(0)=u_{0}
\end{array}\right.
$$

The solution $u_{h}$, whose existence will be proved in next section, defines a measurable mapping $\mathcal{G}^{0}: C([0, T] ; \mathcal{U}) \rightarrow L^{1}\left([0, T] ; L^{1}\left(\mathbb{T}^{N}\right)\right)$ so that $\mathcal{G}^{0}\left(\int_{0} h(s) d s\right):=u_{h}(\cdot)$.

We are now ready to state our main result of this paper

Theorem 3.2. Let $u_{0} \in L^{\infty}\left(\mathbb{T}^{N}\right)$. Assume Hypothesis $H$ holds. Then $u^{\varepsilon}$ satisfies the large deviation principle on $L^{1}\left([0, T] ; L^{1}\left(\mathbb{T}^{N}\right)\right)$ with the good rate function I given by (3.16).

\section{Skeleton equations}

\subsection{Existence and uniqueness of solutions to the skeleton equations}

Fix $h \in S_{M}$, and assume $h(t)=\sum_{k \geq 1} h^{k}(t) e_{k}$, where $\left\{e_{k}\right\}_{k \geq 1}$ is an orthonormal basis of $U$. Now, we introduce definitions of solution to the skeleton equation (3.18).

Definition 4.1. (Kinetic solution) Let $u_{0} \in L^{\infty}\left(\mathbb{T}^{N}\right)$. A measurable function $u_{h}: \mathbb{T}^{N} \times[0, T] \rightarrow \mathbb{R}$ is said to be a kinetic solution to (3.18), if for any $p \geq 1$, there exists $C_{p} \geq 0$ such that

$$
\text { ess } \sup _{t \in[0, T]}\left\|u_{h}(t)\right\|_{L^{p}\left(\mathbb{T}^{N}\right)}^{p} \leq C_{p}
$$

and if there exists a measure $m_{h} \in \mathcal{M}_{0}^{+}\left(\mathbb{T}^{N} \times[0, T] \times \mathbb{R}\right)$ such that $f_{h}:=I_{u_{h}>\xi}$ satisfies that for all $\varphi \in C_{c}^{1}\left(\mathbb{T}^{N} \times[0, T] \times \mathbb{R}\right)$,

$$
\begin{aligned}
& \int_{0}^{T}\left\langle f_{h}(t), \partial_{t} \varphi(t)\right\rangle d t+\left\langle f_{0}, \varphi(0)\right\rangle+\int_{0}^{T}\left\langle f_{h}(t), a(\xi) \cdot \nabla \varphi(t)\right\rangle d t \\
& \quad=-\sum_{k \geq 1} \int_{0}^{T} \int_{\mathbb{T}^{N}} g_{k}\left(x, u_{h}(x, t)\right) \varphi\left(x, t, u_{h}(x, t)\right) h^{k}(t) d x d t+m_{h}\left(\partial_{\xi} \varphi\right)
\end{aligned}
$$

where $f_{0}(x, \xi)=I_{u_{0}(x)>\xi}$. 
Definition 4.2. (Generalized kinetic solution) Let $f_{0}: \mathbb{T}^{N} \times \mathbb{R} \rightarrow[0,1]$ be a kinetic function. A measurable function $f_{h}: \mathbb{T}^{N} \times[0, T] \times \mathbb{R} \rightarrow[0,1]$ is said to be a generalized kinetic solution to (3.18) with the initial datum $f_{0}$, if $\left(f_{h}(t)\right)=\left(f_{h}(t, \cdot, \cdot)\right)$ is a kinetic function such that for all $p \geq 1, v^{h}:=-\partial_{\xi} f_{h}$ satisfies

$$
\text { ess } \sup _{t \in[0, T]} \int_{\mathbb{T}^{N}} \int_{\mathbb{R}}|\xi|^{p} d v_{x, t}^{h}(\xi) d x \leq C_{p},
$$

where $C_{p}$ is a positive constant and there exists a measure $m_{h} \in \mathcal{M}_{0}^{+}\left(\mathbb{T}^{N} \times[0, T] \times \mathbb{R}\right)$ such that for all $\varphi \in C_{c}^{1}\left(\mathbb{T}^{N} \times[0, T] \times \mathbb{R}\right)$,

$$
\begin{aligned}
& \int_{0}^{T}\left\langle f_{h}(t), \partial_{t} \varphi(t)\right\rangle d t+\left\langle f_{0}, \varphi(0)\right\rangle+\int_{0}^{T}\left\langle f_{h}(t), a(\xi) \cdot \nabla \varphi(t)\right\rangle d t \\
& \quad=-\sum_{k \geq 1} \int_{0}^{T} \int_{\mathbb{T}^{N}} \int_{\mathbb{R}} g_{k}(x, \xi) \varphi(x, t, \xi) h^{k}(t) d v_{x, t}^{h}(\xi) d x d t+m_{h}\left(\partial_{\xi} \varphi\right) .
\end{aligned}
$$

Theorem 4.1. (Existence) Let $u_{0} \in L^{\infty}\left(\mathbb{T}^{N}\right)$. Assume Hypothesis H holds, then for any $T>0$, (3.18) has a generalized kinetic solution $f_{h}$ with initial datum $f_{0}=I_{u_{0}>\xi}$.

The proof of Theorem 4.1 is similar to the proof of Theorem 2.5 which was done in [9], we therefore omit it here. Moreover, as stated in Proposition 2.1, for the generalized solution $f_{h}$, we have $f_{h}=f_{h}^{+}=f_{h}^{-}$ a.e. $t \in[0, T]$.

Taking a test function of the form $(x, s, \xi) \rightarrow \varphi(x, \xi) \alpha(s)$ in (4.21), where $\varphi \in C_{c}^{1}\left(\mathbb{T}^{N} \times \mathbb{R}\right)$ and $\alpha$ is the function

$$
\alpha(s)= \begin{cases}1, & s \leq t, \\ 1-\frac{s-t}{\varepsilon}, & t \leq s \leq t+\varepsilon, \\ 0, & t+\varepsilon \leq s,\end{cases}
$$

and letting $\varepsilon \rightarrow 0$, we obtain for all $t \in[0, T]$,

$$
\begin{aligned}
& -\left\langle f_{h}^{+}(t), \varphi\right\rangle+\left\langle f_{0}, \varphi\right\rangle+\int_{0}^{t}\left\langle f_{h}(s), a(\xi) \cdot \nabla \varphi\right\rangle d s \\
& =-\sum_{k \geq 1} \int_{0}^{t} \int_{\mathbb{T}^{N}} \int_{\mathbb{R}} g_{k}(x, \xi) \varphi(x, \xi) h^{k}(s) d v_{x, s}^{h}(\xi) d x d s+\left\langle m_{h}, \partial_{\xi} \varphi\right\rangle([0, t]), \text { a.s. }
\end{aligned}
$$

where $\left\langle m_{h}, \partial_{\xi} \varphi\right\rangle([0, t])=\int_{\mathbb{T}^{N} \times[0, t] \times \mathbb{R}} \partial_{\xi} \varphi(x, \xi) d m_{h}(x, s, \xi)$.

For the uniqueness, we firstly prove a comparison theorem for two generalized solutions $f_{i}, i=1,2$ of the following equations

$$
\left\{\begin{array}{l}
d u_{h}^{i}+\operatorname{div}\left(A\left(u_{h}^{i}\right)\right) d t=\Phi\left(u_{h}^{i}\right) h(t) d t \\
u_{h}^{i}(0)=u_{0}
\end{array}\right.
$$


Proposition 4.2. Under Hypothesis $H$, let $f_{i}, i=1,2$ be two generalized solutions to (4.24). Then, for $0 \leq t \leq T$, and nonnegative test functions $\rho \in C^{\infty}\left(\mathbb{T}^{N}\right), \psi \in C_{c}^{\infty}(\mathbb{R})$, we have

$$
\begin{aligned}
& \int_{\left(\mathbb{T}^{N}\right)^{2}} \int_{\mathbb{R}^{2}} \rho(x-y) \psi(\xi-\zeta)\left(f_{1}^{ \pm}(x, t, \xi) \bar{f}_{2}^{ \pm}(y, t, \zeta)+\bar{f}_{1}^{ \pm}(x, t, \xi) f_{2}^{ \pm}(y, t, \zeta)\right) d \xi d \zeta d x d y \\
\leq & \int_{\left(\mathbb{T}^{N}\right)^{2}} \int_{\mathbb{R}^{2}} \rho(x-y) \psi(\xi-\zeta)\left(f_{1,0}(x, \xi) \bar{f}_{2,0}(y, \zeta)+\bar{f}_{1,0}(x, \xi) f_{2,0}(y, \zeta)\right) d \xi d \zeta d x d y \\
& +K_{1}+\bar{K}_{1}+2 K_{2},
\end{aligned}
$$

where

$$
\begin{aligned}
& K_{1}=\int_{0}^{t} \int_{\left(\mathbb{T}^{N}\right)^{2}} \int_{\mathbb{R}^{2}} f_{1}(x, s, \xi) \bar{f}_{2}(y, s, \zeta)(a(\xi)-a(\zeta)) \nabla_{x} \alpha d \xi d \zeta d x d y d s \\
& \bar{K}_{1}=\int_{0}^{t} \int_{\left(\mathbb{T}^{N}\right)^{2}} \int_{\mathbb{R}^{2}} \bar{f}_{1}(x, s, \xi) f_{2}(y, s, \zeta)(a(\xi)-a(\zeta)) \nabla_{x} \alpha d \xi d \zeta d x d y d s
\end{aligned}
$$

and

$$
K_{2}=\sum_{k \geq 1} \int_{0}^{t} \int_{\left(\mathbb{T}^{N}\right)^{2}} \rho(x-y) \int_{\mathbb{R}^{2}} \gamma_{1}(\xi, \zeta)\left(g_{k}(x, \xi)-g_{k}(y, \zeta)\right) h^{k}(s) d v_{x, s}^{1} \otimes d v_{y, s}^{2}(\xi, \zeta) d x d y d s
$$

with $\gamma_{1}(\xi, \zeta)=\int_{-\infty}^{\xi} \psi\left(\xi^{\prime}-\zeta\right) d \xi^{\prime}=\int_{-\infty}^{\xi-\zeta} \psi(y) d y$.

Proof. Denote $f_{1}(x, t, \xi)=I_{u_{1}^{h}(x, t)>\xi}$ and $f_{2}(y, t, \zeta)=I_{u_{2}^{h}(y, t)>\zeta}$, respectively, with the corresponding kinetic measures $m_{1}$ and $m_{2}$. Let $\varphi_{1} \in C_{c}^{\infty}\left(\mathbb{T}_{x}^{N} \times \mathbb{R}_{\xi}\right)$ and $\varphi_{2} \in C_{c}^{\infty}\left(\mathbb{T}_{y}^{N} \times \mathbb{R}_{\zeta}\right)$. By (4.23), we have

$$
\begin{aligned}
\left\langle f_{1}^{+}(t), \varphi_{1}\right\rangle= & \left\langle f_{1,0}, \varphi_{1}\right\rangle+\int_{0}^{t}\left\langle f_{1}(s), a(\xi) \cdot \nabla_{x} \varphi_{1}(s)\right\rangle d s \\
& +\sum_{k \geq 1} \int_{0}^{t} \int_{\mathbb{T}^{N}} \int_{\mathbb{R}} g_{k}(x, \xi) \varphi_{1}(x, \xi) h^{k}(s) d v_{x, s}^{1}(\xi) d x d s-\left\langle m_{1}, \partial_{\xi} \varphi_{1}\right\rangle([0, t]),
\end{aligned}
$$

where $f_{1,0}=I_{u_{0}>\xi}$ and $v_{x, s}^{1}(\xi)=-\partial_{\xi} f_{1}^{+}(s, x, \xi)=\partial_{\xi} \bar{f}_{1}^{+}(s, x, \xi)$. Similarly,

$$
\begin{aligned}
\left\langle\bar{f}_{2}^{+}(t), \varphi_{2}\right\rangle= & \left\langle\bar{f}_{2,0}, \varphi_{2}\right\rangle+\int_{0}^{t}\left\langle\bar{f}_{2}(s), a(\zeta) \cdot \nabla_{y} \varphi_{2}(s)\right\rangle d s \\
& -\sum_{k \geq 1} \int_{0}^{t} \int_{\mathbb{T}^{N}} \int_{\mathbb{R}} g_{k}(y, \zeta) \varphi_{2}(y, \zeta) h^{k}(s) d v_{y, s}^{2}(\zeta) d y d s+\left\langle m_{2}, \partial_{\zeta} \varphi_{2}\right\rangle([0, t]) .
\end{aligned}
$$

where $f_{2,0}=I_{u_{0}>\zeta}$ and $v_{y, s}^{2}(\zeta)=\partial_{\zeta} \bar{f}_{2}^{+}(s, y, \zeta)=-\partial_{\zeta} f_{2}^{+}(s, y, \zeta)$.

Denote the duality distribution over $\mathbb{T}_{x}^{N} \times \mathbb{R}_{\xi} \times \mathbb{T}_{y}^{N} \times \mathbb{R}_{\zeta}$ by $\langle\langle\cdot, \cdot\rangle\rangle$. Setting $\alpha(x, \xi, y, \zeta)=\varphi_{1}(x, \xi) \varphi_{2}(y, \zeta)$ 
and using the integration by parts formula, we have

$$
\begin{aligned}
\left\langle\left\langle f_{1}^{+}(t) \bar{f}_{2}^{+}(t), \alpha\right\rangle\right\rangle= & \left\langle\left\langle f_{1,0} \bar{f}_{2,0}, \alpha\right\rangle\right\rangle+\int_{0}^{t} \int_{\left(\mathbb{T}^{N}\right)^{2}} \int_{\mathbb{R}^{2}} f_{1} \bar{f}_{2}\left(a(\xi) \cdot \nabla_{x}+a(\zeta) \cdot \nabla_{y}\right) \alpha d \xi d \zeta d x d y d s \\
& -\sum_{k \geq 1} \int_{0}^{t} \int_{\left(\mathbb{T}^{N}\right)^{2}} \int_{\mathbb{R}^{2}} f_{1}^{+}(s, x, \xi) \alpha g_{k}(y, \zeta) h^{k}(s) d \xi d v_{y, s}^{2}(\zeta) d x d y d s \\
& +\sum_{k \geq 1} \int_{0}^{t} \int_{\left(\mathbb{T}^{N}\right)^{2}} \int_{\mathbb{R}^{2}} \bar{f}_{2}^{+}(s, y, \zeta) \alpha g_{k}(x, \xi) h^{k}(s) d \zeta d v_{x, s}^{1}(\xi) d x d y d s \\
& +\int_{0}^{t} \int_{\left(\mathbb{T}^{N}\right)^{2}} \int_{\mathbb{R}^{2}} f_{1}^{+}(s, x, \xi) \partial_{\zeta} \alpha d m_{2}(y, \zeta, s) d \xi d x \\
& -\int_{0}^{t} \int_{\left.\mathbb{T}^{N}\right)^{2}} \int_{\mathbb{R}^{2}} \bar{f}_{2}^{+}(s, y, \zeta) \partial_{\xi} \alpha d m_{1}(x, \xi, s) d \zeta d y \\
:= & \left\langle\left\langle f_{1,0} \bar{f}_{2,0}, \alpha\right\rangle\right\rangle+I_{1}+I_{2}+I_{3}+I_{4}+I_{5} .
\end{aligned}
$$

Similarly, we have

$$
\begin{aligned}
\left\langle\left\langle\bar{f}_{1}^{+}(t) f_{2}^{+}(t), \alpha\right\rangle\right\rangle= & \left\langle\left\langle\bar{f}_{1,0} f_{2,0}, \alpha\right\rangle\right\rangle+\int_{0}^{t} \int_{\left(\mathbb{T}^{N}\right)^{2}} \int_{\mathbb{R}^{2}} \bar{f}_{1} f_{2}\left(a(\xi) \cdot \nabla_{x}+a(\zeta) \cdot \nabla_{y}\right) \alpha d \xi d \zeta d x d y d s \\
& +\sum_{k \geq 1} \int_{0}^{t} \int_{\left(\mathbb{T}^{N}\right)^{2}} \int_{\mathbb{R}^{2}} \bar{f}_{1}^{+}(s, x, \xi) \alpha g_{k}(y, \zeta) h^{k}(s) d \xi d v_{y, s}^{2}(\zeta) d x d y d s \\
& -\sum_{k \geq 1} \int_{0}^{t} \int_{\left(\mathbb{T}^{N}\right)^{2}} \int_{\mathbb{R}^{2}} f_{2}^{+}(s, y, \zeta) \alpha g_{k}(x, \xi) h^{k}(s) d v_{x, s}^{1}(\xi) d \zeta d x d y d s \\
& -\int_{0}^{t} \int_{\left(\mathbb{T}^{N}\right)^{2}} \int_{\mathbb{R}^{2}} \bar{f}_{1}^{+}(s, x, \xi) \partial_{\zeta} \alpha d m_{2}(y, \zeta, s) d \xi d x \\
& +\int_{0}^{t} \int_{\left(\mathbb{T}^{N}\right)^{2}} \int_{\mathbb{R}^{2}} f_{2}^{+}(s, y, \zeta) \partial_{\xi} \alpha d m_{1}(x, \xi, s) d \zeta d y \\
:= & \left\langle\left\langle\bar{f}_{1,0} f_{2,0}, \alpha\right\rangle\right\rangle+\bar{I}_{1}+\bar{I}_{2}+\bar{I}_{3}+\bar{I}_{4}+\bar{I}_{5} .
\end{aligned}
$$

By a density argument, (4.26) and (4.27) remain true for any test function $\alpha \in C_{c}^{\infty}\left(\mathbb{T}_{x}^{N} \times \mathbb{R}_{\xi} \times \mathbb{T}_{y}^{N} \times \mathbb{R}_{\zeta}\right)$. The assumption that $\alpha$ is compactly supported can be relaxed thanks to (2.2) on $m_{i}$ and (2.4) on $v_{i}$, $i=1,2$. Using a truncation argument of $\alpha$, it is easy to see that (4.26) and (4.27) remain true if $\alpha \in$ $C_{b}^{\infty}\left(\mathbb{T}_{x}^{N} \times \mathbb{R}_{\xi} \times \mathbb{T}_{y}^{N} \times \mathbb{R}_{\zeta}\right)$ is compactly supported in a neighbourhood of the diagonal

$$
\left\{(x, \xi, x, \xi) ; x \in \mathbb{T}^{N}, \xi \in \mathbb{R}\right\} .
$$

Taking $\alpha=\rho(x-y) \psi(\xi-\zeta)$, then we have the following remarkable identities

$$
\left(\nabla_{x}+\nabla_{y}\right) \alpha=0, \quad\left(\partial_{\xi}+\partial_{\zeta}\right) \alpha=0
$$

Referring to Proposition 13 in [9], we know that $I_{4}, I_{5}, \bar{I}_{4}, \bar{I}_{5}$ in (4.26) and (4.27) are all non-positive. As 
a result, we have

$$
\begin{aligned}
& \int_{\left(\mathbb{T}^{N}\right)^{2}} \int_{\mathbb{R}^{2}} \rho_{\gamma}(x-y) \psi_{\delta}(\xi-\zeta)\left(f_{1}^{+}(x, t, \xi) \bar{f}_{2}^{+}(y, t, \zeta)+\bar{f}_{1}^{+}(x, t, \xi) f_{2}^{+}(y, t, \zeta)\right) d \xi d \zeta d x d y \\
\leq & \int_{\left(\mathbb{T}^{N}\right)^{2}} \int_{\mathbb{R}^{2}} \rho_{\gamma}(x-y) \psi_{\delta}(\xi-\zeta)\left(f_{1,0}(x, \xi) \bar{f}_{2,0}(y, \zeta)+\bar{f}_{1,0}(x, \xi) f_{2,0}(y, \zeta)\right) d \xi d \zeta d x d y \\
& +\sum_{i=1}^{3}\left(I_{i}+\bar{I}_{i}\right) .
\end{aligned}
$$

With the aid of (4.28), we deduce that

$$
\begin{aligned}
& I_{1}=\int_{0}^{t} \int_{\left(\mathbb{T}^{N}\right)^{2}} \int_{\mathbb{R}^{2}} f_{1} \bar{f}_{2}(a(\xi)-a(\zeta)) \nabla_{x} \alpha d \xi d \zeta d x d y d s, \\
& \bar{I}_{1}=\int_{0}^{t} \int_{\left(\mathbb{T}^{N}\right)^{2}} \int_{\mathbb{R}^{2}} \bar{f}_{1} f_{2}(a(\xi)-a(\zeta)) \nabla_{x} \alpha d \xi d \zeta d x d y d s .
\end{aligned}
$$

Let

$$
\gamma_{1}(\xi, \zeta)=\int_{-\infty}^{\xi} \psi\left(\xi^{\prime}-\zeta\right) d \xi^{\prime}
$$

for some $\xi, \zeta \in \mathbb{R}$. Then

$$
\begin{aligned}
I_{2} & =-\sum_{k \geq 1} \int_{0}^{t} \int_{\left(\mathbb{T}^{N}\right)^{2}} \int_{\mathbb{R}^{2}} f_{1}^{+}(s, x, \xi) \rho(x-y) \partial_{\xi} \gamma_{1}(\xi, \zeta) g_{k}(y, \zeta) h^{k}(s) d \xi d v_{y, s}^{2}(\zeta) d x d y d s \\
& =-\sum_{k \geq 1} \int_{0}^{t} \int_{\left(\mathbb{T}^{N}\right)^{2}} \int_{\mathbb{R}} \rho(x-y) g_{k}(y, \zeta) h^{k}(s)\left(\int_{\mathbb{R}} f_{1}^{+}(s, x, \xi) \partial_{\xi} \gamma_{1}(\xi, \zeta) d \xi\right) d v_{y, s}^{2}(\zeta) d x d y d s \\
& =-\sum_{k \geq 1} \int_{0}^{t} \int_{\left(\mathbb{T}^{N}\right)^{2}} \int_{\mathbb{R}^{2}} \rho(x-y) \gamma_{1}(\xi, \zeta) g_{k}(y, \zeta) h^{k}(s) d v_{x, s}^{1} \otimes d v_{y, s}^{2}(\xi, \zeta) d x d y d s .
\end{aligned}
$$

The third equality is obtained by

$$
\begin{aligned}
\int_{\mathbb{R}} f_{1}^{+}(s, x, \xi) \partial_{\xi} \gamma_{1}(\xi, \zeta) d \xi & =-\int_{\mathbb{R}} \partial_{\xi} f_{1}^{+}(s, x, \xi) \gamma_{1}(\xi, \zeta) d \xi \\
& =\int_{\mathbb{R}} \gamma_{1}(\xi, \zeta) d v_{x, s}^{1}(\xi) .
\end{aligned}
$$

Similarly, for $\xi, \zeta \in \mathbb{R}$, let

$$
\gamma_{2}(\zeta, \xi)=\int_{\zeta}^{\infty} \psi\left(\xi-\zeta^{\prime}\right) d \zeta^{\prime}
$$

then

$$
\begin{aligned}
I_{3} & =-\sum_{k \geq 1} \int_{0}^{t} \int_{\left(\mathbb{T}^{N}\right)^{2}} \int_{\mathbb{R}^{2}} \bar{f}_{2}^{+}(s, y, \zeta) \rho(x-y) \partial_{\zeta} \gamma_{2}(\zeta, \xi) g_{k}(x, \xi) h^{k}(s) d v_{x, s}^{1}(\xi) d \zeta d x d y d s \\
& =-\sum_{k \geq 1} \int_{0}^{t} \int_{\left(\mathbb{T}^{N}\right)^{2}} \int_{\mathbb{R}} \rho(x-y) g_{k}(x, \xi) h^{k}(s)\left(\int_{\mathbb{R}} \bar{f}_{2}^{+}(s, y, \zeta) \partial_{\zeta} \gamma_{2}(\zeta, \xi) d \zeta\right) d v_{x, s}^{1}(\xi) d x d y d s \\
& =\sum_{k \geq 1} \int_{0}^{t} \int_{\left(\mathbb{T}^{N}\right)^{2}} \int_{\mathbb{R}^{2}} \gamma_{2}(\zeta, \xi) \rho(x-y) g_{k}(x, \xi) h^{k}(s) d v_{x, s}^{1} \otimes d v_{y, s}^{2}(\xi, \zeta) d x d y d s
\end{aligned}
$$


Note that $\gamma_{1}(\xi, \zeta)=\gamma_{2}(\zeta, \xi)=\int_{-\infty}^{\xi-\zeta} \psi(y) d y$. We deduce from (4.29) and (4.30) that

$$
I_{2}+I_{3}=\sum_{k \geq 1} \int_{0}^{t} \int_{\left(\mathbb{T}^{N}\right)^{2}} \rho(x-y) \int_{\mathbb{R}^{2}} \gamma_{1}(\xi, \zeta)\left(g_{k}(x, \xi)-g_{k}(y, \zeta)\right) h^{k}(s) d v_{x, s}^{1} \otimes d v_{y, s}^{2}(\xi, \zeta) d x d y d s
$$

Similarly, we have

$$
\bar{I}_{2}+\bar{I}_{3}=\sum_{k \geq 1} \int_{0}^{t} \int_{\left(\mathbb{T}^{N}\right)^{2}} \rho(x-y) \int_{\mathbb{R}^{2}} \gamma_{1}(\xi, \zeta)\left(g_{k}(x, \xi)-g_{k}(y, \zeta)\right) h^{k}(s) d v_{x, s}^{1} \otimes d v_{y, s}^{2}(\xi, \zeta) d x d y d s
$$

Taking $K_{1}=I_{1}, \bar{K}_{1}=\bar{I}_{1}$ and $K_{2}=I_{2}+I_{3}=\bar{I}_{2}+\bar{I}_{3}$, the equation (4.25) is established for $f_{i}^{+}$. To obtain the result for $f_{i}^{-}$, we take $t_{n} \uparrow t$, write (4.25) for $f_{i}^{+}\left(t_{n}\right)$ and let $n \rightarrow \infty$.

Theorem 4.3. Let $u_{0} \in L^{\infty}\left(\mathbb{T}^{N}\right)$ and assume Hypothesis $H$ holds. Then there exists at most one kinetic solution to (3.18) with the initial datum $u_{0}$. Besides, any generalized solution $f_{h}$ is actually a kinetic solution, i.e. if $f_{h}$ is a generalized solution to (3.18) with initial datum $I_{u_{0}>\xi}$, then there exists a kinetic solution $u_{h}$ to (3.18) with initial datum $u_{0}$ such that $f_{h}(x, t, \xi)=I_{u_{h}(x, t)>\xi}$, for a.e. $(x, t, \xi)$.

Proof. Let $\rho_{\gamma}, \psi_{\delta}$ be approximations to the identity on $\mathbb{T}^{N}$ and $\mathbb{R}$, respectively. That is, let $\rho \in C^{\infty}\left(\mathbb{T}^{N}\right)$, $\psi \in C_{c}^{\infty}(\mathbb{R})$ be symmetric nonnegative functions such as $\int_{\mathbb{T}^{N}} \rho=1, \int_{\mathbb{R}} \psi=1$ and $\operatorname{supp} \psi \subset(-1,1)$. We define

$$
\rho_{\gamma}(x)=\frac{1}{\gamma^{N}} \rho\left(\frac{x}{\gamma}\right), \quad \psi_{\delta}(\xi)=\frac{1}{\delta} \psi\left(\frac{\xi}{\delta}\right) .
$$

Letting $\rho:=\rho_{\gamma}(x-y)$ and $\psi:=\psi_{\delta}(\xi-\zeta)$ in Proposition 4.2, we get from (4.25) that

$$
\begin{aligned}
& \int_{\left(\mathbb{T}^{N}\right)^{2}} \int_{\mathbb{R}^{2}} \rho_{\gamma}(x-y) \psi_{\delta}(\xi-\zeta)\left(f_{1}^{ \pm}(x, t, \xi) \bar{f}_{2}^{ \pm}(y, t, \zeta)+\bar{f}_{1}^{ \pm}(x, t, \xi) f_{2}^{ \pm}(y, t, \zeta)\right) d \xi d \zeta d x d y \\
\leq & \int_{\left(\mathbb{T}^{N}\right)^{2}} \int_{\mathbb{R}^{2}} \rho_{\gamma}(x-y) \psi_{\delta}(\xi-\zeta)\left(f_{1,0}(x, \xi) \bar{f}_{2,0}(y, \zeta)+\bar{f}_{1,0}(x, \xi) f_{2,0}(y, \zeta)\right) d \xi d \zeta d x d y \\
& +\tilde{K}_{1}+\tilde{K}_{1}+2 \tilde{K}_{2},
\end{aligned}
$$

where $\tilde{K}_{1}, \tilde{K}_{1}, \tilde{K}_{2}$ in (4.31) are the corresponding $K_{1}, \bar{K}_{1}, K_{2}$ in the statement of Proposition 4.2 with $\rho$, $\psi$ replaced by $\rho_{\gamma}, \psi_{\delta}$, respectively. Let $\tilde{\gamma}_{1}(\xi, \zeta)=\int_{-\infty}^{\xi} \psi_{\delta}\left(\xi^{\prime}-\zeta\right) d \xi^{\prime}$, for simplicity, we denote $\tilde{\gamma}_{1}(\xi, \zeta)=$ $\gamma_{1}(\xi, \zeta)$. In the following, we devote to making estimates of $\tilde{K}_{1}, \tilde{K}_{1}$ and $\tilde{K}_{2}$.

For $\tilde{K}_{1}$ and $\tilde{\bar{K}}_{1}$, by (2.11) and using the same method as the proof of Theorem 15 in [9], we have

$$
\left|\tilde{K}_{1}\right|+\left|\tilde{\bar{K}}_{1}\right| \leq 2 T C_{p} \delta \gamma^{-1}
$$


For $\tilde{K}_{2}$, by utilizing (2.13), we deduce that

$$
\begin{aligned}
\tilde{K}_{2} \leq & \int_{0}^{t} \int_{\left(\mathbb{T}^{N}\right)^{2}} \rho_{\gamma}(x-y) \int_{\mathbb{R}^{2}} \gamma_{1}(\xi, \zeta) \sum_{k \geq 1}\left|g_{k}(x, \xi)-g_{k}(y, \zeta) \| h^{k}(s)\right| d v_{x, s}^{1} \otimes d v_{y, s}^{2}(\xi, \zeta) d x d y d s \\
\leq & \int_{0}^{t} \int_{\left(\mathbb{T}^{N}\right)^{2}} \rho_{\gamma}(x-y) \int_{\mathbb{R}^{2}} \gamma_{1}(\xi, \zeta)\left(\sum_{k \geq 1} \mid g_{k}(x, \xi)-g_{k}(y, \zeta)^{2}\right)^{\frac{1}{2}}\left(\sum_{k \geq 1}\left|h^{k}(s)\right|^{2}\right)^{\frac{1}{2}} d v_{x, s}^{1} \otimes d v_{y, s}^{2}(\xi, \zeta) d x d y d s \\
\leq & \sqrt{D_{1}} \int_{0}^{t}|h(s)|_{U} \int_{\left(\mathbb{T}^{N}\right)^{2}} \rho_{\gamma}(x-y)|x-y| \int_{\mathbb{R}^{2}} \gamma_{1}(\xi, \zeta) d v_{x, s}^{1} \otimes d v_{y, s}^{2}(\xi, \zeta) d x d y d s \\
& +\sqrt{D_{1}} \int_{0}^{t}|h(s)|_{U} \int_{\left(\mathbb{T}^{N}\right)^{2}} \rho_{\gamma}(x-y) \int_{\mathbb{R}^{2}} \gamma_{1}(\xi, \zeta)|\xi-\zeta| d v_{x, s}^{1} \otimes d v_{y, s}^{2}(\xi, \zeta) d x d y d s \\
:= & \tilde{K}_{2,1}+\tilde{K}_{2,2},
\end{aligned}
$$

Note that

$$
\begin{aligned}
\int_{\mathbb{R}^{2}} \gamma_{1}(\xi, \zeta) d v_{x, s}^{1} \otimes d v_{y, s}^{2}(\xi, \zeta) & \leq 1 \\
\int_{\left(\mathbb{T}^{N}\right)^{2}} \rho_{\gamma}(x-y)|x-y| d x d y & \leq \gamma,
\end{aligned}
$$

it implies that

$$
\tilde{K}_{2,1} \leq \sqrt{D_{1}} \gamma \int_{0}^{t}|h(s)|_{U} d s \leq \sqrt{D_{1}} \gamma(T+M) .
$$

Moreover, by $v_{x, s}^{1}(\xi)=-\partial_{\xi} f_{1}^{ \pm}(s, x, \xi)=\partial_{\xi} \bar{f}_{1}^{ \pm}(s, x, \xi)=\delta_{u_{h}^{1, \pm}(x, s)=\xi}$ and $v_{y, s}^{2}(\zeta)=\partial_{\zeta} \bar{f}_{2}^{ \pm}(s, y, \zeta)=$ $-\partial_{\zeta} f_{2}^{ \pm}(s, y, \zeta)=\delta_{u_{h}^{2, \pm}(y, s)=\zeta}$, it follows that

$$
\begin{aligned}
\tilde{K}_{2,2} \leq & \sqrt{D_{1}} \int_{0}^{t}|h(s)|_{U} \int_{\left(\mathbb{T}^{N}\right)^{2}} \int_{\mathbb{R}^{2}} \rho_{\gamma}(x-y)|\xi-\zeta| d v_{x, s}^{1} \otimes d v_{y, s}^{2}(\xi, \zeta) d x d y d s \\
= & \sqrt{D_{1}} \int_{0}^{t}|h(s)|_{U} \int_{\left.\mathbb{T}^{N}\right)^{2}} \rho_{\gamma}(x-y)\left|u_{h}^{1, \pm}(x, s)-u_{h}^{2, \pm}(y, s)\right| d x d y d s \\
= & \sqrt{D_{1}} \int_{0}^{t}|h(s)|_{U} \int_{\left(\mathbb{T}^{N}\right)^{2}} \rho_{\gamma}(x-y)\left(u_{h}^{1, \pm}(x, s)-u_{h}^{2, \pm}(y, s)\right)^{+} d x d y d s \\
& +\sqrt{D_{1}} \int_{0}^{t}|h(s)|_{U} \int_{\left(\mathbb{T}^{N}\right)^{2}} \rho_{\gamma}(x-y)\left(u_{h}^{1, \pm}(x, s)-u_{h}^{2, \pm}(y, s)\right)^{-} d x d y d s \\
= & \sqrt{D_{1}} \int_{0}^{t}|h(s)|_{U} \int_{\left(\mathbb{T}^{N}\right)^{2}} \int_{\mathbb{R}} \rho_{\gamma}(x-y)\left(f_{1}^{ \pm}(x, s, \xi) \bar{f}_{2}^{ \pm}(y, s, \xi)+\bar{f}_{1}^{ \pm}(x, s, \xi) f_{2}^{ \pm}(y, s, \xi)\right) d \xi d x d y d s .
\end{aligned}
$$


By utilizing $\int_{\mathbb{R}} \psi_{\delta}(\xi-\zeta) d \zeta=1$ and $\int_{\xi-\delta}^{\xi} \psi_{\delta}(\xi-\zeta) d \zeta=\frac{1}{2}$, we get

$$
\begin{aligned}
& \mid \int_{\left(\mathbb{T}^{N}\right)^{2}} \int_{\mathbb{R}} \rho_{\gamma}(x-y) f_{1}^{ \pm}(x, s, \xi) \bar{f}_{2}^{ \pm}(y, s, \xi) d \xi d x d y \\
& -\int_{\left(\mathbb{T}^{N}\right)^{2}} \int_{\mathbb{R}^{2}} f_{1}^{ \pm}(x, s, \xi) \bar{f}_{2}^{ \pm}(y, s, \zeta) \rho_{\gamma}(x-y) \psi_{\delta}(\xi-\zeta) d x d y d \xi d \zeta \mid \\
= & \left|\int_{\left(\mathbb{T}^{N}\right)^{2}} \rho_{\gamma}(x-y) \int_{\mathbb{R}} I_{u_{h}^{1, \pm}(x, s)>\xi} \int_{\mathbb{R}} \psi_{\delta}(\xi-\zeta)\left(I_{u_{h}^{2, \pm}(y, s) \leq \xi}-I_{u_{h}^{2, \pm}(y, s) \leq \zeta}\right) d \zeta d \xi d x d y\right| \\
\leq & \int_{\left(\mathbb{T}^{N}\right)^{2}} \int_{\mathbb{R}} \rho_{\gamma}(x-y) I_{u_{h}^{1, \pm}(x, s)>\xi} \int_{\xi-\delta}^{\xi} \psi_{\delta}(\xi-\zeta) I_{\zeta<u_{h}^{2, \pm}(y, s) \leq \xi} d \zeta d \xi d x d y \\
& +\int_{\left(\mathbb{T}^{N}\right)^{2}} \int_{\mathbb{R}} \rho_{\gamma}(x-y) I_{u_{h}^{1, \pm}(x, s)>\xi} \int_{\xi}^{\xi+\delta} \psi_{\delta}(\xi-\zeta) I_{\xi<u_{h}^{2, \pm}(y, s) \leq \zeta} d \zeta d \xi d x d y \mid \\
\leq & \frac{1}{2} \int_{\left(\mathbb{T}^{N}\right)^{2}} \rho_{\gamma}(x-y) \int_{u_{h}^{2, \pm}(y, s)}^{\min \left\{u_{h}^{1, \pm}(x, s), u_{h}^{2, \pm}(y, s)+\delta\right\}} d \xi d x d y \\
& +\frac{1}{2} \int_{\left(\mathbb{T}^{N}\right)^{2}} \rho_{\gamma}(x-y) \int_{u_{h}^{2, \pm}(y, s)-\delta}^{\min \left\{u_{h}^{1, \pm}(x, s), u_{h}^{2, \pm}(y, s)\right\}} d \xi d x d y \\
\leq & \frac{1}{2} \delta+\frac{1}{2} \delta=\delta .
\end{aligned}
$$

Similarly, we deduce that

$$
\begin{aligned}
& \mid \int_{\left(\mathbb{T}^{N}\right)^{2}} \int_{\mathbb{R}} \rho_{\gamma}(x-y) \bar{f}_{1}^{ \pm}(x, s, \xi) f_{2}^{ \pm}(y, s, \xi) d \xi d x d y \\
& -\int_{\left(\mathbb{T}^{N}\right)^{2}} \int_{\mathbb{R}^{2}} \bar{f}_{1}^{ \pm}(x, s, \xi) f_{2}^{ \pm}(y, s, \zeta) \rho_{\gamma}(x-y) \psi_{\delta}(\xi-\zeta) d x d y d \xi d \zeta \mid \\
= & \left|\int_{\left(\mathbb{T}^{N}\right)^{2}} \rho_{\gamma}(x-y) \int_{\mathbb{R}} I_{u_{h}^{1, \pm}(x, s) \leq \xi} \int_{\mathbb{R}} \psi_{\delta}(\xi-\zeta)\left(I_{u_{h}^{2, \pm}(y, s)>\xi}-I_{u_{h}^{2, \pm}(y, s)>\zeta}\right) d \zeta d \xi d x d y\right| \\
\leq & \int_{\left(\mathbb{T}^{N}\right)^{2}} \int_{\mathbb{R}} \rho_{\gamma}(x-y) I_{u_{h}^{1, \pm}(x, s) \leq \xi} \int_{\xi-\delta}^{\xi} \psi_{\delta}(\xi-\zeta) I_{\zeta<u_{h}^{2, \pm}(y, s) \leq \xi} d \zeta d \xi d x d y \\
& +\int_{\left(\mathbb{T}^{N}\right)^{2}} \int_{\mathbb{R}} \rho_{\gamma}(x-y) I_{u_{h}^{1, \pm}(x, s) \leq \xi} \int_{\xi}^{\xi+\delta} \psi_{\delta}(\xi-\zeta) I_{\xi<u_{h}^{2, \pm}(y, s) \leq \zeta} d \zeta d \xi d x d y \mid \\
\leq & \frac{1}{2} \int_{\left(\mathbb{T}^{N}\right)^{2}} \rho_{\gamma}(x-y) \int_{\max \left\{u_{h}^{1, \pm}(x, s), u_{h}^{2, \pm}(y, s)\right\}}^{u_{h}^{2, \pm}(y, s)+\delta} d \xi d x d y \\
& +\frac{1}{2} \int_{\left.\mathbb{T}^{N}\right)^{2}} \rho_{\gamma}(x-y) \int_{\max \left\{u_{h}^{1, \pm}(x, s), u_{h}^{2, \pm}(y, s)-\delta\right\}}^{u_{h}^{, \pm \pm}(y, s)} d \xi d x d y \\
\leq & \frac{1}{2} \delta+\frac{1}{2} \delta=\delta .
\end{aligned}
$$


Then, we deduce from (4.34) and (4.35) that

$$
\begin{aligned}
\tilde{K}_{2,2} \leq & 2 \delta \sqrt{D_{1}} \int_{0}^{t}|h(s)|_{U} d s \\
& +\sqrt{D_{1}} \int_{0}^{t}|h(s)|_{U} \int_{\left(\mathbb{T}^{N}\right)^{2}} \int_{\mathbb{R}^{2}}\left(f_{1}^{ \pm} \bar{f}_{2}^{ \pm}+\bar{f}_{1}^{ \pm} f_{2}^{ \pm}\right) \rho_{\gamma}(x-y) \psi_{\delta}(\xi-\zeta) d x d y d \xi d \zeta d s \\
\leq & 2 \delta \sqrt{D_{1}}(T+M) \\
& +\sqrt{D_{1}} \int_{0}^{t}|h(s)|_{U} \int_{\left(\mathbb{T}^{N}\right)^{2}} \int_{\mathbb{R}^{2}}\left(f_{1}^{ \pm} \bar{f}^{ \pm}+\bar{f}_{1}^{ \pm} f_{2}^{ \pm}\right) \rho_{\gamma}(x-y) \psi_{\delta}(\xi-\zeta) d x d y d \xi d \zeta d s .
\end{aligned}
$$

Hence, Combing (4.33) and (4.36), we get

$$
\begin{aligned}
\tilde{K}_{2} \leq & \sqrt{D_{1}}(\gamma+2 \delta)(T+M) \\
& +\sqrt{D_{1}} \int_{0}^{t}|h(s)|_{U} \int_{\left(\mathbb{T}^{N}\right)^{2}} \int_{\mathbb{R}^{2}}\left(f_{1}^{ \pm} \bar{f}_{2}^{ \pm}+\bar{f}_{1}^{ \pm} f_{2}^{ \pm}\right) \rho_{\gamma}(x-y) \psi_{\delta}(\xi-\zeta) d x d y d \xi d \zeta d s .
\end{aligned}
$$

Taking into account (4.31), we deduce that

$$
\begin{aligned}
& \int_{\left(\mathbb{T}^{N}\right)^{2}} \int_{\mathbb{R}^{2}} \rho_{\gamma}(x-y) \psi_{\delta}(\xi-\zeta)\left(f_{1}^{ \pm}(x, t, \xi) \bar{f}_{2}^{ \pm}(y, t, \zeta)+\bar{f}_{1}^{ \pm}(x, t, \xi) f_{2}^{ \pm}\right)(y, t, \zeta) d \xi d \zeta d x d y \\
\leq & \int_{\left(\mathbb{T}^{N}\right)^{2}} \int_{\mathbb{R}^{2}} \rho_{\gamma}(x-y) \psi_{\delta}(\xi-\zeta)\left(f_{1,0}(x, \xi) \bar{f}_{2,0}(y, \zeta)+\bar{f}_{1,0}(x, \xi) f_{2,0}(y, \zeta)\right) d \xi d \zeta d x d y \\
& +2 T C_{p} \delta \gamma^{-1}+2 \sqrt{D_{1}}(\gamma+2 \delta)(T+M) \\
& +2 \sqrt{D_{1}} \int_{0}^{t}|h(s)|_{U} \int_{\left(\mathbb{T}^{N}\right)^{2}} \int_{\mathbb{R}^{2}}\left(f_{1}^{ \pm} \bar{f}_{2}^{ \pm}+\bar{f}_{1}^{ \pm} f_{2}^{ \pm}\right) \rho_{\gamma}(x-y) \psi_{\delta}(\xi-\zeta) d x d y d \xi d \zeta d s \\
\leq & \int_{\mathbb{T}^{N}} \int_{\mathbb{R}}\left(f_{1,0} \bar{f}_{2,0}+\bar{f}_{1,0} f_{2,0}\right) d \xi d x+\mathcal{E}_{0}(\gamma, \delta)+2 T C_{p} \delta \gamma^{-1}+2 \sqrt{D_{1}}(\gamma+\delta)(T+M) \\
& +2 \sqrt{D_{1}} \int_{0}^{t}|h(s)|_{U} \int_{\left(\mathbb{T}^{N}\right)^{2}} \int_{\mathbb{R}^{2}}\left(f_{1}^{ \pm} \bar{f}_{2}^{ \pm}+\bar{f}_{1}^{ \pm} f_{2}^{ \pm}\right) \rho_{\gamma}(x-y) \psi_{\delta}(\xi-\zeta) d x d y d \xi d \zeta d s,
\end{aligned}
$$

where $\mathcal{E}_{0}(\gamma, \delta) \rightarrow 0$, as $\gamma, \delta \rightarrow 0$.

Utilizing Gronwall inequality, we obtain

$$
\begin{aligned}
& \int_{\left(\mathbb{T}^{N}\right)^{2}} \int_{\mathbb{R}^{2}} \rho_{\gamma}(x-y) \psi_{\delta}(\xi-\zeta)\left(f_{1}^{ \pm}(x, t, \xi) \bar{f}_{2}^{ \pm}(y, t, \zeta)+\bar{f}_{1}^{ \pm}(x, t, \xi) f_{2}^{ \pm}(y, t, \zeta)\right) d \xi d \zeta d x d y \\
\leq & {\left[\int_{\mathbb{T}^{N}} \int_{\mathbb{R}}\left(f_{1,0} \bar{f}_{2,0}+\bar{f}_{1,0} f_{2,0}\right) d \xi d x+\mathcal{E}_{0}(\gamma, \delta)+2 T C_{p} \delta \gamma^{-1}+2 \sqrt{D_{1}}(\gamma+2 \delta)(T+M)\right] } \\
& \times \exp \left\{2 \sqrt{D_{1}} \int_{0}^{t}|h(s)|_{U} d s\right\} \\
\leq & e^{2 \sqrt{D_{1}}(T+M)}\left[\int_{\mathbb{T}^{N}} \int_{\mathbb{R}}\left(f_{1,0} \bar{f}_{2,0}+\bar{f}_{1,0} f_{2,0}\right) d \xi d x+\mathcal{E}_{0}(\gamma, \delta)\right] \\
& +2 e^{2 \sqrt{D_{1}}(T+M)}\left[T C_{p} \delta \gamma^{-1}+\sqrt{D_{1}}(\gamma+2 \delta)(T+M)\right] .
\end{aligned}
$$


Combing all the above estimates, it follows that

$$
\begin{aligned}
& \int_{\mathbb{T}^{N}} \int_{\mathbb{R}}\left(f_{1}^{ \pm}(x, t, \xi) \bar{f}_{2}^{ \pm}(x, t, \xi)+\bar{f}_{1}^{ \pm}(x, t, \xi) f_{2}^{ \pm}(x, t, \xi)\right) d x d \xi \\
= & \int_{\left(\mathbb{T}^{N}\right)^{2}} \int_{\mathbb{R}^{2}} \rho_{\gamma}(x-y) \psi_{\delta}(\xi-\zeta)\left(f_{1}^{ \pm}(x, t, \xi) \bar{f}_{2}^{ \pm}(y, t, \zeta)+\bar{f}_{1}^{ \pm}(x, t, \xi) f_{2}^{ \pm}(y, t, \zeta)\right) d \xi d \zeta d x d y+\mathcal{E}_{t}(\gamma, \delta) \\
\leq & e^{2 \sqrt{D_{1}}(T+M)}\left[\int_{\mathbb{T}^{N}} \int_{\mathbb{R}}\left(f_{1,0} \bar{f}_{2,0}+\bar{f}_{1,0} f_{2,0}\right) d \xi d x+2 \mathcal{E}_{0}(\gamma, \delta)\right] \\
& +2 e^{2 \sqrt{D_{1}}(T+M)}\left[T C_{p} \delta \gamma^{-1}+\sqrt{D_{1}}(\gamma+2 \delta)(T+M)\right]+\mathcal{E}_{t}(\gamma, \delta),
\end{aligned}
$$

where $\mathcal{E}_{t}(\gamma, \delta) \rightarrow 0$, as $\gamma, \delta \rightarrow 0$.

Taking $\delta=\gamma^{\frac{4}{3}}$ and letting $\gamma \rightarrow 0$ gives

$$
\begin{aligned}
& \int_{\mathbb{T}^{N}} \int_{\mathbb{R}}\left(f_{1}^{ \pm}(x, t, \xi) \bar{f}_{2}^{ \pm}(x, t, \xi)+\bar{f}_{1}^{ \pm}(x, t, \xi) f_{2}^{ \pm}(x, t, \xi)\right) d x d \xi \\
\leq & e^{2 \sqrt{D_{1}}(T+M)} \int_{\mathbb{T}^{N}} \int_{\mathbb{R}}\left(f_{1,0} \bar{f}_{2,0}+\bar{f}_{1,0} f_{2,0}\right) d x d \xi .
\end{aligned}
$$

The reduction of generalized solutions to kinetic solutions is very similar to the proof of Theorem 15 in [9], we therefore omit it here. Suppose that $u_{h}^{1}$ and $u_{h}^{2}$ are two kinetic solutions to (4.24), using the following identities

$$
\int_{\mathbb{R}} I_{u_{h}^{1}>\xi} \overline{I_{u_{h}^{2}>\xi}} d \xi=\left(u_{h}^{1}-u_{h}^{2}\right)^{+}, \quad \int_{\mathbb{R}} \overline{I_{u_{h}^{1}>\xi}} I_{u_{h}^{2}>\xi} d \xi=\left(u_{h}^{1}-u_{h}^{2}\right)^{-},
$$

we deduce from (4.40) with $f_{i}=I_{u_{h}^{i}>\xi}, f_{i, 0}=I_{u_{0}>\xi}$ that

$$
\left\|u_{h}^{1}(t)-u_{h}^{2}(t)\right\|_{L^{1}\left(\mathbb{T}^{N}\right)} \leq e^{\sqrt{D_{1}}(T+M)}\left\|u_{0}-u_{0}\right\|_{L^{1}\left(\mathbb{T}^{N}\right)}=0 .
$$

This gives the uniqueness.

In view of Theorem 4.1 and Theorem 4.3, we can define $\mathcal{G}^{0}: C([0, T] ; \mathcal{U}) \rightarrow L^{1}\left([0, T] ; L^{1}\left(\mathbb{T}^{N}\right)\right)$ by

$$
\mathcal{G}^{0}(\breve{h}):= \begin{cases}u_{h}, & \text { if } \breve{h}=\int_{0} h(s) d s, \text { for some } h \in L^{2}([0, T] ; U), \\ 0, & \text { otherwise },\end{cases}
$$

where $u_{h}$ is the solution of equation (3.18).

\subsection{The continuity of the skeleton equations}

In this part, we aim to prove the continuity of $G^{0}$, i.e., the kinetic solution $u_{h^{\varepsilon}}$ of (4.24) converges to the kinetic solution $u_{h}$ of the skeleton equation (3.18) in $L^{1}\left([0, T] ; L^{1}\left(\mathbb{T}^{N}\right)\right)$, if $h^{\varepsilon} \rightarrow h$ weakly in $L^{2}([0, T] ; U)$. For technical needs, we will introduce two auxiliary approximation processes.

Firstly, for any family $\left\{h^{\varepsilon}, \varepsilon>0\right\} \subset S_{M}$ and $\eta>0$, we consider the following parabolic approximation

$$
\left\{\begin{array}{l}
d u_{h^{\varepsilon}}^{\eta}-\eta \Delta u_{h^{\varepsilon}}^{\eta} d t+\operatorname{div}\left(A\left(u_{h^{\varepsilon}}^{\eta}\right)\right) d t=\Phi\left(u_{h^{\varepsilon}}^{\eta}\right) h^{\varepsilon}(t) d t \\
u_{h^{\varepsilon}}^{\eta}(0)=u_{0} .
\end{array}\right.
$$


It is shown by Theorem 2.1 in [15] that equation (4.43) has a unique $L^{\varrho}\left(\mathbb{T}^{N}\right)$-valued solution provided $\varrho$ is large enough and $u_{0} \in L^{\varrho}\left(\mathbb{T}^{N}\right)$, hence in particular for $u_{0} \in L^{\infty}\left(\mathbb{T}^{N}\right)$. We denote by $u_{h^{\varepsilon}}^{\eta}$ the solution of (4.43).

Furthermore, for any $R \in \mathbb{N}$, we approximate operator $A$ in (4.43) by Lipschitz continuous operator $A^{R}$ using the method of truncation. Consider the following approximation

$$
\left\{\begin{array}{l}
d u_{h^{\varepsilon}}^{\eta, R}-\eta \Delta u_{h^{\varepsilon}}^{\eta, R} d t+\operatorname{div}\left(A^{R}\left(u_{h^{\varepsilon}}^{\eta, R}\right)\right) d t=\Phi\left(u_{h^{\varepsilon}}^{\eta, R}\right) h^{\varepsilon}(t) d t, \\
u_{h^{\varepsilon}}^{\eta, R}(0)=u_{0},
\end{array}\right.
$$

where $A^{R}$ is Lipschitz continuous hence it has linear growth $\left|A^{R}(\xi)\right| \leq C(R)(1+|\xi|)$.

Referring to Proposition 5.1 in [8], we have

$$
\sup _{\varepsilon}\left\{\sup _{t \in[0, T]}\left\|u_{h^{\varepsilon}}^{\eta, R}\right\|_{H}^{2}+\int_{0}^{T}\left\|\nabla u_{h^{\varepsilon}}^{\eta, R}(s)\right\|_{H}^{2} d s\right\} \leq C\left(M,\left\|u_{0}\right\|_{H}\right),
$$

where the constant $C$ is independent of $\varepsilon$ and $R$.

Using the same arguments as the proof of Theorem 5.2 in [8], for every $\eta>0$, it gives that

$$
\lim _{R \rightarrow+\infty} \sup _{\varepsilon>0} \int_{0}^{T}\left\|u_{h^{\varepsilon}}^{\eta, R}(t)-u_{h^{\varepsilon}}^{\eta}(t)\right\|_{H}^{2} d t=0 .
$$

With the aid of the above two approximation processes (4.43) and (4.44), it follows that for any $\varepsilon, \eta, R>0$,

$$
\begin{aligned}
& \left\|u_{h^{\varepsilon}}-u_{h}\right\|_{L^{1}\left([0, T] ; L^{1}\left(\mathbb{T}^{N}\right)\right)} \\
\leq & \left\|u_{h^{\varepsilon}}^{\eta}-u_{h^{\varepsilon}}\right\|_{L^{1}\left([0, T] ; L^{1}\left(\mathbb{T}^{N}\right)\right)}+\left\|u_{h^{\varepsilon}}^{\eta}-u_{h^{\varepsilon}}^{\eta, R}\right\|_{L^{1}\left([0, T] ; L^{1}\left(\mathbb{T}^{N}\right)\right)}+\left\|u_{h^{\varepsilon}}^{\eta, R}-u_{h}^{\eta, R}\right\|_{L^{1}\left([0, T] ; L^{1}\left(\mathbb{T}^{N}\right)\right)} \\
& +\left\|u_{h}^{\eta, R}-u_{h^{\eta}}^{\eta}\right\|_{L^{1}\left([0, T] ; L^{1}\left(\mathbb{T}^{N}\right)\right)}+\left\|u_{h}^{\eta}-u_{h}\right\|_{L^{1}\left([0, T] ; L^{1}\left(\mathbb{T}^{N}\right)\right)} .
\end{aligned}
$$

Thus, in order to establish the continuity of the skeleton equations, several steps are involved.

Firstly, we prove the compactness of $\left\{u_{h^{\varepsilon}}^{\eta, R}, \varepsilon>0\right\}$. For simplicity, we denote that $u_{\varepsilon}^{\eta, R}:=u_{h^{\varepsilon}}^{\eta, R}$.

As in [14], we introduce the following space. Let $K$ be a separable Banach space with the norm $\|\cdot\|_{K}$. Given $p>1, \alpha \in(0,1)$, let $W^{\alpha, p}([0, T] ; K)$ be the Sobolev space of all functions $u \in L^{p}([0, T] ; K)$ such that

$$
\int_{0}^{T} \int_{0}^{T} \frac{\|u(t)-u(s)\|_{K}^{p}}{|t-s|^{1+\alpha p}} d t d s<\infty
$$

which is then endowed with the norm

$$
\|u\|_{W^{\alpha, p}([0, T] ; K)}^{p}=\int_{0}^{T}\|u(t)\|_{K}^{p} d t+\int_{0}^{T} \int_{0}^{T} \frac{\|u(t)-u(s)\|_{K}^{p}}{|t-s|^{1+\alpha p}} d t d s .
$$

The following result can be found in [14].

Lemma 4.1. Let $B_{0} \subset B \subset B_{1}$ be three Banach spaces. Assume that both $B_{0}$ and $B_{1}$ are reflexive, and $B_{0}$ is compactly embedded in $B$. Let $p \in(1, \infty)$ and $\alpha \in(0,1)$ be given. Let $\Lambda$ be the space

$$
\Lambda:=L^{p}\left([0, T] ; B_{0}\right) \cap W^{\alpha, p}\left([0, T] ; B_{1}\right)
$$

endowed with the natural norm. Then the embedding of $\Lambda$ in $L^{p}([0, T] ; B)$ is compact. 
We then have the following result.

Proposition 4.4. For any $\eta, R>0,\left\{u_{\varepsilon}^{\eta, R}, \varepsilon>0\right\}$ is compact in $L^{2}([0, T] ; H)$.

Proof. From (4.44), $u_{\varepsilon}^{\eta, R}$ can be written as

$$
\begin{aligned}
u_{\varepsilon}^{\eta, R}(t) & =u_{0}+\eta \int_{0}^{t} \Delta u_{\varepsilon}^{\eta, R} d s-\int_{0}^{t} \operatorname{div}\left(A^{R}\left(u_{\varepsilon}^{\eta, R}(s)\right)\right) d s+\int_{0}^{t} \Phi\left(u_{\varepsilon}^{\eta, R}\right) h^{\varepsilon}(s) d s \\
& =: \quad I_{1}^{\varepsilon}+I_{2}^{\varepsilon}+I_{3}^{\varepsilon}+I_{4}^{\varepsilon} .
\end{aligned}
$$

Clearly, $\left\|I_{1}^{\varepsilon}\right\|_{H} \leq C_{1}$. Next,

$$
\begin{aligned}
\left\|-\Delta u_{\varepsilon}^{\eta, R}\right\|_{H^{-1}} & =\sup _{\|v\|_{H^{1}} \leq 1}\left|\left\langle v,-\Delta u_{\varepsilon}^{\eta, R}\right\rangle\right| \\
& =\sup _{\|v\|_{H^{1}} \leq 1}\left|\left\langle\nabla v, \nabla u_{\varepsilon}^{\eta, R}\right\rangle\right| \\
& \leq C\left\|\nabla u_{\varepsilon}^{\eta, R}\right\|_{H}
\end{aligned}
$$

which then yields the following

$$
\begin{aligned}
\left\|I_{2}^{\varepsilon}(t)-I_{2}^{\varepsilon}(s)\right\|_{H^{-1}}^{2} & =\eta\left\|\int_{s}^{t}-\Delta u_{\varepsilon}^{\eta, R}(l) d l\right\|_{H^{-1}}^{2} \\
& \leq C(t-s) \int_{s}^{t}\left\|-\Delta u_{\varepsilon}^{\eta, R}(l)\right\|_{H^{-1}}^{2} d l \\
& \leq C(t-s) \int_{s}^{t}\left\|\nabla u_{\varepsilon}^{\eta, R}(l)\right\|_{H}^{2} d l .
\end{aligned}
$$

Hence, by (4.45), we have for $\alpha \in\left(0, \frac{1}{2}\right)$,

$$
\begin{aligned}
& \sup _{\varepsilon}\left\|I_{2}^{\varepsilon}\right\|_{W^{\alpha, 2}\left([0, T] ; H^{-1}\left(\mathbb{T}^{N}\right)\right)}^{2} \\
\leq & \int_{0}^{T}\left\|I_{2}^{\varepsilon}(t)\right\|_{H^{-1}}^{2} d t+\int_{0}^{T} \int_{0}^{T} \frac{\left\|I_{2}^{\varepsilon}(t)-I_{2}^{\varepsilon}(s)\right\|_{H^{-1}}^{2}}{|t-s|^{1+2 \alpha}} d s d t \\
\leq & C_{2}(\alpha) .
\end{aligned}
$$

By integration by parts formula and the linear growth of $A^{R}$, we have

$$
\begin{aligned}
\left\|\operatorname{div}\left(A^{R}\left(u_{\varepsilon}^{\eta, R}(s)\right)\right)\right\|_{H^{-1}} & =\sup _{\|v\|_{H^{1}} \leq 1}\left|\left\langle v, \operatorname{div}\left(A^{R}\left(u_{\varepsilon}^{\eta, R}(s)\right)\right)\right\rangle\right| \\
& =\sup _{\|v\|_{H^{1}} \leq 1}\left|\left\langle\nabla v, A^{R}\left(u_{\varepsilon}^{\eta, R}(s)\right)\right\rangle\right| \\
& \leq C(R) \sup _{\|v\|_{H^{1}} \leq 1} \int_{\mathbb{T}^{N}}|\nabla v|\left(1+\left|u_{\varepsilon}^{\eta, R}(s)\right|\right) d x \\
& \leq C(R)\left(1+\left\|u_{\varepsilon}^{\eta, R}(s)\right\|_{H}^{2}\right)
\end{aligned}
$$


which gives that

$$
\begin{aligned}
\left\|I_{3}^{\varepsilon}(t)-I_{3}^{\varepsilon}(s)\right\|_{H^{-1}}^{2} & =\left\|\int_{s}^{t} \operatorname{div}\left(A^{R}\left(u_{\varepsilon}^{\eta, R}(l)\right)\right) d l\right\|_{H^{-1}}^{2} \\
& \leq C(R)(t-s) \int_{s}^{t}\left\|\operatorname{div}\left(A^{R}\left(u_{\varepsilon}^{\eta, R}(l)\right)\right)\right\|_{H^{-1}}^{2} d l \\
& \leq C(R)(t-s) \int_{s}^{t}\left(1+\left\|u_{\varepsilon}^{\eta, R}(l)\right\|_{H}^{2}\right) d l \\
& \leq C(R)(t-s)^{2}\left[1+\sup _{t \in[0, T]}\left\|u_{\varepsilon}^{\eta, R}(t)\right\|_{H}^{2}\right] .
\end{aligned}
$$

Hence, we deduce from (4.45) that for $\alpha \in\left(0, \frac{1}{2}\right)$,

$$
\begin{aligned}
& \sup _{\varepsilon}\left\|I_{3}^{\varepsilon}\right\|_{W^{\alpha, 2}\left([0, T] ; H^{-1}\left(\mathbb{T}^{N}\right)\right)}^{2} \\
\leq & \int_{0}^{T}\left\|I_{3}^{\varepsilon}(t)\right\|_{H^{-1}}^{2} d t+\int_{0}^{T} \int_{0}^{T} \frac{\left\|I_{3}^{\varepsilon}(t)-I_{3}^{\varepsilon}(s)\right\|_{H^{-1}}^{2}}{|t-s|^{1+2 \alpha}} d s d t \\
\leq & C_{3}(\alpha) .
\end{aligned}
$$

Moreover, by (2.14), it follows that

$$
\begin{aligned}
\left\|\Phi\left(u_{\varepsilon}^{\eta, R}\right) h^{\varepsilon}(l)\right\|_{H}^{2} & \leq\left\|\Phi\left(u_{\varepsilon}^{\eta, R}\right)\right\|_{\mathcal{L}_{2}(U, H)}^{2}\left|h^{\varepsilon}(l)\right|_{U}^{2} \\
& \leq D_{0}\left(1+\left\|u_{\varepsilon}^{\eta, R}\right\|_{H}^{2}\right)\left|h^{\varepsilon}(l)\right|_{U}^{2},
\end{aligned}
$$

then, by Hölder inequality, we get

$$
\begin{aligned}
\left\|I_{4}^{\varepsilon}(t)-I_{4}^{\varepsilon}(s)\right\|_{H}^{2} & =\left\|\int_{s}^{t} \Phi\left(u_{\varepsilon}^{\eta, R}\right) h^{\varepsilon}(l) d l\right\|_{H}^{2} \\
& \leq(t-s) \int_{s}^{t}\left\|\Phi\left(u_{\varepsilon}^{\eta, R}\right) h^{\varepsilon}(l)\right\|_{H}^{2} d l \\
& \leq D_{0}(t-s)\left(1+\sup _{t \in[0, T]}\left\|u_{\varepsilon}^{\eta, R}(t)\right\|_{H}^{2}\right) \int_{s}^{t}\left|h^{\varepsilon}(l)\right|_{U}^{2} d l \\
& \leq D_{0} M(t-s)\left(1+\sup _{t \in[0, T]}\left\|u_{\varepsilon}^{\eta, R}(t)\right\|_{H}^{2}\right) .
\end{aligned}
$$

Thus, we deduce from (4.45) that for $\alpha \in\left(0, \frac{1}{2}\right)$,

$$
\begin{aligned}
& \sup _{\varepsilon}\left\|I_{4}^{\varepsilon}\right\|_{W^{\alpha, 2}([0, T] ; H)}^{2} \\
\leq & \int_{0}^{T}\left\|I_{4}^{\varepsilon}(t)\right\|_{H}^{2} d t+\int_{0}^{T} \int_{0}^{T} \frac{\left\|I_{4}^{\varepsilon}(t)-I_{4}^{\varepsilon}(s)\right\|_{H}^{2}}{|t-s|^{1+2 \alpha}} d s d t \\
\leq & C_{4}(\alpha) .
\end{aligned}
$$

Collecting the above estimates, we conclude that for $\alpha \in\left(0, \frac{1}{2}\right)$,

$$
\sup _{\varepsilon}\left\|u_{\varepsilon}^{\eta, R}\right\|_{W^{\alpha, 2}\left([0, T] ; H^{-1}\left(\mathbb{T}^{N}\right)\right)}^{2} \leq C(\alpha) .
$$

Applying (4.45) and Lemma 4.1, we obtain the desired result. 
Furthermore, we apply the doubling of variables method to obtain the convergence of the sequence $\left\{u_{h}^{\eta}, \eta>0\right\}$ to $u_{h}$ in $L^{1}\left([0, T] ; L^{1}\left(\mathbb{T}^{N}\right)\right)$ uniformly on $h \in S_{M}$.

Proposition 4.5. We have

$$
\lim _{\eta \rightarrow 0} \sup _{h \in S_{M}}\left\|u_{h}^{\eta}-u_{h}\right\|_{L^{1}\left([0, T] ; L^{1}\left(\mathbb{T}^{N}\right)\right)}=0 .
$$

Proof. For any $h \in S_{M}$, we consider the kinetic solution $f_{1}(x, t, \xi)=I_{u_{h}(x, t)>\xi}$ of the skeleton equation (3.18) with the corresponding kinetic measure $m_{1}$. As the proof of (4.23), for $\varphi_{1} \in C_{c}^{\infty}\left(\mathbb{T}_{x}^{N} \times \mathbb{R}_{\xi}\right)$, we have

$$
\begin{aligned}
\left\langle f_{1}^{ \pm}(t), \varphi_{1}\right\rangle= & \left\langle f_{1,0}, \varphi_{1}\right\rangle+\int_{0}^{t}\left\langle f_{1}(s), a(\xi) \cdot \nabla_{x} \varphi_{1}(x, \xi)\right\rangle d s \\
& +\sum_{k \geq 1} \int_{0}^{t} \int_{\mathbb{T}^{N}} \int_{\mathbb{R}} \varphi_{1}(x, \xi) g_{k}(x, \xi) h^{k}(s) d v_{x, s}^{1}(\xi) d x d s-\left\langle m_{1}, \partial_{\xi} \varphi_{1}\right\rangle([0, t]),
\end{aligned}
$$

where $f_{1,0}=I_{u_{0}>\xi}$ and $v_{x, s}^{1}(\xi)=\partial_{\xi} \bar{f}_{1}^{ \pm}(s, x, \xi)=-\partial_{\xi} f_{1}^{ \pm}(s, x, \xi)=\delta_{u_{h}^{ \pm}=\xi}$. Similarly, consider the generalized kinetic solution $f_{2}^{\eta}(y, t, \zeta)=I_{u_{h}^{\eta}(y, t)>\zeta}$ of equation (4.43) with $h^{\varepsilon}$ replaced by $h$ and the corresponding kinetic measure is denoted by $m_{2}^{\eta}$. For $\varphi_{2} \in C_{c}^{\infty}\left(\mathbb{T}_{y}^{N} \times \mathbb{R}_{\zeta}\right)$, we have

$$
\begin{aligned}
\left\langle\bar{f}_{2}^{\eta, \pm}(t), \varphi_{2}\right\rangle= & \left\langle\bar{f}_{2,0}, \varphi_{2}\right\rangle+\int_{0}^{t}\left\langle\bar{f}_{2}^{\eta}(s), a(\zeta) \cdot \nabla_{y} \varphi_{2}(s)\right\rangle d s \\
& -\sum_{k \geq 1} \int_{0}^{t} \int_{\mathbb{T}^{N}} \int_{\mathbb{R}} g_{k}(y, \zeta) \varphi_{2}(x, \xi) h^{k}(s) d v_{y, s}^{2, \eta}(\zeta) d y d s+\left\langle m_{2}^{\eta}, \partial_{\zeta} \varphi_{2}\right\rangle([0, t]) \\
& -\eta \int_{0}^{t}\left\langle\bar{f}_{2}^{\eta, \pm}(s), \Delta_{y} \varphi_{2}(s)\right\rangle d s,
\end{aligned}
$$

where $f_{2,0}=I_{u_{0}>\zeta}$ and $v_{y, s}^{2, \eta}(\zeta)=-\partial_{\zeta} f_{2}^{\eta, \pm}(s, y, \zeta)=\partial_{\zeta} \bar{f}_{2}^{\eta, \pm}(s, y, \zeta)=\delta_{u_{h}^{\eta, \pm}=\zeta}$.

Setting $\alpha(x, \xi, y, \zeta)=\varphi_{1}(x, \xi) \varphi_{2}(y, \zeta)$, using integration by parts formula, we have

$$
\begin{aligned}
\left\langle\left\langle f_{1}^{ \pm}(t) \bar{f}_{2}^{\eta, \pm}(t), \alpha\right\rangle\right\rangle= & \left\langle\left\langle f_{1,0} \bar{f}_{2,0}, \alpha\right\rangle\right\rangle+\int_{0}^{t} \int_{\left(\mathbb{T}^{N}\right)^{2}} \int_{\mathbb{R}^{2}} f_{1} \bar{f}_{2}^{\eta}\left(a(\xi) \cdot \nabla_{x}+a(\zeta) \cdot \nabla_{y}\right) \alpha d \xi d \zeta d x d y d s \\
& -\sum_{k \geq 1} \int_{0}^{t} \int_{\left(\mathbb{T}^{N}\right)^{2}} \int_{\mathbb{R}^{2}} f_{1}^{ \pm}(s, x, \xi) \alpha g_{k}(y, \zeta) h^{k}(s) d \xi d v_{y, s}^{2, \eta}(\zeta) d x d y d s \\
& +\sum_{k \geq 1} \int_{0}^{t} \int_{\left(\mathbb{T}^{N}\right)^{2}} \int_{\mathbb{R}^{2}} \bar{f}_{2}^{\eta, \pm}(s, y, \zeta) \alpha g_{k}(x, \xi) h^{k}(s) d \zeta d v_{x, s}^{1}(\xi) d x d y d s \\
& +\int_{0}^{t} \int_{\left(\mathbb{T}^{N}\right)^{2}} \int_{\mathbb{R}^{2}} f_{1}^{ \pm}(s, x, \xi) \partial_{\zeta} \alpha d m_{2}^{\eta}(y, \zeta, s) d \xi d x \\
& -\int_{0}^{t} \int_{\left(\mathbb{T}^{N}\right)^{2}} \int_{\mathbb{R}^{2}} \bar{f}_{2}^{\eta, \pm}(s, y, \zeta) \partial_{\xi} \alpha d m_{1}(x, \xi, s) d \zeta d y \\
& -\eta \int_{0}^{t} \int_{\left(\mathbb{T}^{N}\right)^{2}} \int_{\mathbb{R}^{2}} f_{1}^{ \pm} \bar{f}_{2}^{\eta, \pm} \Delta_{y} \alpha d \xi d \zeta d x d y d s \\
:= & \left\langle\left\langle f_{1,0} \bar{f}_{2,0}, \alpha\right\rangle\right\rangle+R_{1}+R_{2}+R_{3}+R_{4}+R_{5}+R_{6} .
\end{aligned}
$$


Similarly, we get

$$
\begin{aligned}
\left\langle\left\langle\bar{f}_{1}^{ \pm}(t) f_{2}^{\eta, \pm}(t), \alpha\right\rangle\right\rangle= & \left\langle\left\langle\bar{f}_{1,0} f_{2,0}, \alpha\right\rangle\right\rangle+\int_{0}^{t} \int_{\left(\mathbb{T}^{N}\right)^{2}} \int_{\mathbb{R}^{2}} \bar{f}_{1} f_{2}^{\eta}\left(a(\xi) \cdot \nabla_{x}+a(\zeta) \cdot \nabla_{y}\right) \alpha d \xi d \zeta d x d y d s \\
& +\sum_{k \geq 1} \int_{0}^{t} \int_{\left(\mathbb{T}^{N}\right)^{2}} \int_{\mathbb{R}^{2}} \bar{f}_{1}^{ \pm}(s, x, \xi) \alpha g_{k}(y, \zeta) h^{k}(s) d \xi d v_{y, s}^{2, \eta}(\zeta) d x d y d s \\
& -\sum_{k \geq 1} \int_{0}^{t} \int_{\left(\mathbb{T}^{N}\right)^{2}} \int_{\mathbb{R}^{2}} f_{2}^{\eta, \pm}(s, y, \zeta) \alpha g_{k}(x, \xi) h^{k}(s) d \zeta d v_{x, s}^{1}(\xi) d x d y d s \\
& -\int_{0}^{t} \int_{\left(\mathbb{T}^{N}\right)^{2}} \int_{\mathbb{R}^{2}} \bar{f}_{1}^{ \pm}(s, x, \xi) \partial_{\zeta} \alpha d m_{2}^{\eta}(y, \zeta, s) d \xi d x \\
& +\int_{0}^{t} \int_{\left(\mathbb{T}^{N}\right)^{2}} \int_{\mathbb{R}^{2}} f_{2}^{\eta, \pm}(s, y, \zeta) \partial_{\xi} \alpha d m_{1}(x, \xi, s) d \zeta d y \\
& +\eta \int_{0}^{t} \int_{\left(\mathbb{T}^{N}\right)^{2}} \int_{\mathbb{R}^{2}} \bar{f}_{1}^{ \pm} f_{2}^{\eta, \pm} \Delta_{y} \alpha d \xi d \zeta d x d y d s \\
:= & \left\langle\left\langle\bar{f}_{1,0} f_{2,0}, \alpha\right\rangle\right\rangle+\bar{R}_{1}+\bar{R}_{2}+\bar{R}_{3}+\bar{R}_{4}+\bar{R}_{5}+\bar{R}_{6} .
\end{aligned}
$$

As the proof of Proposition 4.2 and Theorem 4.3, taking $\alpha=\rho_{\gamma}(x-y) \psi_{\delta}(\xi-\zeta)$, where $\rho_{\gamma}$ and $\psi_{\delta}$ are approximations to the identity on $\mathbb{T}^{N}$ and $\mathbb{R}$, respectively, we have

$$
\begin{aligned}
& \int_{\left(\mathbb{T}^{N}\right)^{2}} \int_{\mathbb{R}^{2}} \rho_{\gamma}(x-y) \psi_{\delta}(\xi-\zeta)\left(f_{1}^{ \pm}(x, t, \xi) \bar{f}_{2}^{\eta, \pm}(y, t, \zeta)+\bar{f}_{1}^{ \pm}(x, t, \xi) f_{2}^{\eta, \pm}(y, t, \zeta)\right) d \xi d \zeta d x d y \\
\leq & \int_{\left(\mathbb{T}^{N}\right)^{2}} \int_{\mathbb{R}^{2}} \rho_{\gamma}(x-y) \psi_{\delta}(\xi-\zeta)\left(f_{1,0}(x, \xi) \bar{f}_{2,0}(y, \zeta)+\bar{f}_{1,0}(x, \xi) f_{2,0}(y, \zeta)\right) d \xi d \zeta d x d y \\
& +\sum_{i=1}^{6}\left(\tilde{R}_{i}+\tilde{\bar{R}}_{i}\right) .
\end{aligned}
$$

where $\tilde{R}_{i}, \tilde{\bar{R}}_{i}$ in (4.49) are the corresponding $R_{i}, \bar{R}_{i}$ with $\alpha=\rho_{\gamma}(x-y) \psi_{\delta}(\xi-\zeta)$ for $i=1, \cdots, 6$.

Referring to Proposition 13 in [9], $\tilde{R}_{4}, \tilde{R}_{5}, \tilde{\bar{R}}_{4}, \tilde{\bar{R}}_{5}$ are both non-positive. From (4.28), $\tilde{R}_{1}$ and $\tilde{\bar{R}}_{1}$ can be written as

$$
\begin{aligned}
& \tilde{R}_{1}=\int_{0}^{t} \int_{\left(\mathbb{T}^{N}\right)^{2}} \int_{\mathbb{R}^{2}} f_{1} \bar{f}_{2}^{\eta}(a(\xi)-a(\zeta)) \cdot \nabla_{x} \rho_{\gamma}(x-y) \psi_{\delta}(\xi-\zeta) d \xi d \zeta d x d y d s, \\
& \tilde{\bar{R}}_{1}=\int_{0}^{t} \int_{\left(\mathbb{T}^{N}\right)^{2}} \int_{\mathbb{R}^{2}} \bar{f}_{1} f_{2}^{\eta}(a(\xi)-a(\zeta)) \cdot \nabla_{x} \rho_{\gamma}(x-y) \psi_{\delta}(\xi-\zeta) d \xi d \zeta d x d y d s .
\end{aligned}
$$

Similarly to the proof of Theorem 15 in [9], we get

$$
\left|\tilde{R}_{1}\right| \leq T C_{p} \delta \gamma^{-1}, \quad\left|\tilde{\bar{R}}_{1}\right| \leq T C_{p} \delta \gamma^{-1}
$$

Moreover, with the aid of $\gamma_{1}(\xi, \zeta)=\int_{-\infty}^{\xi} \psi_{\delta}\left(\xi^{\prime}-\zeta\right) d \xi^{\prime}, \gamma_{2}(\xi, \zeta)=\int_{\zeta}^{\infty} \psi_{\delta}\left(\xi-\zeta^{\prime}\right) d \zeta^{\prime}$ and $\gamma_{1}(\xi, \zeta)=$ 
$\gamma_{2}(\xi, \zeta)$, by the same arguments as the proof of Theorem 4.3, it follows that

$$
\begin{aligned}
\tilde{\tilde{R}}_{2}+\tilde{\bar{R}}_{3} & =\tilde{R}_{2}+\tilde{R}_{3} \\
& =\sum_{k \geq 1} \int_{0}^{t} \int_{\left(\mathbb{T}^{N}\right)^{2}} \rho_{\gamma}(x-y) \int_{\mathbb{R}^{2}} \gamma_{1}(\xi, \zeta)\left(g_{k}(x, \xi)-g_{k}(y, \zeta)\right) h^{k}(s) d v_{x, s}^{1} \otimes d v_{y, s}^{2, \eta}(\xi, \zeta) d x d y d s \\
& \leq \int_{0}^{t}|h(s)|_{U} \int_{\left(\mathbb{T}^{N}\right)^{2}} \rho_{\gamma}(x-y) \int_{\mathbb{R}^{2}} \gamma_{1}(\xi, \zeta)\left(\sum_{k \geq 1}\left|g_{k}(x, \xi)-g_{k}(y, \zeta)\right|^{2}\right)^{\frac{1}{2}} d v_{x, s}^{1} \otimes d v_{y, s}^{2, \eta}(\xi, \zeta) d x d y d s .
\end{aligned}
$$

Applying the same method as the estimate of $\tilde{K}_{2}$ in Theorem 4.3, we deduce that

$$
\begin{aligned}
\tilde{\bar{R}}_{2}+\tilde{\bar{R}}_{3}= & \tilde{R}_{2}+\tilde{R}_{3} \\
\leq & \sqrt{D_{1}} \int_{0}^{t}|h(s)|_{U} \int_{\left(\mathbb{T}^{N}\right)^{2}} \rho_{\gamma}(x-y) \int_{\mathbb{R}^{2}} \gamma_{1}(\xi, \zeta)|x-y| d v_{x, s}^{1} \otimes d v_{y, s}^{2, \eta}(\xi, \zeta) d x d y d s \\
& +\sqrt{D_{1}} \int_{0}^{t}|h(s)|_{U} \int_{\left(\mathbb{T}^{N}\right)^{2}} \rho_{\gamma}(x-y) \int_{\mathbb{R}^{2}} \gamma_{1}(\xi, \zeta)|\xi-\zeta| d v_{x, s}^{1} \otimes d v_{y, s}^{2, \eta}(\xi, \zeta) d x d y d s \\
\leq & (\gamma+2 \delta) \sqrt{D_{1}}\left(T+\int_{0}^{T}|h(s)|_{U}^{2} d s\right) \\
& +\sqrt{D_{1}} \int_{0}^{t}|h(s)|_{U} \int_{\left(\mathbb{T}^{N}\right)^{2}} \int_{\mathbb{R}^{2}}\left(f_{1}^{ \pm} \bar{f}_{2}^{\eta, \pm}+\bar{f}_{1}^{ \pm} f_{2}^{\eta, \pm}\right) \rho_{\gamma}(x-y) \psi_{\delta}(\xi-\zeta) d x d y d \xi d \zeta d s .
\end{aligned}
$$

For the term $\tilde{R}_{6}$, it can be estimated as follows:

$$
\begin{aligned}
\tilde{R}_{6} & \leq \eta \int_{0}^{t} \int_{\left(\mathbb{T}^{N}\right)^{2}} \int_{\mathbb{R}^{2}} f_{1}^{ \pm} \bar{f}_{2}^{\eta, \pm} \Delta_{y} \rho_{\gamma}(x-y) \psi_{\delta}(\xi-\zeta) d \xi d \zeta d x d y d s \\
& =\eta \int_{0}^{t} \int_{\left.\mathbb{T}^{N}\right)^{2}} \Delta_{x} \rho_{\gamma}(x-y)\left[\int_{\mathbb{R}^{2}} f_{1}^{ \pm} \bar{f}_{2}^{\eta, \pm} \psi_{\delta}(\xi-\zeta) d \xi d \zeta\right] d x d y d s \\
& =\eta \int_{0}^{t} \int_{\left(\mathbb{T}^{N}\right)^{2}} \Delta_{x} \rho_{\gamma}(x-y)\left[\int_{\mathbb{R}^{2}} l(\xi, \zeta) d v_{x, s}^{1} \otimes d v_{y, s}^{2, \eta}(\xi, \zeta)\right] d x d y d s,
\end{aligned}
$$

where

$$
l(\xi, \zeta)=\int_{\zeta}^{\infty} \int_{-\infty}^{\xi} \psi_{\delta}\left(\xi^{\prime}-\zeta^{\prime}\right) d \xi^{\prime} d \zeta^{\prime}
$$

Moreover, let $\xi^{\prime \prime}=\xi^{\prime}-\zeta^{\prime}$, it follows that

$$
\begin{aligned}
l(\xi, \zeta) & \leq \int_{\zeta}^{\infty}\left(\int_{\left\{\left|\xi^{\prime \prime}\right|<\delta, \xi^{\prime \prime}<\xi-\zeta^{\prime}\right\}} \psi_{\delta}\left(\xi^{\prime \prime}\right) d \xi^{\prime \prime}\right) d \zeta^{\prime} \\
& \leq C \int_{\zeta}^{\xi+\delta} \delta\left\|\psi_{\delta}\right\|_{L^{\infty}} d \zeta^{\prime} \\
& \leq C(|\xi|+|\zeta|+\delta) .
\end{aligned}
$$

Then,

$$
\int_{\mathbb{R}^{2}} l(\xi, \zeta) d v_{x, s}^{1} \otimes d v_{y, s}^{2, \eta}(\xi, \zeta) \leq C(1+\delta),
$$


where we have used the property that the measures $v_{x, s}^{1, \eta}$ and $v_{y, s}^{2}$ vanish at the infinity. Thus, we have

$$
\tilde{R}_{6} \leq C(1+\delta) \eta \int_{0}^{t} \int_{\left(\mathbb{T}^{N}\right)^{2}} \Delta_{y} \rho_{\gamma}(x-y) d x d y d s \leq C(1+\delta) \eta T \gamma^{-2}
$$

Similarly, using the same method as above, we conclude that $\tilde{\bar{R}}_{6}$ has the same estimate of $\tilde{R}_{6}$.

Based on all the above estimates, it follows that

$$
\begin{aligned}
& \int_{\left(\mathbb{T}^{N}\right)^{2}} \int_{\mathbb{R}^{2}} \rho_{\gamma}(x-y) \psi_{\delta}(\xi-\zeta)\left(f_{1}^{ \pm}(x, t, \xi) \bar{f}_{2}^{\eta, \pm}(y, t, \zeta)+\bar{f}_{1}^{ \pm}(x, t, \xi) f_{2}^{\eta, \pm}(y, t, \zeta)\right) d \xi d \zeta d x d y \\
\leq & \int_{\left(\mathbb{T}^{N}\right)^{2}} \int_{\mathbb{R}^{2}} \rho_{\gamma}(x-y) \psi_{\delta}(\xi-\zeta)\left(f_{1,0}(x, \xi) \bar{f}_{2,0}(y, \zeta)+\bar{f}_{1,0}(x, \xi) f_{2,0}(y, \zeta)\right) d \xi d \zeta d x d y \\
& +2 T C_{p} \delta \gamma^{-1}+2 C(1+\delta) \eta T \gamma^{-2}+2(\gamma+2 \delta) \sqrt{D_{1}}\left(T+\int_{0}^{T}|h(s)|_{U}^{2} d s\right) \\
& +2 \sqrt{D_{1}} \int_{0}^{t}|h(s)|_{U} \int_{\left(\mathbb{T}^{N}\right)^{2}} \int_{\mathbb{R}^{2}}\left(f_{1}^{ \pm} \bar{f}_{2}^{\eta, \pm}+\bar{f}_{1}^{ \pm} f_{2}^{\eta, \pm}\right) \rho_{\gamma}(x-y) \psi_{\delta}(\xi-\zeta) d x d y d \xi d \zeta d s
\end{aligned}
$$

By Gronwall inequality, we get

$$
\begin{aligned}
& \int_{\left(\mathbb{T}^{N}\right)^{2}} \int_{\mathbb{R}^{2}} \rho_{\gamma}(x-y) \psi_{\delta}(\xi-\zeta)\left(f_{1}^{ \pm}(x, t, \xi) \bar{f}_{2}^{\eta, \pm}(y, t, \zeta)+\bar{f}_{1}^{ \pm}(x, t, \xi) f_{2}^{\eta, \pm}(y, t, \zeta)\right) d \xi d \zeta d x d y \\
& \leq e^{2 \sqrt{D_{1}}\left(T+\int_{0}^{T}|h(s)|_{U}^{2} d s\right)}\left[\int_{\left(\mathbb{T}^{N}\right)^{2}} \int_{\mathbb{R}^{2}} \rho_{\gamma}(x-y) \psi_{\delta}(\xi-\zeta)\left(f_{1,0}(x, \xi) \bar{f}_{2,0}(y, \zeta)+\bar{f}_{1,0}(x, \xi) f_{2,0}(y, \zeta)\right) d \xi d \zeta d x d y\right] \\
& +2 e^{2 \sqrt{D_{1}}\left(T+\int_{0}^{T}|h(s)|_{U}^{2} d s\right)}\left[T C_{p} \delta \gamma^{-1}+C(1+\delta) \eta T \gamma^{-2}+(\gamma+2 \delta) \sqrt{D_{1}}\left(T+\int_{0}^{T}|h(s)|_{U}^{2} d s\right)\right] \\
& \leq e^{2 \sqrt{D_{1}}\left(T+\int_{0}^{T}|h(s)|_{U}^{2} d s\right)}\left[\int_{\mathbb{T}^{N}} \int_{\mathbb{R}}\left(f_{1,0}(x, \xi) \bar{f}_{2,0}(x, \xi)+\bar{f}_{1,0}(x, \xi) f_{2,0}(x, \xi)\right) d x d \xi+\mathcal{E}_{0}(\gamma, \delta)\right] \\
& +2 e^{2 \sqrt{D_{1}}\left(T+\int_{0}^{T}|h(s)|_{U}^{2} d s\right)}\left[T C_{p} \delta \gamma^{-1}+C(1+\delta) \eta T \gamma^{-2}+(\gamma+2 \delta) \sqrt{D_{1}}\left(T+\int_{0}^{T}|h(s)|_{U}^{2} d s\right)\right],
\end{aligned}
$$

where $\mathcal{E}_{0}(\gamma, \delta)$ is independent of $\eta$ and converges to 0 as $\gamma, \delta \rightarrow 0$.

Let

$$
\begin{aligned}
\mathcal{E}_{t}(\eta, \gamma, \delta):= & \int_{\mathbb{T}^{N}} \int_{\mathbb{R}}\left(f_{1}^{ \pm}(x, t, \xi) \bar{f}_{2}^{\eta, \pm}(x, t, \xi)+\bar{f}_{1}^{ \pm}(x, t, \xi) f_{2}^{\eta, \pm}(x, t, \xi)\right) d x d \xi \\
& -\int_{\left(\mathbb{T}^{N}\right)^{2}} \int_{\mathbb{R}^{2}} \rho_{\gamma}(x-y) \psi_{\delta}(\xi-\zeta)\left(f_{1}^{ \pm}(x, t, \xi) \bar{f}_{2}^{\eta, \pm}(y, t, \zeta)+\bar{f}_{1}^{ \pm}(x, t, \xi) f_{2}^{\eta, \pm}(y, t, \zeta)\right) d \xi d \zeta d x d y,
\end{aligned}
$$

we claim that $\mathcal{E}_{t}(\eta, \gamma, \delta)$ is independent of $\eta$. Indeed, 


$$
\begin{aligned}
\mathcal{E}_{t}(\eta, \gamma, \delta)= & {\left[\int_{\mathbb{T}^{N}} \int_{\mathbb{R}}\left(f_{1}^{ \pm}(x, t, \xi) \bar{f}_{2}^{\eta, \pm}(x, t, \xi)+\bar{f}_{1}^{ \pm}(x, t, \xi) f_{2}^{\eta, \pm}(x, t, \xi)\right) d x d \xi\right.} \\
& \left.-\int_{\left(\mathbb{T}^{N}\right)^{2}} \int_{\mathbb{R}} \rho_{\gamma}(x-y)\left(f_{1}^{ \pm}(x, t, \xi) \bar{f}_{2}^{\eta, \pm}(y, t, \xi)+\bar{f}_{1}^{ \pm}(x, t, \xi) f_{2}^{\eta, \pm}(y, t, \xi)\right) d \xi d x d y\right] \\
& +\left[\int_{\left(\mathbb{T}^{N}\right)^{2}} \int_{\mathbb{R}} \rho_{\gamma}(x-y)\left(f_{1}^{ \pm}(x, t, \xi) \bar{f}_{2}^{\eta, \pm}(y, t, \xi)+\bar{f}_{1}^{ \pm}(x, t, \xi) f_{2}^{\eta, \pm}(y, t, \xi)\right) d \xi d x d y\right. \\
& \left.-\int_{\left(\mathbb{T}^{N}\right)^{2}} \int_{\mathbb{R}^{2}} \rho_{\gamma}(x-y) \psi_{\delta}(\xi-\zeta)\left(f_{1}^{ \pm}(x, t, \xi) \hat{f}_{2}^{\eta, \pm}(y, t, \zeta)+\bar{f}_{1}^{ \pm}(x, t, \xi) f_{2}^{\eta, \pm}(y, t, \zeta)\right) d \xi d \zeta d x d y\right] \\
=: & H_{1}+H_{2},
\end{aligned}
$$

Applying the same method as (4.34) and (4.35), it follows that

$$
\left|H_{2}\right| \leq 2 \delta
$$

and

$$
\begin{aligned}
\left|H_{1}\right| \leq & \left|\int_{\left(\mathbb{T}^{N}\right)^{2}} \rho_{\gamma}(x-y) \int_{\mathbb{R}} I_{u_{h}^{ \pm}(x, t)>\xi}\left(I_{u_{h}^{\eta, \pm}(x, t) \leq \xi}-I_{u_{h}^{\eta, \pm}(y, t) \leq \xi}\right) d \xi d x d y\right| \\
& +\left|\int_{\left(\mathbb{T}^{N}\right)^{2}} \rho_{\gamma}(x-y) \int_{\mathbb{R}} I_{u_{h}^{ \pm}(x, t) \leq \xi}\left(I_{u_{h}^{\eta, \pm}(x, t)>\xi}-I_{u_{h}^{\eta, \pm}(y, t)>\xi}\right) d \xi d x d y\right| \\
\leq & 2 \int_{\left(\mathbb{T}^{N}\right)^{2}} \rho_{\gamma}(x-y)\left|u_{h}^{\eta, \pm}(x, t)-u_{h}^{\eta, \pm}(y, t)\right| d x d y .
\end{aligned}
$$

Combing (4.51) and (4.52), it yields

$$
\begin{aligned}
& \mathcal{E}_{t}(\eta, \gamma, \delta) \\
\leq & 2 \delta+2 \int_{\left(\mathbb{T}^{N}\right)^{2}} \rho_{\gamma}(x-y)\left|u_{h}^{\eta, \pm}(x, t)-u_{h}^{\eta, \pm}(y, t)\right| d x d y \\
= & 2 \delta+2 \int_{\left(\mathbb{T}^{N}\right)^{2}} \int_{\mathbb{R}^{2}} \rho_{\gamma}(x-y)\left(f_{2}^{\eta, \pm}(x, t, \xi) \bar{f}_{2}^{\eta, \pm}(y, t, \xi)+\bar{f}_{2}^{\eta, \pm}(x, t, \xi) f_{2}^{\eta, \pm}(y, t, \xi)\right) d \xi d x d y \\
\leq & 4 \delta+2 \int_{\left(\mathbb{T}^{N}\right)^{2}} \int_{\mathbb{R}^{2}} \rho_{\gamma}(x-y) \psi_{\delta}(\xi-\zeta)\left(f_{2}^{\eta, \pm}(x, t, \xi) f_{2}^{\eta, \pm}(y, t, \zeta)\right. \\
& \left.+f_{2}^{\eta, \pm}(x, t, \xi) f_{2}^{\eta, \pm}(y, t, \zeta)\right) d \xi d \zeta d x d y .
\end{aligned}
$$

Utilizing (4.48) and applying the similar method as the proof of (4.50), we obtain

$$
\begin{aligned}
& \int_{\left(\mathbb{T}^{N}\right)^{2}} \int_{\mathbb{R}^{2}} \rho_{\gamma}(x-y) \psi_{\delta}(\xi-\zeta)\left(f_{2}^{\eta, \pm}(x, t, \xi) \bar{f}_{2}^{\eta, \pm}(y, t, \zeta)+\bar{f}_{2}^{\eta, \pm}(x, t, \xi) f_{2}^{\eta, \pm}(y, t, \zeta)\right) d \xi d \zeta d x d y \\
\leq & e^{\sqrt{D_{1}}\left(T+\int_{0}^{T}|h(s)|_{U}^{2} d s\right)}\left[\int_{\mathbb{T}^{N}} \int_{\mathbb{R}}\left(f_{2,0} \bar{f}_{2,0}+\bar{f}_{2,0} f_{2,0}\right) d x d \xi+\mathcal{E}_{0}(\gamma, \delta)+J^{\sharp}\right] \\
& +2 e^{\sqrt{D_{1}}\left(T+\int_{0}^{T}|h(s)|_{U}^{2} d s\right)}\left[T C_{p} \delta \gamma^{-1}+(\gamma+2 \delta) \sqrt{D_{1}}\left(T+\int_{0}^{T}|h(s)|_{U}^{2} d s\right)\right],
\end{aligned}
$$


where $\mathcal{E}_{0}(\gamma, \delta)$ is different from that in (4.50) but they both converge to 0 , so we do not distinguish them. Moreover, by utilizing the property $\partial_{x} \alpha+\partial_{y} \alpha=0$, we deduce that

$$
\begin{aligned}
J^{\sharp}= & -\eta \int_{0}^{t} \int_{\left(\mathbb{T}^{N}\right)^{2}} \int_{\mathbb{R}^{2}} f_{2}^{\eta, \pm}(x, s, \xi) \hat{f}_{2}^{\eta, \pm}(y, s, \zeta) \Delta_{y} \alpha d \xi d \zeta d x d y d s \\
& +\eta \int_{0}^{t} \int_{\left(\mathbb{T}^{N}\right)^{2}} \int_{\mathbb{R}^{2}} f_{2}^{\eta, \pm}(x, s, \xi) f_{2}^{\eta, \pm}(y, s, \zeta) \Delta_{x} \alpha d \xi d \zeta d x d y d s \\
& -\eta \int_{0}^{t} \int_{\left(\mathbb{T}^{N}\right)^{2}} \int_{\mathbb{R}^{2}} f_{2}^{\eta, \pm}(x, s, \xi) f_{2}^{\eta, \pm}(y, s, \zeta) \Delta_{x} \alpha d \xi d \zeta d x d y d s \\
& +\eta \int_{0}^{t} \int_{\left(\mathbb{T}^{N}\right)^{2}} \int_{\mathbb{R}^{2}} f_{2}^{\eta, \pm}(x, s, \xi) f_{2}^{\eta, \pm}(y, s, \zeta) \Delta_{y} \alpha d \xi d \zeta d x d y d s \\
= & 0 .
\end{aligned}
$$

Hence,

$$
\begin{aligned}
& \int_{\left(\mathbb{T}^{N}\right)^{2}} \int_{\mathbb{R}^{2}} \rho_{\gamma}(x-y) \psi_{\delta}(\xi-\zeta)\left(f_{2}^{\eta, \pm}(x, t, \xi) f_{2}^{\eta, \pm}(y, t, \zeta)+f_{2}^{\eta, \pm}(x, t, \xi) f_{2}^{\eta, \pm}(y, t, \zeta)\right) d \xi d \zeta d x d y \\
\leq & e^{2 \sqrt{D_{1}}\left(T+\int_{0}^{T}|h(s)|_{U}^{2} d s\right)}\left[\mathcal{E}_{0}(\gamma, \delta)+2 T C_{p} \delta \gamma^{-1}+2(\gamma+2 \delta) \sqrt{D_{1}}\left(T+\int_{0}^{T}|h(s)|_{U}^{2} d s\right)\right], \quad
\end{aligned}
$$

Combing (4.53) and (4.54), we conclude that

$\mathcal{E}_{t}(\eta, \gamma, \delta)$

$$
\leq 4 \delta+2 e^{2 \sqrt{D_{1}}\left(T+\int_{0}^{T}|h(s)|_{U}^{2} d s\right)}\left[\mathcal{E}_{0}(\gamma, \delta)+2 T C_{p} \delta \gamma^{-1}+2(\gamma+2 \delta) \sqrt{D_{1}}\left(T+\int_{0}^{T}|h(s)|_{U}^{2} d s\right)\right],
$$

which implies that $\mathcal{E}_{t}(\eta, \gamma, \delta)$ is independent of $\eta$, so we denote that $\mathcal{E}_{t}(\gamma, \delta):=\mathcal{E}_{t}(\eta, \gamma, \delta)$.

From (4.50) and (4.55), we deduce that

$$
\begin{aligned}
& \int_{\mathbb{T}^{N}} \int_{\mathbb{R}}\left(f_{1}^{ \pm}(x, t, \xi) \bar{f}_{2}^{\eta, \pm}(x, t, \xi)+\bar{f}_{1}^{ \pm}(x, t, \xi) f_{2}^{\eta, \pm}(x, t, \xi)\right) d x d \xi \\
\leq & \int_{\left(\mathbb{T}^{N}\right)^{2}} \int_{\mathbb{R}^{2}} \rho_{\gamma}(x-y) \psi_{\delta}(\xi-\zeta)\left(f_{1}^{ \pm}(x, t, \xi) \bar{f}_{2}^{\eta, \pm}(y, t, \zeta)+\bar{f}_{1}^{ \pm}(x, t, \xi) f_{2}^{\eta, \pm}(y, t, \zeta)\right) d \xi d \zeta d x d y+\mathcal{E}_{t}(\gamma, \delta) \\
\leq & e^{2 \sqrt{D_{1}}\left(T+\int_{0}^{T}|h(s)|_{U}^{2} d s\right)}\left[\int_{\mathbb{T}^{N}} \int_{\mathbb{R}}\left(f_{1,0} \bar{f}_{2,0}+\bar{f}_{1,0} f_{2,0}\right) d x d \xi+\mathcal{E}_{0}(\gamma, \delta)+2 T C_{p} \delta \gamma^{-1}\right]+\mathcal{E}_{t}(\gamma, \delta) \\
& +2 e^{2 \sqrt{D_{1}}\left(T+\int_{0}^{T}|h(s)|_{U}^{2} d s\right)}\left[C(1+\delta) \eta T \gamma^{-2}+(\gamma+2 \delta) \sqrt{D_{1}}\left(T+\int_{0}^{T}|h(s)|_{U}^{2} d s\right)\right] \\
\leq & e^{2 \sqrt{D_{1}}\left(T+\int_{0}^{T}|h(s)|_{U}^{2} d s\right)}\left[3 \mathcal{E}_{0}(\gamma, \delta)+6 T C_{p} \delta \gamma^{-1}\right]+4 \delta \\
& +2 e^{2 \sqrt{D_{1}}\left(T+\int_{0}^{T}|h(s)|_{U}^{2} d s\right)}\left[C(1+\delta) \eta T \gamma^{-2}+3(\gamma+2 \delta) \sqrt{D_{1}}\left(T+\int_{0}^{T}|h(s)|_{U}^{2} d s\right)\right] .
\end{aligned}
$$

Then, we reach

$$
\begin{aligned}
& \sup _{h \in S_{M}} \int_{\mathbb{T}^{N}} \int_{\mathbb{R}}\left(f_{1}^{ \pm}(x, t, \xi) f_{2}^{\eta, \pm}(x, t, \xi)+\bar{f}_{1}^{ \pm}(x, t, \xi) f_{2}^{\eta, \pm}(x, t, \xi)\right) d x d \xi \\
\leq & e^{2 \sqrt{D_{1}}(T+M)}\left[3 \mathcal{E}_{0}(\gamma, \delta)+6 T C_{p} \delta \gamma^{-1}\right]+4 \delta \\
& +2 e^{2 \sqrt{D_{1}}(T+M)}\left[C(1+\delta) \eta T \gamma^{-2}+3(\gamma+2 \delta) \sqrt{D_{1}}(T+M)\right] .
\end{aligned}
$$


Taking $\delta=\gamma^{\frac{4}{3}}$ and $\gamma=\eta^{\frac{1}{3}}$, we get

$$
\begin{aligned}
& \sup _{h \in S_{M}} \int_{\mathbb{T}^{N}} \int_{\mathbb{R}}\left(f_{1}^{ \pm}(x, t, \xi) \bar{f}_{2}^{\eta, \pm}(x, t, \xi)+\bar{f}_{1}^{ \pm}(x, t, \xi) f_{2}^{\eta, \pm}(x, t, \xi)\right) d x d \xi \\
\leq & e^{2 \sqrt{D_{1}}(T+M)}\left[3 \mathcal{E}_{0}(\gamma, \delta)+6 T C_{p} \eta^{\frac{1}{9}}\right]+4 \eta^{\frac{4}{9}} \\
& +2 e^{2 \sqrt{D_{1}}(T+M)}\left[C T\left(1+\eta^{\frac{4}{9}}\right) \eta^{\frac{1}{3}}+3\left(\eta^{\frac{1}{3}}+2 \eta^{\frac{4}{9}}\right) \sqrt{D_{1}}(T+M)\right] .
\end{aligned}
$$

We deduce further from the following identities

$$
\int_{\mathbb{R}} I_{u_{h}>\xi} \overline{I_{u_{h}^{\eta}>\xi}^{\eta}} d \xi=\left(u_{h}-u_{h}^{\eta}\right)^{+}, \quad \int_{\mathbb{R}} \overline{I_{u_{h}>\xi}} I_{u_{h}^{\eta}>\xi} d \xi=\left(u_{h}-u_{h}^{\eta}\right)^{-},
$$

that

$$
\begin{aligned}
& \sup _{h \in S_{M}}\left\|u_{h}^{\eta}(t)-u_{h}(t)\right\|_{L^{1}\left(\mathbb{T}^{N}\right)} \\
\leq & e^{2 \sqrt{D_{1}}(T+M)}\left[3 \mathcal{E}_{0}(\gamma, \delta)+2 T C_{p} \eta^{\frac{1}{9}}+6 C T\left(1+\eta^{\frac{4}{9}}\right) \eta^{\frac{1}{3}}\right]+4 \eta^{\frac{4}{9}} \\
& +6 e^{2 \sqrt{D_{1}}(T+M)}\left(\eta^{\frac{1}{3}}+2 \eta^{\frac{4}{9}}\right) \sqrt{D_{1}}(T+M) .
\end{aligned}
$$

Therefore, we get

$$
\lim _{\eta \rightarrow 0} \sup _{h \in S_{M}}\left\|u_{h}^{\eta}(t)-u_{h}(t)\right\|_{L^{1}\left(\mathbb{T}^{N}\right)}=0
$$

We complete the proof.

Now, we are in a position to prove the continuity of $\mathcal{G}^{0}$.

Theorem 4.6. Assume $h^{\varepsilon} \rightarrow h$ weakly in $L^{2}([0, T] ; U)$. Then $u_{h^{\varepsilon}}$ converges to $u_{h}$ in $L^{1}\left([0, T] ; L^{1}\left(\mathbb{T}^{N}\right)\right)$, where $u_{h^{\varepsilon}}$ is the kinetic solution of (4.24) with $h$ replaced by $h^{\varepsilon}$.

Proof. Fix any $\eta, R>0$. For the solution $u_{h^{\varepsilon}}^{\eta, R}$ of (4.44), we shall firstly prove that when $h^{\varepsilon} \rightarrow h$ weakly in $L^{2}([0, T] ; U)$, we have $\lim _{\mathcal{E} \rightarrow 0}\left\|u_{h^{\varepsilon}}^{\eta, R}-u_{h}^{\eta, R}\right\|_{L^{1}\left([0, T] ; L^{1}\left(\mathbb{T}^{N}\right)\right)}=0$, where $u_{h}^{\eta, R}$ is the solution of (4.44) with $h^{\varepsilon}$ replaced by $h$.

In fact, by the chain rule, we have

$$
\begin{aligned}
& \left\|u_{h^{\varepsilon}}^{\eta, R}(t)-u_{h}^{\eta, R}(t)\right\|_{H}^{2}+2 \eta \int_{0}^{t}\left\|\nabla\left(u_{h^{\varepsilon}}^{\eta, R}-u_{h}^{\eta, R}\right)\right\|_{H}^{2} d s \\
\leq & 2 \int_{0}^{t}\left\langle A^{R}\left(u_{h^{\varepsilon}}^{\eta, R}\right)-A^{R}\left(u_{h}^{\eta, R}\right), \nabla\left(u_{h^{\varepsilon}}^{\eta, R}-u_{h}^{\eta, R}\right)\right\rangle d s+2 \int_{0}^{t}\left\langle\Phi\left(u_{h^{\varepsilon}}^{\eta, R}\right) h^{\varepsilon}(s)-\Phi\left(u_{h}^{\eta, R}\right) h(s), u_{h^{\varepsilon}}^{\eta, R}-u_{h}^{\eta, R}\right\rangle d s \\
\leq & 2 \int_{0}^{t}\left\langle A^{R}\left(u_{h^{\varepsilon}}^{\eta, R}\right)-A^{R}\left(u_{h}^{\eta, R}\right), \nabla\left(u_{h^{\varepsilon}}^{\eta, R}-u_{h}^{\eta, R}\right)\right\rangle d s+2 \int_{0}^{t}\left\langle\left(\Phi\left(u_{h^{\varepsilon}}^{\eta, R}\right)-\Phi\left(u_{h}^{\eta, R}\right)\right) h^{\varepsilon}(s), u_{h^{\varepsilon}}^{\eta, R}-u_{h}^{\eta, R}\right\rangle d s \\
& +2 \int_{0}^{t}\left\langle\Phi\left(u_{h}^{\eta, R}\right)\left(h^{\varepsilon}(s)-h(s)\right), u_{h^{\varepsilon}}^{\eta, R}-u_{h}^{\eta, R}\right\rangle d s \\
:= & I_{1}+I_{2}+2 \int_{0}^{t}\left\langle\Phi\left(u_{h}^{\eta, R}\right)\left(h^{\varepsilon}(s)-h(s)\right), u_{h^{\varepsilon}}^{\eta, R}-u_{h}^{\eta, R}\right\rangle d s .
\end{aligned}
$$


Using the Hölder inequality and Lipschitz continuous of $A^{R}$, we get

$$
\begin{aligned}
I_{1} & \leq 2 R \int_{0}^{t}\left\|\nabla\left(u_{h^{\varepsilon}}^{\eta, R}-u_{h}^{\eta, R}\right)\right\|_{H}\left\|u_{h^{\varepsilon}}^{\eta, R}-u_{h}^{\eta, R}\right\|_{H} d s \\
& \leq \eta \int_{0}^{t}\left\|\nabla\left(u_{h^{\varepsilon}}^{\eta, R}-u_{h}^{\eta, R}\right)\right\|_{H}^{2} d s+C(\eta, R) \int_{0}^{t}\left\|u_{h^{\varepsilon}}^{\eta, R}-u_{h}^{\eta, R}\right\|_{H}^{2} d s .
\end{aligned}
$$

Using Hölder inequality and (2.15), we obtain

$$
\begin{aligned}
I_{2} & \leq 2 \int_{0}^{t}\left\|\Phi\left(u_{h^{\varepsilon}}^{\eta, R}\right)-\Phi\left(u_{h}^{\eta, R}\right)\right\|_{\mathcal{L}_{2}(U, H)}\left|h^{\varepsilon}(s)\right|_{U}\left\|u_{h^{\varepsilon}}^{\eta, R}-u_{h}^{\eta, R}\right\|_{H} d s \\
& \leq \sqrt{D_{1}} \int_{0}^{t}\left\|u_{h^{\varepsilon}}^{\eta, R}-u_{h}^{\eta, R}\right\|_{H}^{2}\left|h^{\varepsilon}(s)\right|_{U} d s .
\end{aligned}
$$

Hence, it follows that

$$
\begin{aligned}
& \sup _{t \in[0, T]}\left\|u_{h^{\varepsilon}}^{\eta, R}(t)-u_{h}^{\eta, R}(t)\right\|_{H}^{2}+\eta \int_{0}^{T}\left\|\nabla\left(u_{h^{\varepsilon}}^{\eta, R}-u_{h}^{\eta, R}\right)\right\|_{H}^{2} d s \\
& \leq \quad C(\eta, R) \int_{0}^{T}\left\|u_{h^{\varepsilon}}^{\eta, R}-u_{h}^{\eta, R}\right\|_{H}^{2}\left(1+\left|h^{\varepsilon}(s)\right|_{U}\right) d s \\
& +2 \sup _{t \in[0, T]}\left|\int_{0}^{t}\left\langle\Phi\left(u_{h}^{\eta, R}\right)\left(h^{\varepsilon}(s)-h(s)\right), u_{h^{\varepsilon}}^{\eta, R}-u_{h}^{\eta, R}\right\rangle d s\right| .
\end{aligned}
$$

By the Gronwall inequality, we obtain

$$
\begin{aligned}
& \sup _{t \in[0, T]}\left\|u_{h^{\varepsilon}}^{\eta, R}(t)-u_{h}^{\eta, R}(t)\right\|_{H}^{2}+\eta \int_{0}^{T}\left\|\nabla\left(u_{h^{\varepsilon}}^{\eta, R}-u_{h}^{\eta, R}\right)\right\|_{H}^{2} d s \\
\leq & 2 \sup _{t \in[0, T]}\left|\int_{0}^{t}\left\langle\Phi\left(u_{h}^{\eta, R}\right)\left(h^{\varepsilon}(s)-h(s)\right), u_{h^{\varepsilon}}^{\eta, R}-u_{h}^{\eta, R}\right\rangle d s\right| \exp \left\{C(\eta, R) \int_{0}^{T}\left(1+\left|h^{\varepsilon}(s)\right|_{U}^{2}\right) d s\right\} \\
\leq & C(\eta, R, T, M) \sup _{t \in[0, T]}\left|\int_{0}^{t}\left\langle\Phi\left(u_{h}^{\eta, R}\right)\left(h^{\varepsilon}(s)-h(s)\right), u_{h^{\varepsilon}}^{\eta, R}-u_{h}^{\eta, R}\right\rangle d s\right| .
\end{aligned}
$$

To show $\lim _{\varepsilon \rightarrow 0} \sup _{t \in[0, T]}\left\|u_{h^{\varepsilon}}^{\eta, R}(t)-u_{h}^{\eta, R}(t)\right\|_{H}^{2}=0$, it suffices to prove that

$$
\lim _{\varepsilon \rightarrow 0} \sup _{0 \leq t \leq T}\left|\int_{0}^{t}\left\langle\Phi\left(u_{h}^{\eta, R}\right)\left(h^{\varepsilon}-h\right), u_{h^{\varepsilon}}^{\eta, R}-u_{h}^{\eta, R}\right\rangle d s\right|=0 .
$$

This will be achieved if we show that for any sequence $\varepsilon_{m} \rightarrow 0$, one can find a subsequence $\varepsilon_{m_{k}} \rightarrow 0$ such that

$$
\lim _{k \rightarrow \infty} \sup _{0 \leq t \leq T}\left|\int_{0}^{t}\left\langle\Phi\left(u_{h}^{\eta, R}\right)\left(h^{\varepsilon_{m_{k}}}-h\right), u_{h^{\varepsilon_{m_{k}}}}^{\eta, R}-u_{h}^{\eta, R}\right\rangle d s\right|=0
$$

Now fix a sequence $\varepsilon_{m} \rightarrow 0$. Since $\left\{u_{h^{\varepsilon m}}^{\eta, R}, m \geq 1\right\}$ is compact in $L^{2}([0, T] ; H)$, there exists a subsequence 
$\left\{m_{k}, k \geq 1\right\}$ and a mapping $\tilde{u}$ such that $u_{h^{\varepsilon m_{k}}}^{\eta, R} \rightarrow \tilde{u}$ in $L^{2}([0, T] ; H)$. Now, note that

$$
\begin{aligned}
& \sup _{0 \leq t \leq T}\left|\int_{0}^{t}\left\langle\Phi\left(u_{h}^{\eta, R}\right)\left(h^{\varepsilon_{m_{k}}}-h\right), u_{h^{\varepsilon_{m_{k}}}}^{\eta, R}-u_{h}^{\eta, R}\right\rangle d s\right| \\
\leq & \sup _{0 \leq t \leq T}\left|\int_{0}^{t}\left\langle\Phi\left(u_{h}^{\eta, R}\right)\left(h^{\varepsilon_{m_{k}}}-h\right), u_{h^{\varepsilon_{m_{k}}}}^{\eta, R}-\tilde{u}\right\rangle d s\right| \\
& +\sup _{0 \leq t \leq T}\left|\int_{0}^{t}\left\langle\Phi\left(u_{h}^{\eta, R}\right)\left(h^{\varepsilon_{m_{k}}}-h\right), \tilde{u}-u_{h}^{\eta, R}\right\rangle d s\right| .
\end{aligned}
$$

Since $h^{\varepsilon_{m_{k}}} \rightarrow h$ weakly in $L^{2}([0, T] ; U)$, for every $t>0$, it follows that

$$
\int_{0}^{t}\left\langle\Phi\left(u_{h}^{\eta, R}\right)\left(h^{\varepsilon_{m_{k}}}-h\right), \tilde{u}-u_{h}^{\eta, R}\right\rangle d s=0 .
$$

On the other hand, by (2.14) and utilizing the assumption on $h$, for $0<t_{1}<t_{2} \leq T$, it yields

$$
\begin{aligned}
& \left|\int_{t_{1}}^{t_{2}}\left\langle\Phi\left(u_{h}^{\eta, R}\right)\left(h^{\varepsilon_{m_{k}}}-h\right), \tilde{u}-u_{h}^{\eta, R}\right\rangle d s\right| \\
\leq & \int_{t_{1}}^{t_{2}}\left\|\tilde{u}-u_{h}^{\eta, R}\right\|_{H}\left\|\Phi\left(u_{h}^{\eta, R}\right)\right\|_{\mathcal{L}_{2}(U, H)}\left|h^{\varepsilon_{m_{k}}}-h\right|_{U} d s \\
\leq & \sqrt{D_{0}} \int_{t_{1}}^{t_{2}}\left\|\tilde{u}-u_{h}^{\eta, R}\right\|_{H}\left(1+\left\|u_{h}^{\eta, R}\right\|_{H}\right)\left|h^{\varepsilon_{m_{k}}}-h\right|_{U} d s \\
\leq & \sqrt{D_{0}}(2 M)^{\frac{1}{2}}\left(1+\sup _{t \in[0, T]}\left\|u_{h}^{\eta, R}\right\|_{H}\right)\left(\int_{t_{1}}^{t_{2}}\left\|\tilde{u}-u_{h}^{\eta, R}\right\|_{H}^{2} d s\right)^{\frac{1}{2}} \\
\leq & \sqrt{D_{0}} C\left(M, T,\left\|u_{0}\right\|_{H}\right)\left(\int_{t_{1}}^{t_{2}}\left\|\tilde{u}-u_{h}^{\eta, R}\right\|_{H}^{2} d s\right)^{\frac{1}{2}} .
\end{aligned}
$$

Combing (4.58) and (4.59), we deduce that

$$
\lim _{k \rightarrow \infty} \sup _{0 \leq t \leq T}\left|\int_{0}^{t}\left\langle\Phi\left(u_{h}^{\eta, R}\right)\left(h^{\varepsilon_{m_{k}}}-h\right), \tilde{u}-u_{h}^{\eta, R}\right\rangle d s\right|=0 .
$$

By Hölder inequality, we have

$$
\sup _{t \in[0, T]}\left|\int_{0}^{t}\left\langle\Phi\left(u_{h}^{\eta, R}\right)\left(h^{\varepsilon_{m_{k}}}-h\right), u_{h^{\varepsilon_{m_{k}}}}^{\eta, R}-\tilde{u}\right\rangle d s\right| \leq \sqrt{D_{0}} C\left(M, T,\left\|u_{0}\right\|_{H}\right)\left(\int_{0}^{T}\left\|u_{h^{\varepsilon m_{k}}}^{\eta, R}-\tilde{u}\right\|_{H}^{2} d s\right)^{\frac{1}{2}} .
$$

Since $u_{h^{\varepsilon_{m_{k}}}}^{\eta, R} \rightarrow \tilde{u}$ in $L^{2}([0, T] ; H)$, we obtain

$$
\lim _{k \rightarrow \infty} \sup _{t \in[0, T]}\left|\int_{0}^{t}\left\langle\Phi\left(u_{h}^{\eta, R}\right)\left(h^{\varepsilon_{m_{k}}}-h\right), u_{h^{\varepsilon_{m_{k}}}}^{\eta, R}-\tilde{u}\right\rangle d s\right|=0 .
$$

Collecting the above estimates, we prove (4.57). Hence

$$
\lim _{\varepsilon \rightarrow 0} \sup _{t \in[0, T]}\left\|u_{h^{\varepsilon}}^{\eta, R}(t)-u_{h}^{\eta, R}(t)\right\|_{H}^{2}=0
$$


which further implies that for any $\eta>0, R>0$,

$$
\lim _{\varepsilon \rightarrow 0}\left\|u_{h^{\varepsilon}}^{\eta, R}-u_{h}^{\eta, R}\right\|_{L^{1}\left([0, T] ; L^{1}\left(\mathbb{T}^{N}\right)\right)}=0 .
$$

Note that for any $\varepsilon, \eta, R>0$,

$$
\begin{aligned}
& \left\|u_{h^{\varepsilon}}-u_{h}\right\|_{L^{1}\left([0, T] ; L^{1}\left(\mathbb{T}^{N}\right)\right)} \\
\leq \quad & \left\|u_{h^{\varepsilon}}^{\eta}-u_{h^{\varepsilon}}\right\|_{L^{1}\left([0, T] ; L^{1}\left(\mathbb{T}^{N}\right)\right)}+\left\|u_{h^{\varepsilon}}^{\eta}-u_{h^{\varepsilon}}^{\eta, R}\right\|_{L^{1}\left([0, T] ; L^{1}\left(\mathbb{T}^{N}\right)\right)}+\left\|u_{h^{\varepsilon}}^{\eta, R}-u_{h}^{\eta, R}\right\|_{L^{1}\left([0, T] ; L^{1}\left(\mathbb{T}^{N}\right)\right)} \\
& +\left\|u_{h}^{\eta, R}-u_{h}^{\eta}\right\|_{L^{1}\left([0, T] ; L^{1}\left(\mathbb{T}^{N}\right)\right)}+\left\|u_{h}^{\eta}-u_{h}\right\|_{L^{1}\left([0, T] ; L^{1}\left(\mathbb{T}^{N}\right)\right)} .
\end{aligned}
$$

For any $\iota>0$, by Proposition 4.5 , there exists $\eta_{0}$ such that for all $\varepsilon>0$,

$$
\left\|u_{h^{\varepsilon}}^{\eta_{0}}-u_{h^{\varepsilon}}\right\|_{L^{1}\left([0, T] ; L^{1}\left(\mathbb{T}^{N}\right)\right)} \leq \frac{\iota}{4} \text { and }\left\|u_{h}^{\eta_{0}}-u_{h}\right\|_{L^{1}\left([0, T] ; L^{1}\left(\mathbb{T}^{N}\right)\right)} \leq \frac{\iota}{4}
$$

Letting $\eta=\eta_{0}$, we deduce from (4.61) that

$$
\begin{aligned}
\left\|u_{h^{\varepsilon}}-u_{h}\right\|_{L^{1}\left([0, T] ; L^{1}\left(\mathbb{T}^{N}\right)\right)} \leq & \frac{\iota}{2}+\left\|u_{h^{\varepsilon}}^{\eta_{0}}-u_{h^{\varepsilon}}^{\eta_{0}, R}\right\|_{L^{1}\left([0, T] ; L^{1}\left(\mathbb{T}^{N}\right)\right)}+\left\|u_{h^{\varepsilon}}^{\eta_{0}, R}-u_{h}^{\eta_{0}, R}\right\|_{L^{1}\left([0, T] ; L^{1}\left(\mathbb{T}^{N}\right)\right)} \\
& +\left\|u_{h}^{\eta_{0}, R}-u_{h}^{\eta_{0}}\right\|_{L^{1}\left([0, T] ; L^{1}\left(\mathbb{T}^{N}\right)\right)} .
\end{aligned}
$$

Using (4.46), there exists $R_{0}$ large enough such that for all $\varepsilon>0$,

$$
\left\|u_{h^{\varepsilon}}^{\eta_{0}}-u_{h^{\varepsilon}}^{\eta_{0}, R_{0}}\right\|_{L^{1}\left([0, T] ; L^{1}\left(\mathbb{T}^{N}\right)\right)} \leq \frac{\iota}{4} \text { and }\left\|u_{h}^{\eta_{0}, R_{0}}-u_{h}^{\eta_{0}}\right\|_{L^{1}\left([0, T] ; L^{1}\left(\mathbb{T}^{N}\right)\right)} \leq \frac{\iota}{4} .
$$

Replacing $R$ by $R_{0}$ in (4.62), we get

$$
\left\|u_{h^{\varepsilon}}-u_{h}\right\|_{L^{1}\left([0, T] ; L^{1}\left(\mathbb{T}^{N}\right)\right)} \leq \iota+\left\|u_{h^{\varepsilon}}^{\eta_{0}, R_{0}}-u_{h}^{\eta_{0}, R_{0}}\right\|_{L^{1}\left([0, T] ; L^{1}\left(\mathbb{T}^{N}\right)\right)} .
$$

Using (4.60), we conclude that

$$
\lim _{\varepsilon \rightarrow 0}\left\|u_{h^{\varepsilon}}-u_{h}\right\|_{L^{1}\left([0, T] ; L^{1}\left(\mathbb{T}^{N}\right)\right)} \leq \iota .
$$

Since the constant $\iota$ is arbitrary, we obtain the desired result.

\section{Large deviations}

For any family $\left\{h^{\varepsilon} ; 0<\varepsilon<1\right\} \subset \mathcal{A}_{M}$ with $h^{\varepsilon}=\sum_{k \geq 1} h^{\varepsilon, k} e_{k}$, we consider the following equation

$$
\left\{\begin{array}{l}
d \bar{u}^{\varepsilon}+\operatorname{div}\left(A\left(\bar{u}^{\varepsilon}\right)\right) d t=\Phi\left(\bar{u}^{\varepsilon}\right) h^{\varepsilon}(t) d t+\sqrt{\varepsilon} \Phi\left(\bar{u}^{\varepsilon}\right) d W(t), \\
\bar{u}^{\varepsilon}(0)=u_{0} .
\end{array}\right.
$$

Combing Theorem 2.5 and Theorem 4.3, we conclude that there exists a unique kinetic solution $\bar{u}^{\varepsilon}$ with initial data $u_{0} \in L^{\infty}\left(\mathbb{T}^{N}\right)$ satisfying the following

$$
\mathbb{E}\left(\text { ess } \sup _{t \in[0, T]}\left\|\bar{u}^{\varepsilon}(t)\right\|_{L^{1}\left(\mathbb{T}^{N}\right)}\right)<+\infty
$$


and there exists a kinetic measure $\bar{m}^{\varepsilon} \in \mathcal{M}_{0}^{+}\left(\mathbb{T}^{N} \times[0, T] \times \mathbb{R}\right)$ such that $\bar{f}^{\varepsilon}:=I_{\bar{u}^{\varepsilon}>\xi}$ fulfills that for all $\varphi \in C_{c}^{1}\left(\mathbb{T}^{N} \times[0, T) \times \mathbb{R}\right)$,

$$
\begin{aligned}
& \int_{0}^{T}\left\langle\bar{f}^{\varepsilon}(t), \partial_{t} \varphi(t)\right\rangle d t+\left\langle f_{0}, \varphi(0)\right\rangle+\int_{0}^{T}\left\langle\bar{f}^{\varepsilon}(t), a(\xi) \cdot \nabla \varphi(t)\right\rangle d t \\
= & -\sqrt{\varepsilon} \sum_{k \geq 1} \int_{0}^{T} \int_{\mathbb{T}^{N}} \int_{\mathbb{R}} g_{k}\left(x, \bar{u}^{\varepsilon}(x, t)\right) \varphi\left(x, t, \bar{u}^{\varepsilon}(x, t)\right) d x d \beta_{k}(t) \\
& -\frac{\varepsilon}{2} \int_{0}^{T} \int_{\mathbb{T}^{N}} \partial_{\xi} \varphi\left(x, t, \bar{u}^{\varepsilon}(x, t)\right) G^{2}\left(x, \bar{u}^{\varepsilon}(x, t)\right) d x d t \\
& -\sum_{k \geq 1} \int_{0}^{T} \int_{\mathbb{T}^{N}} \int_{\mathbb{R}} \varphi\left(x, t, \bar{u}^{\varepsilon}(x, t)\right) g_{k}\left(x, \bar{u}^{\varepsilon}(x, t)\right) h^{\varepsilon, k}(t) d x d t+\bar{m}^{\varepsilon}\left(\partial_{\xi} \varphi\right), \text { a.s. }
\end{aligned}
$$

where $G^{2}:=\sum_{k \geq 1}\left|g_{k}\right|^{2}$. According to the definition of $\mathcal{G}^{\varepsilon}$, it is clear that $\mathcal{G}^{\varepsilon}\left(W(\cdot)+\frac{1}{\sqrt{\varepsilon}} \int_{0}^{\cdot} h^{\varepsilon}(s) d s\right)=\bar{u}^{\varepsilon}(\cdot)$.

According to Theorem 3.1 (the sufficient condition B) and Theorem 4.6, we only need to prove the following result to establish the main result.

Theorem 5.1. For every $M<\infty$, let $\left\{h^{\varepsilon}: \varepsilon>0\right\} \subset \mathcal{A}_{M}$. Then

$$
\left\|\mathcal{G}^{\varepsilon}\left(W(\cdot)+\frac{1}{\sqrt{\varepsilon}} \int_{0}^{\cdot} h^{\varepsilon}(s) d s\right)-\mathcal{G}^{0}\left(\int_{0} h^{\varepsilon}(s) d s\right)\right\|_{L^{1}\left([0, T] ; L^{1}\left(\mathbb{T}^{N}\right)\right)} \rightarrow 0 \quad \text { in probability. }
$$

Proof. Recall that $\bar{u}^{\varepsilon}=\mathcal{G}^{\varepsilon}\left(W(\cdot)+\frac{1}{\sqrt{\varepsilon}} \int_{0}^{\infty} h^{\varepsilon}(s) d s\right)$ is the kinetic solution to (5.63) with the corresponding kinetic measure $m_{1}^{\varepsilon}$. Moreover, $v^{\varepsilon}:=\mathcal{G}^{0}\left(\int_{0}^{*} h^{\varepsilon}(s) d s\right)$ is the kinetic solution to the skeleton equation (3.18) with $h$ replaced by $h^{\varepsilon}$ and the corresponding kinetic measure is denoted by $\bar{m}_{2}^{\varepsilon}$.

Denote $f_{1}(x, t, \xi):=I_{\bar{u}^{\varepsilon}(x, t)>\xi}$ and $f_{2}(y, t, \zeta):=I_{v^{\varepsilon}(y, t)>\zeta}$. Using the same procedure as for (4.22)-(4.23), we have for all $\varphi_{1}(x, \xi) \in C_{c}^{\infty}\left(\mathbb{T}_{x}^{N} \times \mathbb{R}_{\xi}\right)$,

$$
\begin{aligned}
\left\langle f_{1}^{ \pm}(t), \varphi_{1}\right\rangle= & \left\langle f_{1,0}, \varphi_{1}\right\rangle+\int_{0}^{t}\left\langle f_{1}(s), a(\xi) \cdot \nabla_{x} \varphi_{1}(x, \xi)\right\rangle d s \\
& +\sqrt{\varepsilon} \sum_{k \geq 1} \int_{0}^{t} \int_{\mathbb{T}^{N}} \int_{\mathbb{R}} g_{k}(x, \xi) \varphi_{1}(x, \xi) d v_{x, s}^{1, \varepsilon}(\xi) d x d \beta_{k}(s) \\
& +\frac{\varepsilon}{2} \int_{0}^{t} \int_{\mathbb{T}^{N}} \int_{\mathbb{R}} \partial_{\xi} \varphi_{1}(x, \xi) G^{2}(x, \xi) d v_{x, s}^{1, \varepsilon}(\xi) d x d s \\
& +\sum_{k \geq 1} \int_{0}^{t} \int_{\mathbb{T}^{N}} \int_{\mathbb{R}} \varphi_{1}(x, \xi) g_{k}(x, \xi) h^{\varepsilon, k}(s) d v_{x, s}^{1, \varepsilon}(\xi) d x d s-\left\langle m_{1}^{\varepsilon}, \partial_{\xi} \varphi_{1}\right\rangle([0, t]),
\end{aligned}
$$

where $f_{1,0}=I_{u_{0}>\xi}$ and $v_{x, s}^{1, \varepsilon}(\xi)=-\partial_{\xi} f_{1}^{ \pm}(s, x, \xi)=\partial_{\xi} \bar{f}_{1}^{ \pm}(s, x, \xi)=\delta_{\bar{u}^{\varepsilon, \pm}(x, t)=\xi}$. Similarly, in view of (5.64), for all $\varphi_{2}(y, \zeta) \in C_{c}^{\infty}\left(\mathbb{T}_{y}^{N} \times \mathbb{R}_{\zeta}\right)$, we have

$$
\begin{aligned}
\left\langle\bar{f}_{2}^{ \pm}(t), \varphi_{2}\right\rangle= & \left\langle\bar{f}_{2,0}, \varphi_{2}\right\rangle+\int_{0}^{t}\left\langle\bar{f}_{2}(s), a(\zeta) \cdot \nabla_{y} \varphi_{2}(y, \zeta)\right\rangle d s \\
& -\sum_{k \geq 1} \int_{0}^{t} \int_{\mathbb{T}^{N}} \int_{\mathbb{R}} g_{k}(y, \zeta) \varphi_{2}(y, \zeta) h^{\varepsilon, k}(s) d \bar{v}_{y, s}^{2, \varepsilon}(\zeta) d y d s+\left\langle\bar{m}_{2}^{\varepsilon}, \partial_{\zeta} \varphi_{2}\right\rangle([0, t]),
\end{aligned}
$$


where $f_{2,0}=I_{u_{0}>\zeta}$ and $\bar{v}_{y, s}^{2, \varepsilon}(\zeta)=\partial_{\zeta} \bar{f}_{2}^{ \pm}(s, y, \zeta)=-\partial_{\zeta} f_{2}^{ \pm}(s, y, \zeta)=\delta_{v^{\varepsilon, \pm}}(y, t)=\zeta$.

Setting $\alpha(x, \xi, y, \zeta)=\varphi_{1}(x, \xi) \varphi_{2}(y, \zeta)$, using integration by parts formula, we deduce that

$$
\begin{aligned}
& \left\langle\left\langle f_{1}^{ \pm}(t) \bar{f}_{2}^{ \pm}(t), \alpha\right\rangle\right\rangle \\
= & \left\langle\left\langle f_{1,0} \bar{f}_{2,0}, \alpha\right\rangle\right\rangle+\int_{0}^{t} \int_{\left(\mathbb{T}^{N}\right)^{2}} \int_{\mathbb{R}^{2}} f_{1} \bar{f}_{2}(a(\xi)-a(\zeta)) \cdot \nabla_{x} \alpha d \xi d \zeta d x d y d s \\
& +\frac{\varepsilon}{2} \int_{0}^{t} \int_{\left(\mathbb{T}^{N}\right)^{2}} \int_{\mathbb{R}^{2}} \partial_{\xi} \alpha \bar{f}_{2}^{ \pm}(s, y, \zeta) G^{2}(x, \xi) d v_{x, s}^{1, \varepsilon}(\xi) d \zeta d x d y d s \\
& +\sum_{k \geq 1} \int_{0}^{t} \int_{\left(\mathbb{T}^{N}\right)^{2}} \int_{\mathbb{R}^{2}} \bar{f}_{2}^{ \pm}(s, y, \zeta) \alpha g_{k}(x, \xi) h^{\varepsilon, k}(s) d \zeta d v_{x, s}^{1, \varepsilon}(\xi) d x d y d s \\
& -\sum_{k \geq 1} \int_{0}^{t} \int_{\left(\mathbb{T}^{N}\right)^{2}} \int_{\mathbb{R}^{2}} f_{1}^{ \pm}(s, x, \xi) \alpha g_{k}(y, \zeta) h^{\varepsilon, k}(s) d \xi d \bar{v}_{y, s}^{2, \varepsilon}(\zeta) d x d y d s \\
& -\int_{0}^{t} \int_{\left(\mathbb{T}^{N}\right)^{2}} \int_{\mathbb{R}^{2}} \bar{f}_{2}^{ \pm}(s, y, \zeta) \partial_{\xi} \alpha d m_{1}^{\varepsilon}(x, \xi, s) d \zeta d y \\
& +\int_{0}^{t} \int_{\left(\mathbb{T}^{N}\right)^{2}} \int_{\mathbb{R}^{2}} f_{1}^{ \pm}(s, x, \xi) \partial_{\zeta} \alpha d \bar{m}_{2}^{\varepsilon}(y, \zeta, s) d \xi d x \\
& +\sqrt{\varepsilon} \sum_{k \geq 1} \int_{0}^{t} \int_{\left(\mathbb{T}^{N}\right)^{2}} \int_{\mathbb{R}^{2}} \bar{f}_{2}^{ \pm}(s, y, \zeta) g_{k}(x, \xi) \alpha d \zeta d v_{x, s}^{1, \varepsilon}(\xi) d x d y d \beta_{k}(s) \\
:= & \left\langle\left\langle f_{1,0} \bar{f}_{2,0}, \alpha\right\rangle\right\rangle+J_{1}+J_{2}+J_{3}+J_{4}+J_{5}+J_{6}+J_{7} .
\end{aligned}
$$

Similarly, we get

$$
\begin{aligned}
& \left\langle\left\langle\bar{f}_{1}^{ \pm}(t) f_{2}^{ \pm}(t), \alpha\right\rangle\right\rangle \\
= & \left\langle\left\langle\bar{f}_{1,0} f_{2,0}, \alpha\right\rangle\right\rangle+\int_{0}^{t} \int_{\left(\mathbb{T}^{N}\right)^{2}} \int_{\mathbb{R}^{2}} \bar{f}_{1} f_{2}(a(\xi)-a(\zeta)) \cdot \nabla_{x} \alpha d \xi d \zeta d x d y d s \\
& -\frac{\varepsilon}{2} \int_{0}^{t} \int_{\left(\mathbb{T}^{N}\right)^{2}} \int_{\mathbb{R}^{2}} \partial_{\xi} \alpha f_{2}^{ \pm}(s, y, \zeta) G^{2}(x, \xi) d v_{x, s}^{1, \varepsilon}(\xi) d \zeta d x d y d s \\
& -\sum_{k \geq 1} \int_{0}^{t} \int_{\left(\mathbb{T}^{N}\right)^{2}} \int_{\mathbb{R}^{2}} f_{2}^{ \pm}(s, y, \zeta) \alpha g_{k}(x, \xi) h^{\varepsilon, k}(s) d \zeta d v_{x, s}^{1, \varepsilon}(\xi) d x d y d s \\
& +\sum_{k \geq 1} \int_{0}^{t} \int_{\left(\mathbb{T}^{N}\right)^{2}} \int_{\mathbb{R}^{2}} \bar{f}_{1}^{ \pm}(s, x, \xi) \alpha g_{k}(y, \zeta) h^{\varepsilon, k}(s) d \xi d \bar{v}_{y, s}^{2, \varepsilon}(\zeta) d x d y d s \\
& +\int_{0}^{t} \int_{\left(\mathbb{T}^{N}\right)^{2}} \int_{\mathbb{R}^{2}} f_{2}^{ \pm}(s, y, \zeta) \partial_{\xi} \alpha d m_{1}^{\varepsilon}(x, \xi, s) d \zeta d y \\
& -\int_{0}^{t} \int_{\left(\mathbb{T}^{N}\right)^{2}} \int_{\mathbb{R}^{2}} \bar{f}_{1}^{ \pm}(s, x, \xi) \partial_{\zeta} \alpha d \bar{m}_{2}^{\varepsilon}(y, \zeta, s) d \xi d x \\
& -\sqrt{\varepsilon}_{\varepsilon} \int_{k \geq 1}^{t} \int_{\left(\mathbb{T}^{N}\right)^{2}} \int_{\mathbb{R}^{2}} f_{2}^{ \pm}(s, y, \zeta) g_{k}(x, \xi) \alpha d \zeta d v_{x, s}^{1, \varepsilon}(\xi) d x d y d \beta_{k}(s) \\
:= & \left\langle\left\langle\bar{f}_{1,0} f_{2,0}, \alpha\right\rangle\right\rangle+\bar{J}_{1}+\bar{J}_{2}+\bar{J}_{3}+\bar{J}_{4}+\bar{J}_{5}+\bar{J}_{6}+\bar{J}_{7} .
\end{aligned}
$$

Taking $\alpha(x, y, \xi, \zeta)=\rho_{\gamma}(x-y) \psi_{\delta}(\xi-\zeta)$, where $\rho_{\gamma}$ and $\psi_{\delta}$ are approximations to the identity on $\mathbb{T}^{N}$ 
and $\mathbb{R}$, respectively. Then, we have

$$
\begin{aligned}
& \int_{\left(\mathbb{T}^{N}\right)^{2}} \int_{\mathbb{R}^{2}} \rho_{\gamma}(x-y) \psi_{\delta}(\xi-\zeta)\left(f_{1}^{ \pm}(x, t, \xi) \bar{f}_{2}^{ \pm}(y, t, \zeta)+\bar{f}_{1}^{ \pm}(x, t, \xi) f_{2}^{ \pm}(y, t, \zeta)\right) d \xi d \zeta d x d y \\
\leq & \int_{\left(\mathbb{T}^{N}\right)^{2}} \int_{\mathbb{R}^{2}} \rho_{\gamma}(x-y) \psi_{\delta}(\xi-\zeta)\left(f_{1,0}(x, \xi) \bar{f}_{2,0}(y, \zeta)+\bar{f}_{1,0}(x, \xi) f_{2,0}(y, \zeta)\right) d \xi d \zeta d x d y \\
& +\sum_{i=1}^{7}\left(\tilde{J}_{i}+\tilde{\tilde{J}}_{i}\right),
\end{aligned}
$$

where $\tilde{J}_{i}, \tilde{\bar{J}}_{i}$ in (5.65) are the corresponding $J_{i}, \bar{J}_{i}$ with $\alpha(x, y, \xi, \zeta)=\rho_{\gamma}(x-y) \psi_{\delta}(\xi-\zeta)$, for $i=1, \cdots, 7$.

By the same method as the proof of Theorem 15 in [9], we have

$$
\left|\tilde{J}_{1}\right| \leq T C_{p} \delta \gamma^{-1}, \quad \tilde{J}_{5}+\tilde{J}_{6} \leq 0, \quad\left|\tilde{\bar{J}}_{1}\right| \leq T C_{p} \delta \gamma^{-1}, \quad \tilde{\bar{J}}_{5}+\tilde{\bar{J}}_{6} \leq 0 .
$$

With the aid of $\gamma_{1}(\xi, \zeta), \gamma_{2}(\xi, \zeta)$ and by using (2.12), we have

$$
\begin{aligned}
\tilde{\bar{J}}_{2}= & \tilde{J}_{2} \\
= & \frac{\varepsilon}{2} \int_{0}^{t} \int_{\left(\mathbb{T}^{N}\right)^{2}} \int_{\mathbb{R}^{2}} \alpha G^{2}(x, \xi) d v_{x, s}^{1, \varepsilon} \otimes d \bar{v}_{y, s}^{2, \varepsilon}(\xi, \zeta) d x d y d s \\
\leq & \frac{\varepsilon}{2} D_{0} \int_{0}^{t} \int_{\left(\mathbb{T}^{N}\right)^{2}} \int_{\mathbb{R}^{2}} \alpha\left(1+|\xi|^{2}\right) d v_{x, s}^{1, \varepsilon} \otimes d \bar{v}_{y, s}^{2, \varepsilon}(\xi, \zeta) d x d y d s \\
\leq & \frac{\varepsilon}{2} D_{0} \int_{0}^{t} \int_{\left(\mathbb{T}^{N}\right)^{2}} \int_{\mathbb{R}^{2}} \alpha d v_{x, s}^{1, \varepsilon} \otimes d \bar{v}_{y, s}^{2, \varepsilon}(\xi, \zeta) d x d y d s \\
& +\frac{\varepsilon}{2} D_{0} \int_{0}^{t} \int_{\left(\mathbb{T}^{N}\right)^{2}} \int_{\mathbb{R}^{2}} \alpha|\xi|^{2} d v_{x, s}^{1, \varepsilon} \otimes d \bar{v}_{y, s}^{2, \varepsilon}(\xi, \zeta) d x d y d s .
\end{aligned}
$$

Clearly, it holds that

$$
\begin{aligned}
& \int_{\left(\mathbb{T}^{N}\right)^{2}} \int_{\mathbb{R}^{2}} \alpha d v_{x, s}^{1, \varepsilon} \otimes d \bar{v}_{y, s}^{2, \varepsilon}(\xi, \zeta) d x d y \\
\leq & \left\|\psi_{\delta}\right\|_{L^{\infty}} \int_{\left.\mathbb{T}^{N}\right)^{2}} \int_{\mathbb{R}^{2}} \rho_{\gamma}(x-y) d v_{x, s}^{1, \varepsilon} \otimes d \bar{v}_{y, s}^{2, \varepsilon}(\xi, \zeta) d x d y \\
\leq & \left\|\psi_{\delta}\right\|_{L^{\infty}} \int_{\left(\mathbb{T}^{N}\right)^{2}} \rho_{\gamma}(x-y) d x d y \\
\leq & \delta^{-1} .
\end{aligned}
$$

Moreover, by utilizing the property that measures $v_{x, s}^{1, \varepsilon}$ and $\bar{v}_{y, s}^{2, \varepsilon}$ vanish at infinity, it yields that

$$
\begin{aligned}
& \int_{\left(\mathbb{T}^{N}\right)^{2}} \int_{\mathbb{R}^{2}} \alpha|\xi|^{2} d v_{x, s}^{1, \varepsilon} \otimes d \bar{v}_{y, s}^{2, \varepsilon}(\xi, \zeta) d x d y \\
\leq & \int_{\left(\mathbb{T}^{N}\right)^{2}} \rho_{\gamma}(x-y) \int_{\mathbb{R}^{2}} \psi_{\delta}(\xi-\zeta)|\xi|^{2} d v_{x, s}^{1, \varepsilon} \otimes d \bar{v}_{y, s}^{2, \varepsilon}(\xi, \zeta) d x d y \\
\leq & \left\|\psi_{\delta}\right\|_{L^{\infty}} \int_{\left(\mathbb{T}^{N}\right)^{2}} \rho_{\gamma}(x-y) \int_{\mathbb{R}^{2}}|\xi|^{2} d v_{x, s}^{1, \varepsilon} \otimes d \bar{v}_{y, s}^{2, \varepsilon}(\xi, \zeta) d x d y \\
\leq & C \delta^{-1} \int_{\left(\mathbb{T}^{N}\right)^{2}} \rho_{\gamma}(x-y) d x d y \\
\leq & C \delta^{-1} .
\end{aligned}
$$


Hence, combing (5.66) and (5.67), we deduce that

$$
\tilde{\bar{J}_{2}}=\tilde{J}_{2} \leq \frac{\varepsilon}{2} D_{0} T \delta^{-1}+\frac{\varepsilon}{2} C D_{0} T \delta^{-1} \leq \varepsilon C D_{0} T \delta^{-1} .
$$

Recall

$$
\gamma_{2}(\zeta, \xi)=\int_{\zeta}^{\infty} \psi_{\delta}\left(\xi-\zeta^{\prime}\right) d \zeta^{\prime}
$$

Using the similar arguments as in the proof of Proposition 4.2, we have

$$
\begin{aligned}
\tilde{\bar{J}}_{3}+\tilde{\bar{J}}_{4}= & \tilde{J}_{3}+\tilde{J}_{4} \\
= & \sum_{k \geq 1} \int_{0}^{t} \int_{\left(\mathbb{T}^{N}\right)^{2}} \int_{\mathbb{R}^{2}} \gamma_{2}(\zeta, \xi) \rho_{\gamma}(x-y)\left(g_{k}(x, \xi)-g_{k}(y, \zeta)\right) h^{\varepsilon, k}(s) d v_{x, s}^{1, \varepsilon} \otimes d \bar{v}_{y, s}^{2, \varepsilon}(\xi, \zeta) d x d y d s \\
\leq & \sum_{k \geq 1} \int_{0}^{t} \int_{\left(\mathbb{T}^{N}\right)^{2}} \int_{\mathbb{R}^{2}} \gamma_{2}(\zeta, \xi) \rho_{\gamma}(x-y)\left|g_{k}(x, \xi)-g_{k}(y, \zeta)\right|\left|h^{\varepsilon, k}(s)\right| d v_{x, s}^{1, \varepsilon} \otimes d \bar{v}_{y, s}^{2, \varepsilon}(\xi, \zeta) d x d y d s \\
\leq & \int_{0}^{t} \int_{\left(\mathbb{T}^{N}\right)^{2}} \int_{\mathbb{R}^{2}} \gamma_{2}(\zeta, \xi) \rho_{\gamma}(x-y)\left(\sum_{k \geq 1}\left|g_{k}(x, \xi)-g_{k}(y, \zeta)\right|^{2}\right)^{\frac{1}{2}}\left(\sum_{k \geq 1}\left|h^{\varepsilon, k}(s)\right|^{2}\right)^{\frac{1}{2}} d v_{x, s}^{1, \varepsilon} \otimes d \bar{v}_{y, s}^{2, \varepsilon}(\xi, \zeta) d x d y d s \\
\leq & \sqrt{D_{1}} \int_{0}^{t}\left|h^{\varepsilon}(s)\right|_{U} \int_{\left(\mathbb{T}^{N}\right)^{2}} \int_{\mathbb{R}^{2}} \gamma_{2}(\zeta, \xi) \rho_{\gamma}(x-y)|x-y| d v_{x, s}^{1, \varepsilon} \otimes d \bar{v}_{y, s}^{2, \varepsilon}(\xi, \zeta) d x d y d s \\
& +\sqrt{D_{1}} \int_{0}^{t}\left|h^{\varepsilon}(s)\right|_{U} \int_{\left(\mathbb{T}^{N}\right)^{2}} \rho_{\gamma}(x-y) \int_{\mathbb{R}^{2}} \gamma_{2}(\zeta, \xi)|\xi-\zeta| d v_{x, s}^{1, \varepsilon} \otimes d \bar{v}_{y, s}^{2, \varepsilon}(\xi, \zeta) d x d y d s \\
:= & \tilde{J}_{3,1}+\tilde{J}_{4,1} .
\end{aligned}
$$

By

$$
\begin{aligned}
\int_{\left(\mathbb{T}^{N}\right)^{2}} \rho_{\gamma}(x-y)|x-y| d x d y & \leq \gamma \\
\int_{\left(\mathbb{T}^{N}\right)^{2}} \gamma_{2}(\zeta, \xi) d v_{x, s}^{1, \varepsilon} \otimes d \bar{v}_{y, s}^{2, \varepsilon}(\xi, \zeta) & \leq 1
\end{aligned}
$$

it follows that

$$
\tilde{J}_{3,1} \leq \sqrt{D_{1}} \gamma(T+M)
$$

Using the same method as the estimate of $\tilde{K}_{2,2}$ in Theorem 4.3, we have

$$
\begin{aligned}
\tilde{J}_{4,1} \leq & 2 \sqrt{D_{1}} \delta(T+M) \\
& +\sqrt{D_{1}} \int_{0}^{t}\left|h^{\varepsilon}(s)\right|_{U} \int_{\left(\mathbb{T}^{N}\right)^{2}} \int_{\mathbb{R}^{2}} \rho_{\gamma}(x-y) \psi_{\delta}(\xi-\zeta)\left(f_{1}^{ \pm} \bar{f}_{2}^{ \pm}+\bar{f}_{1}^{ \pm} f_{2}^{ \pm}\right) d \xi d \zeta d x d y d s
\end{aligned}
$$

Hence,

$$
\begin{aligned}
\tilde{J}_{3}+\tilde{J}_{4}= & \tilde{J}_{3}+\tilde{J}_{4} \\
\leq & \sqrt{D_{1}}(\gamma+2 \delta)(T+M) \\
& +\sqrt{D_{1}} \int_{0}^{t}\left|h^{\varepsilon}(s)\right|_{U} \int_{\left(\mathbb{T}^{N}\right)^{2}} \int_{\mathbb{R}^{2}} \rho_{\gamma}(x-y) \psi_{\delta}(\xi-\zeta)\left(f_{1}^{ \pm} \bar{f}_{2}^{ \pm}+\bar{f}_{1}^{ \pm} f_{2}^{ \pm}\right) d \xi d \zeta d x d y d s .
\end{aligned}
$$


Combing all the previous estimates, it follows that

$$
\begin{aligned}
& \int_{\left(\mathbb{T}^{N}\right)^{2}} \int_{\mathbb{R}^{2}} \rho_{\gamma}(x-y) \psi_{\delta}(\xi-\zeta)\left(f_{1}^{ \pm}(x, t, \xi) \bar{f}_{2}^{ \pm}(y, t, \zeta)+\bar{f}_{1}^{ \pm}(x, t, \xi) f_{2}^{ \pm}(y, t, \zeta)\right) d \xi d \zeta d x d y \\
\leq & \int_{\mathbb{T}^{N}} \int_{\mathbb{R}}\left(f_{1,0}(x, \xi) \bar{f}_{2,0}(x, \xi)+\bar{f}_{1,0}(x, \xi) f_{2,0}(x, \xi)\right) d x d \xi+\mathcal{E}_{0}(\gamma, \delta)+2 T C_{p} \delta \gamma^{-1} \\
& +2 \varepsilon C D_{0} T \delta^{-1}+2 \sqrt{D_{1}}(2 \delta+\gamma)(T+M)+\left|\tilde{J}_{7}\right|(t)+\left|\tilde{\bar{J}}_{7}\right|(t) \\
& +2 \sqrt{D_{1}} \int_{0}^{t}\left|h^{\varepsilon}(s)\right|_{U} \int_{\left(\mathbb{T}^{N}\right)^{2}} \int_{\mathbb{R}^{2}} \rho_{\gamma}(x-y) \psi_{\delta}(\xi-\zeta)\left(f_{1}^{ \pm} \bar{f}_{2}^{ \pm}+\bar{f}_{1}^{ \pm} f_{2}^{ \pm}\right) d \xi d \zeta d x d y d s .
\end{aligned}
$$

Applying Gronwall inequality, we get

$$
\begin{aligned}
& \int_{\left(\mathbb{T}^{N}\right)^{2}} \int_{\mathbb{R}^{2}} \rho_{\gamma}(x-y) \psi_{\delta}(\xi-\zeta)\left(f_{1}^{ \pm}(x, t, \xi) \bar{f}_{2}^{ \pm}(y, t, \zeta)+\bar{f}_{1}^{ \pm}(x, t, \xi) f_{2}^{ \pm}(y, t, \zeta)\right) d \xi d \zeta d x d y \\
\leq & e^{2 \sqrt{D_{1}}(T+M)}\left[\int_{\mathbb{T}^{N}} \int_{\mathbb{R}}\left(f_{1,0} \bar{f}_{2,0}+\bar{f}_{1,0} f_{2,0}\right) d x d \xi+\mathcal{E}_{0}(\gamma, \delta)\right] \\
& +e^{2 \sqrt{D_{1}}(T+M)}\left[2 T C_{p} \delta \gamma^{-1}+2 \varepsilon C D_{0} T \delta^{-1}+2 \sqrt{D_{1}}(2 \delta+\gamma)(T+M)+\left|\tilde{J}_{7}\right|(t)+\left|\tilde{\bar{J}}_{7}\right|(t)\right] .
\end{aligned}
$$

Thus, collecting all the above estimates, we deduce that

$$
\begin{aligned}
& \int_{\mathbb{T}^{N}} \int_{\mathbb{R}}\left(f_{1}^{ \pm}(x, t, \xi) \bar{f}_{2}^{ \pm}(x, t, \xi)+\bar{f}_{1}^{ \pm}(x, t, \xi) f_{2}^{ \pm}(x, t, \xi)\right) d x d \xi \\
= & \int_{\left(\mathbb{T}^{N}\right)^{2}} \int_{\mathbb{R}^{2}}\left(f_{1}^{ \pm}(x, t, \xi) \bar{f}_{2}^{ \pm}(y, t, \zeta)+\bar{f}_{1}^{ \pm}(x, t, \xi) f_{2}^{ \pm}(y, t, \zeta)\right) \rho_{\gamma}(x-y) \psi_{\delta}(\xi-\zeta) d x d y d \xi d \zeta+\mathcal{E}_{t}(\gamma, \delta) \\
\leq & e^{2 \sqrt{D_{1}}(T+M)}\left[\int_{\mathbb{T}^{N}} \int_{\mathbb{R}}\left(f_{1,0} \bar{f}_{2,0}+\bar{f}_{1,0} f_{2,0}\right) d x d \xi+\mathcal{E}_{0}(\gamma, \delta)\right] \\
& +e^{2 \sqrt{D_{1}}(T+M)}\left[2 T C_{p} \delta \gamma^{-1}+2 \varepsilon C D_{0} T \delta^{-1}+2 \sqrt{D_{1}}(2 \delta+\gamma)(T+M)+\left|\tilde{J}_{7}\right|(t)+\left|\tilde{\bar{J}}_{7}\right|(t)\right]+\mathcal{E}_{t}(\gamma, \delta) \\
:= & e^{2 \sqrt{D_{1}}(T+M)} \int_{\mathbb{T}^{N}} \int_{\mathbb{R}}\left(f_{1,0} \bar{f}_{2,0}+\bar{f}_{1,0} f_{2,0}\right) d x d \xi+e^{2 \sqrt{D_{1}}(T+M)}\left(\left|\tilde{J}_{7}\right|(t)+\left|\tilde{\bar{J}}_{7}\right|(t)\right)+r(\varepsilon, \gamma, \delta, t) .
\end{aligned}
$$

where the remainder is given by

$$
r(\varepsilon, \gamma, \delta, t)=e^{2 \sqrt{D_{1}}(T+M)}\left[2 T C_{p} \delta \gamma^{-1}+2 \varepsilon C D_{0} T \delta^{-1}+2 \sqrt{D_{1}}(2 \delta+\gamma)(T+M)+\mathcal{E}_{0}(\gamma, \delta)\right]+\mathcal{E}_{t}(\gamma, \delta) .
$$

Applying the Burkholder-Davis-Gundy inequality, and utilizing (5.68), (2.12) that

$$
\begin{aligned}
\mathbb{E} \sup _{t \in[0, T]}\left|\tilde{J}_{7}\right|(t) & \leq \sqrt{\varepsilon} \mathbb{E} \sup _{t \in[0, T]}\left|\sum_{k \geq 1} \int_{0}^{t} \int_{\left(\mathbb{T}^{N}\right)^{2}} \int_{\mathbb{R}^{2}} \bar{f}_{2}^{ \pm}(s, y, \zeta) g_{k}(x, \xi) \alpha d \zeta d v_{x, s}^{1, \varepsilon}(\xi) d x d y d \beta_{k}(s)\right| \\
& =\sqrt{\varepsilon} \mathbb{E} \sup _{t \in[0, T]}\left|\sum_{k \geq 1} \int_{0}^{t} \int_{\left(\mathbb{T}^{N}\right)^{2}} \int_{\mathbb{R}^{2}} \bar{f}_{2}^{ \pm}(s, y, \zeta) \partial_{\zeta} \gamma_{2}(\xi, \zeta) \rho_{\gamma}(x-y) g_{k}(x, \xi) d \zeta d v_{x, s}^{1, \varepsilon}(\xi) d x d y d \beta_{k}(s)\right| \\
& =\sqrt{\varepsilon} \mathbb{E} \sup _{t \in[0, T]}\left|\sum_{k \geq 1} \int_{0}^{t} \int_{\left(\mathbb{T}^{N}\right)^{2}} \int_{\mathbb{R}^{2}} \gamma_{2}(\xi, \zeta) \rho_{\gamma}(x-y) g_{k}(x, \xi) d v_{x, s}^{1, \varepsilon} \otimes d \bar{v}_{y, s}^{2, \varepsilon}(\xi, \zeta) d x d y d \beta_{k}(s)\right| \\
& \leq \sqrt{\varepsilon} \mathbb{E}\left[\int_{0}^{T} \int_{\left(\mathbb{T}^{N}\right)^{2}} \int_{\mathbb{R}^{2}} \gamma_{2}^{2}(\xi, \zeta) \rho_{\gamma}^{2}(x-y)\left(\sum_{k \geq 1} g_{k}^{2}(x, \xi)\right) d v_{x, s}^{1, \varepsilon} \otimes d \bar{v}_{y, s}^{2, \varepsilon}(\xi, \zeta) d x d y d s\right]^{\frac{1}{2}} \\
& \leq \sqrt{\varepsilon} \sqrt{D_{0}} \mathbb{E}\left[\int_{0}^{T} \int_{\left(\mathbb{T}^{N}\right)^{2}} \int_{\mathbb{R}^{2}} \gamma_{2}^{2}(\xi, \zeta) \rho_{\gamma}^{2}(x-y)\left(1+|\xi|^{2}\right) d v_{x, s}^{1, \varepsilon} \otimes d \bar{v}_{y, s}^{2, \varepsilon}(\xi, \zeta) d x d y d s\right]^{\frac{1}{2}} .
\end{aligned}
$$


Taking into account the following facts

$$
\begin{aligned}
& \int_{\mathbb{R}^{2}} \gamma_{2}^{2}(\xi, \zeta)\left(1+|\xi|^{2}\right) d v_{x, s}^{1, \varepsilon} \otimes d \bar{v}_{y, s}^{2, \varepsilon}(\xi, \zeta) \\
\leq & \int_{\mathbb{R}^{2}}\left(1+|\xi|^{2}\right) d v_{x, s}^{1, \varepsilon} \otimes d \bar{v}_{y, s}^{2, \varepsilon}(\xi, \zeta) \leq C
\end{aligned}
$$

and

$$
\int_{\left(\mathbb{T}^{N}\right)^{2}} \rho_{\gamma}^{2}(x-y) d x d y \leq \gamma^{-2 N}
$$

we further deduce that

$$
\mathbb{E} \sup _{t \in[0, T]}\left|\tilde{J}_{7}\right|(t) \leq C \sqrt{\varepsilon} \sqrt{D_{0} T} \gamma^{-N} .
$$

By the same method as above, we deduce that

$$
\mathbb{E} \sup _{t \in[0, T]}\left|\tilde{\overline{J_{7}}}\right|(t) \leq C \sqrt{\varepsilon} \sqrt{\overline{D_{0} T}} \gamma^{-N} .
$$

For the remainder, we have

$$
\begin{aligned}
\sup _{t \in[0, T]} r(\varepsilon, \gamma, \delta, t) \leq & 2 e^{2 \sqrt{D_{1}}(T+M)}\left[T C_{p} \delta \gamma^{-1}+\varepsilon C D_{0} T \delta^{-1}+\sqrt{D_{1}}(2 \delta+\gamma)(T+M)\right] \\
& +\left(e^{2 \sqrt{D_{1}}(T+M)}+1\right) \sup _{t \in[0, T]} \mathcal{E}_{t}(\gamma, \delta) .
\end{aligned}
$$

In the following, we aim to prove the error term $\sup _{t \in[0, T]} \mathcal{E}_{t}(\gamma, \delta) \rightarrow 0$ as $\gamma, \delta \rightarrow 0$. To achieve it, we adopt similar method as the proof of Proposition 6.1 and Theorem 6.2 in [8].

For any $t \in[0, T]$, we have

$$
\begin{aligned}
\mathcal{E}_{t}(\gamma, \delta)= & \int_{\mathbb{T}^{N}} \int_{\mathbb{R}}\left(f_{1}^{ \pm}(x, t, \xi) \bar{f}_{2}^{ \pm}(x, t, \xi)+\bar{f}_{1}^{ \pm}(x, t, \xi) f_{2}^{ \pm}(x, t, \xi)\right) d \xi d x \\
& -\int_{\left.\mathbb{T}^{N}\right)^{2}} \int_{\mathbb{R}^{2}}\left(f_{1}^{ \pm}(x, t, \xi) \bar{f}_{2}^{ \pm}(y, t, \zeta)+\bar{f}_{1}^{ \pm}(x, t, \xi) f_{2}^{ \pm}(y, t, \zeta)\right) \rho_{\gamma}(x-y) \psi_{\delta}(\xi-\zeta) d x d y d \xi d \zeta \\
= & {\left[\int_{\mathbb{T}^{N}} \int_{\mathbb{R}}\left(f_{1}^{ \pm}(x, t, \xi) \bar{f}_{2}^{ \pm}(x, t, \xi)+\bar{f}_{1}^{ \pm}(x, t, \xi) f_{2}^{ \pm}(x, t, \xi)\right) d \xi d x\right.} \\
& \left.-\int_{\left(\mathbb{T}^{N}\right)^{2}} \int_{\mathbb{R}} \rho_{\gamma}(x-y)\left(f_{1}^{ \pm}(x, t, \xi) \bar{f}_{2}^{ \pm}(y, t, \xi)+\bar{f}_{1}^{ \pm}(x, t, \xi) f_{2}^{ \pm}(y, t, \xi)\right) d \xi d x d y\right] \\
& +\left[\int_{\left(\mathbb{T}^{N}\right)^{2}} \int_{\mathbb{R}} \rho_{\gamma}(x-y)\left(f_{1}^{ \pm}(x, t, \xi) \bar{f}_{2}^{ \pm}(y, t, \xi)+\bar{f}_{1}^{ \pm}(x, t, \xi) f_{2}^{ \pm}(y, t, \xi)\right) d \xi d x d y\right. \\
& \left.-\int_{\left(\mathbb{T}^{N}\right)^{2}} \int_{\mathbb{R}^{2}}\left(f_{1}^{ \pm}(x, t, \xi) \bar{f}_{2}^{ \pm}(y, t, \zeta)+\bar{f}_{1}^{ \pm}(x, t, \xi) f_{2}^{ \pm}(y, t, \zeta)\right) \rho_{\gamma}(x-y) \psi_{\delta}(\xi-\zeta) d x d y d \xi d \zeta\right] \\
:= & H_{1}+H_{2},
\end{aligned}
$$

Applying the same method as (4.34) and (4.35), it follows that

$$
\left|H_{2}\right| \leq 2 \delta
$$


Moreover, it is easy to deduce that

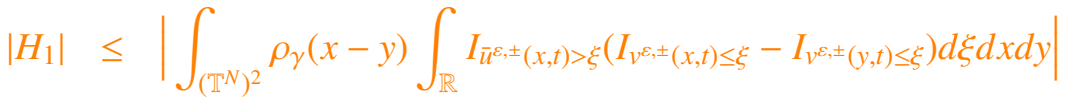

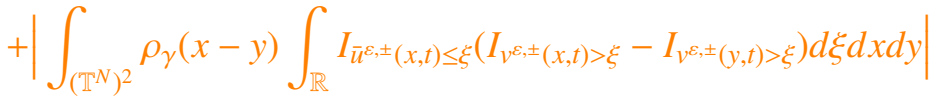

$$
\begin{aligned}
& \leq 2 \int_{\left(\mathbb{T}^{N}\right)^{2}} \rho_{\gamma}(x-y)\left|v^{\varepsilon, \pm}(x, t)-v^{\varepsilon, \pm}(y, t)\right| d x d y .
\end{aligned}
$$

By (5.71) and (4.38), we have

$$
\begin{aligned}
& \int_{\left(\mathbb{T}^{N}\right)^{2}} \rho_{\gamma}(x-y)\left|v^{\varepsilon, \pm}(x, t)-v^{\varepsilon, \pm}(y, t)\right| d x d y \\
= & \int_{\left(\mathbb{T}^{N}\right)^{2}} \int_{\mathbb{R}} \rho_{\gamma}(x-y)\left(f_{2}^{ \pm}(x, t, \xi) \bar{f}_{2}^{ \pm}(y, t, \xi)+\bar{f}_{2}^{ \pm}(x, t, \xi) f_{2}^{ \pm}(y, t, \xi)\right) d \xi d x d y \\
\leq & \int_{\left(\mathbb{T}^{N}\right)^{2}} \int_{\mathbb{R}^{2}} \rho_{\gamma}(x-y) \psi_{\delta}(\xi-\zeta)\left(f_{2}^{ \pm}(x, t, \xi) \bar{f}_{2}^{ \pm}(y, t, \zeta)+\bar{f}_{2}^{ \pm}(x, t, \xi) f_{2}^{ \pm}(y, t, \zeta)\right) d \xi d \zeta d x d y+2 \delta \\
\leq & e^{2 \sqrt{D_{1}}(T+M)}\left[\int_{\mathbb{T}^{N}} \int_{\mathbb{R}}\left(f_{2,0} \bar{f}_{2,0}+\bar{f}_{2,0} f_{2,0}\right) d \xi d x+\mathcal{E}_{0}(\gamma, \delta)\right] \\
& +2 e^{2 \sqrt{D_{1}}(T+M)}\left[T C_{p} \delta \gamma^{-1}+\sqrt{D_{1}}(\gamma+2 \delta)(T+M)\right]+2 \delta \\
= & e^{2 \sqrt{D_{1}}(T+M)} \mathcal{E}_{0}(\gamma, \delta)+2 e^{2 \sqrt{D_{1}}(T+M)}\left[T C_{p} \delta \gamma^{-1}+\sqrt{D_{1}}(\gamma+2 \delta)(T+M)\right]+2 \delta,
\end{aligned}
$$

where $\mathcal{E}_{0}(\gamma, \delta) \rightarrow 0$, when $\gamma, \delta \rightarrow 0$. Then,

$$
\left|H_{1}\right| \leq 4 \delta+2 e^{2 \sqrt{D_{1}}(T+M)} \mathcal{E}_{0}(\gamma, \delta)+4 e^{2 \sqrt{D_{1}}(T+M)}\left[T C_{p} \delta \gamma^{-1}+\sqrt{D_{1}}(\gamma+2 \delta)(T+M)\right] .
$$

Combing all the above estimates, we conclude that

$$
\sup _{t \in[0, T]} \mathcal{E}_{t}(\gamma, \delta) \leq 6 \delta+2 e^{2 \sqrt{D_{1}}(T+M)} \mathcal{E}_{0}(\gamma, \delta)+4 e^{2 \sqrt{D_{1}}(T+M)}\left[T C_{p} \delta \gamma^{-1}+\sqrt{D_{1}}(\gamma+2 \delta)(T+M)\right] .
$$

Hence, we deduce from (5.70) that

$$
\begin{aligned}
& \sup _{t \in[0, T]} r(\varepsilon, \gamma, \delta, t) \\
\leq \quad & 2 e^{2 \sqrt{D_{1}}(T+M)}\left[T C_{p} \delta \gamma^{-1}+\varepsilon C D_{0} T \delta^{-1}+\sqrt{D_{1}}(2 \delta+\gamma)(T+M)\right] \\
& +6\left(e^{2 \sqrt{D_{1}}(T+M)}+1\right) \delta+2 e^{2 \sqrt{D_{1}}(T+M)}\left(e^{\sqrt{D_{1}}(T+M)}+1\right) \mathcal{E}_{0}(\gamma, \delta) \\
+ & 4 e^{2 \sqrt{D_{1}}(T+M)}\left(e^{\sqrt{D_{1}}(T+M)}+1\right)\left[T C_{p} \delta \gamma^{-1}+\sqrt{D_{1}}(\gamma+2 \delta)(T+M)\right] .
\end{aligned}
$$

\section{Letting}

$$
\delta=\gamma^{\frac{4}{3}}, \quad \gamma=\varepsilon^{\frac{1}{2(1+N)}},
$$

then,

$$
\mathbb{E} \sup _{t \in[0, T]}\left|\tilde{J}_{7}\right|(t) \leq C \sqrt{D_{0} T} \varepsilon^{\frac{1}{2(1+N)}} \rightarrow 0 \quad \varepsilon \rightarrow 0
$$


and

$$
\mathbb{E} \sup _{t \in[0, T]}\left|\tilde{\bar{J}}_{7}\right|(t) \leq C \sqrt{D_{0} T} \varepsilon^{\frac{1}{2(1+N)}} \rightarrow 0 \quad \varepsilon \rightarrow 0,
$$

which implies that $\sup _{t \in[0, T]}\left|\tilde{J}_{7}\right|(t) \rightarrow 0$ in probability and $\sup _{t \in[0, T]}\left|\tilde{\bar{J}}_{7}\right|(t) \rightarrow 0$ in probability, as $\varepsilon \rightarrow 0$ by Chebyshev inequality. Moreover, it follows that

$$
\begin{array}{ll} 
& \sup _{t \in[0, T]} r(\varepsilon, \gamma, \delta, t) \\
\leq \quad & 2 e^{2 \sqrt{D_{1}}(T+M)}\left[T C_{p} \varepsilon^{\frac{1}{6(1+N)}}+C D_{0} T \varepsilon^{\frac{1+3 N}{3(1+N)}}+\sqrt{D_{1}}\left(2 \varepsilon^{\frac{2}{3(1+N)}}+\varepsilon^{\frac{1}{2(1+N)}}\right)(T+M)\right] \\
& +6\left(e^{2 \sqrt{D_{1}}(T+M)}+1\right) \varepsilon^{\frac{2}{3(1+N)}}+2 e^{2 \sqrt{D_{1}}(T+M)}\left(e^{2 \sqrt{D_{1}}(T+M)}+1\right) \mathcal{E}_{0}(\gamma, \delta) \\
& +4 e^{2 \sqrt{D_{1}}(T+M)}\left(e^{2 \sqrt{D_{1}}(T+M)}+1\right)\left[T C_{p} \varepsilon^{\frac{1}{6(1+N)}}+\sqrt{D_{1}}\left(2 \varepsilon^{\frac{2}{3(1+N)}}+\varepsilon^{\frac{1}{2(1+N)}}\right)(T+M)\right] \\
\rightarrow \quad 0, \quad \text { as } \quad \varepsilon \rightarrow 0 .
\end{array}
$$

Notice that $f_{1}=I_{\bar{u}^{\varepsilon}>\xi}$ and $f_{2}=I_{v^{\varepsilon}>\xi}$ with initial data $f_{1,0}=I_{u_{0}>\xi}$ and $f_{2,0}=I_{u_{0}>\xi}$, respectively. With the help of identity (4.41), we deduce from (5.69) that

$$
\left\|\bar{u}^{\varepsilon}(t)-v^{\varepsilon}(t)\right\|_{L^{1}\left(\mathbb{T}^{N}\right)} \leq e^{2 \sqrt{D_{1}}(T+M)}\left(\left|\tilde{J}_{7}\right|(t)+\left|\tilde{\bar{J}}_{7}\right|(t)\right)+r(\varepsilon, \gamma, \delta, t) .
$$

Hence, it follows from (5.72) that

$$
\begin{aligned}
& \left\|\bar{u}^{\varepsilon}-v^{\varepsilon}\right\|_{L^{1}\left([0, T] ; L^{1}\left(\mathbb{T}^{N}\right)\right)} \\
\leq & T \cdot e s s \sup _{t \in[0, T]}\left\|\bar{u}^{\varepsilon}(t)-v^{\varepsilon}(t)\right\|_{L^{1}\left(\mathbb{T}^{N}\right)} \\
\leq & T e^{2 \sqrt{D_{1}}(T+M)}\left(\sup _{t \in[0, T]}\left|\tilde{J}_{7}\right|(t)+\sup _{t \in[0, T]}\left|\tilde{\bar{J}}_{7}\right|(t)\right)+T \cdot \sup _{t \in[0, T]} r(\varepsilon, \gamma, \delta, t) \rightarrow 0
\end{aligned}
$$

in probability as $\varepsilon \rightarrow 0$. We complete the proof.

Acknowledgements The authors are grateful to the anonymous referees for their constructive comments and valuable suggestions. This work is partly supported by National Natural Science Foundation of China (No. 11801032). Key Laboratory of Random Complex Structures and Data Science, Academy of Mathematics and Systems Science, Chinese Academy of Sciences (No. 2008DP173182). China Postdoctoral Science Foundation funded project (No. 2018M641204).

\section{References}

[1] K. Ammar, P. Willbold, J. Carrillo: Scalar conservation laws with general boundary condition and continuous flux function. J. Differential Equations 228, no. 1, 111-139 (2006).

[2] M. Boué, P. Dupuis: A variational representation for certain functionals of Brownian motion. Ann. Probab. 26 (4) 1641-1659 (1998). 
[3] A. Budhiraja, P. Dupuis: A variational representation for positive functionals of infinite dimensional Brownian motion. Probab. Math. Statist. 20, 39-61 (2000).

[4] F. Bouchut, L. Desvillettes: Averaging lemmas without time Fourier transform and application to discretized kinetic equations. Proc. Roy. Soc. Edinburgh Sect. A 129, no. 1, 19-36 (1999).

[5] G. Chen, H. Frid: Decay of entropy solutions of nonlinear conservation laws. Arch. Ration. Mech. Anal. 146, no. 2, 95-127 (1999).

[6] C.M. Dafermos: Hyperbolic Conservation Laws in Continuum Physics. 2nd edn. Berlin, Springer (2005).

[7] C. De Lellis, M. Westdickenberg: On the optimality of velocity averaging lemmas. Ann. Inst. H. Poincaré Anal. Non Linéaire 20, no. 6, 1075-1085 (2003).

[8] A. Debussche, M. Hofmanová, and J. Vovelle: Degenerate parabolic stochastic partial differential equations: Quasilinear case. Ann. Probab. 44, no. 3, 1916-1955 (2016).

[9] A. Debussche, J. Vovelle: Scalar conservation laws with stochastic forcing (revised version). http://math.univ-lyon1.fr/vovelle/DebusscheVovelleRevised. J. Funct. Anal. 259, no. 4, 1014-1042 (2010).

[10] A. Debussche, J. Vovelle: Invariant measure of scalar first-order conservation laws with stochastic forcing. Probab. Theory Related Fields 163, no. 3-4, 575-611 (2015).

[11] A. Dembo, O. Zeitouni: Large Deviations Techniques and Applications. Boston, Jones and Bartlett (1993).

[12] P. Dupuis, R.S. Ellis: A Weak Convergence Approach to the Theory of Large Deviations. New York, Wiley (1997).

[13] J. Feng, D. Nualart: Stochastic scalar conservation laws. J. Funct. Anal. 255, no. 2, 313-373 (2008).

[14] F. Flandoli, D. Gatarek: Martingale and stationary solutions for stochastic Navier-Stokes equations. Probab. Theory Related Fields 102, no. 3, 367-391 (1995).

[15] I. Gyöngy, C. Rovira : On $L^{p}$ solutions of semilinear stochastic partial differential equations. Stochastic Process. Appl.90, no. 1, 83-108 (2000).

[16] C. Imbert, J. Vovelle: A kinetic formulation for multidimensional scalar conservation laws with boundary conditions and applications. SIAM J. Math. Anal. 36, no. 1,214-232 (2004).

[17] J.U. Kim: On a stochastic scalar conservation law. Indiana Univ. Math. J. 52 227-256 (2003).

[18] K. Kobayasi, D. Noboriguchi: A stochastic conservation law with nonhomogeneous Dirichlet boundary conditions. Acta Math. Vietnam. 41, no. 4, 607-632 (2016). 
[19] P.L. Lions, B. Perthame, E. Tadmor: A kinetic formulation of multidimensional scalar conservation laws and related equations. J. of A.M.S., 7, 169-191 (1994).

[20] M. Mariani: Large deviation principles for stochastic scalar conservation laws. Probab. Theory Related Fields 147, 607-648 (2010).

[21] M. Mariani: Large Deviations for Stochastic Conservation Laws and Their Variational Counterparts. Ph D Thesis, Sapienza Universitá di Roma (2007).

[22] A. Matoussi, W. Sabbagh, T. Zhang: Large Deviation Principles of Obstacle Problems for Quasilinear Stochastic PDEs. arXiv:1712.02169.

[23] F. Otto: Initial-boundary value problem for a scalar conservation law. C. R. Acad. Sci. Paris Sér. I Math. 322, no. 8, 729-734 (1996).

[24] A. Porretta, J. Vovelle: $L^{1}$ solutions to first order hyperbolic equations in bounded domains. Comm. Partial Differential Equations 28, no. 1-2, 381-408 (2003).

[25] G. Vallet, P. Wittbold: On a stochastic first-order hyperbolic equation in a bounded domain. Infin. Dimens. Anal. Quantum Probab. Relat. Top. 12, no. 4, 613-651 (2009). 